\title{
Aerobic Oxidative C-H Olefination of Arylamides with Unactivated Olefins via a Rh(III)-Catalyzed C-H Activation
}

Subramanian Jambu, Chikkabagilu Nagaraju Shambhavi and Masilamani Jeganmohan*

Department of Chemistry, Indian Institute of Technology Madras, Chennai 600036,

Tamil Nadu, India

Email:mjeganmohan@iitm.ac.in

\section{Supporting Information (SI)}

Table of Contents
S2- S3
Experimental Section
S4 - S6
Mechanistic Studies (Deuterium labelling)
S7
Optimization Studies
S8 - S9
X-Ray analysis
S10
Reference
S11 - S32
Spectral Data of all Compounds
S33 - S75
Copies of ${ }^{1} \mathrm{H}$ and ${ }^{13} \mathrm{C}$ NMR Spectra of All Compounds. 


\section{Experimental Section}

General Information. All reactions were carried out under the $\mathrm{N}_{2}$ atmosphere in flame-dried glassware. Syringes which were used to transfer anhydrous solvents or reagents were purged with nitrogen prior to use (three times). Dry solvents were used for the reaction. Column chromatographical purifications were performed using $\mathrm{SiO}_{2}$ (100-200 mesh ASTM) from Avra Pvt. Ltd., India. Abbreviations for signal coupling are as follows: s, singlet; d, doublet; t, triplet; q, quartet; m, multiplet. Arylamides, $\left.{ }^{1}\left[\mathrm{Rh}\left(\mathrm{Cp}^{*}\right)\left(\mathrm{CH}_{3} \mathrm{CN}\right)_{3}\right)\right]\left[\mathrm{SbF}_{6}\right]_{2},{ }^{2}$ were prepared according to literature procedures. Commercially available Alkenes, metal salts and other chemicals were purchased from Sigma-Aldrich and Spectrochem. Pvt. Ltd., India. These chemicals were used without further purification.

\section{General Procedure for the Alkenylation Reaction.}

Arylamides 1 (50 mg) (1 equiv), $\left.\left[\mathrm{Rh}\left(\mathrm{Cp}^{*}\right)\left(\mathrm{CH}_{3} \mathrm{CN}\right)_{3}\right)\right]\left[\mathrm{SbF}_{6}\right]_{2} \quad(5 \mathrm{~mol} \%)$ and 1 adamantanecarboxylic acid (50 mol \%) were taken in a $15-\mathrm{mL}$ pressure tube. Dry tetrahydrofuran (THF) (2.0 mL) was added to the reaction mixture. Then, alkenes 2 (3.0 equiv) was added followed by the addition of dry THF $(1.0 \mathrm{~mL})$ and the reaction mixture was stirred at open air (under air atmosphere) for five minutes. Then, a screw cap was used to cover the tube and the reaction mixture was allowed to stir at $110{ }^{\circ} \mathrm{C}$ for $24 \mathrm{~h}$. Then, the reaction mixture was diluted with $\mathrm{CH}_{2} \mathrm{Cl}_{2}$, filtered through celite, and the filtrate was concentrated. The crude residue was purified by column chromatography silica-gel 120-200 mesh, EtOAc:Hexane gave alkenylated product $\mathbf{3}$. In the case of functionalized unactivated alkenes $\mathbf{2 h}-\mathbf{l}, 2.0$ equiv was used.

\section{Procedure for the alkenylation of 4-Methoxy benzamide (1a) with 1-hexene (2a) Catalyzed by a Rhodium Complex (1 mmol scale).}

4-Methoxy benzamide 1a $\left.(205 \mathrm{mg}, 1 \mathrm{mmol}),\left[\mathrm{Rh}\left(\mathrm{Cp}^{*}\right)\left(\mathrm{CH}_{3} \mathrm{CN}\right)_{3}\right)\right]\left[\mathrm{SbF}_{6}\right]_{2}(5 \mathrm{~mol} \%, 0.05$ $\mathrm{mmol}, 41.5 \mathrm{mg}$ ) and 1-adamantanecarboxylic acid (50 mol \%, $0.5 \mathrm{mmol}, 90.4 \mathrm{mg}$ ) were taken in a $15-\mathrm{mL}$ pressure tube. Dry tetrahydrofuran (THF) $(3.0 \mathrm{~mL})$ was added to the reaction mixture. Then, 1-hexene $\mathbf{2 a}$ ( 3.0 equiv, $3 \mathrm{mmol}$ ) was added followed by the addition of dry THF (3.0 mL) and the reaction mixture was stirred at open air (under air atmosphere) for five minutes. Then, a screw cap was used to cover the tube and the reaction mixture was allowed to stir at $110{ }^{\circ} \mathrm{C}$ for $24 \mathrm{~h}$. Then, the reaction mixture was diluted with $\mathrm{CH}_{2} \mathrm{Cl}_{2}$, filtered through celite, and the filtrate was concentrated. The crude residue was purified by column 
chromatography silica-gel 120-200 mesh, EtOAc:Hexane gave alkenylated product 3aa. 200 $\mathrm{mg}$ in $70 \%$ yield as pale-yellow liquid.

\section{Preparation of Starting Materials 1 and 2.}

Substituted arylamides $\mathbf{1} \mathbf{a}-\mathbf{v}$ and coumarin, saccharine and phthalimide containing unactivated olefines were prepared by the known reported procedure. ${ }^{1,3}$ Remaining unactivated olefines are purchased from Sigma-Aldrich and Spectrochem. Pvt. Ltd., India. 


\section{Mechanistic Investigation}

\section{Deuterium Labelling Studies for D-1a.}

A $15 \mathrm{~mL}$ pressure tube with a septum containing arylamide 1a $(75 \mathrm{mg})$ and $\left[\left[\mathrm{Rh}\left(\mathrm{Cp}^{*}\right)\left(\mathrm{CH}_{3} \mathrm{CN}\right)_{3}\right)\right]\left[\mathrm{SbF}_{6}\right]_{2}(5 \mathrm{~mol} \%)$, dry $\mathrm{THF}(2.0 \mathrm{~mL})$ were added to the reaction. Then, $\mathrm{CD}_{3} \mathrm{COOD}$ (2.0 equiv) and dry THF $(1.0 \mathrm{~mL})$ were added via and the reaction mixture was stirred at open air (under air atmosphere) for five minutes. Then, a screw cap was used to cover the tube and the reaction mixture was allowed to stir at $110^{\circ} \mathrm{C}$ for $6 \mathrm{~h}$. Then, the reaction mixture was diluted with $\mathrm{CH}_{2} \mathrm{Cl}_{2}$, filtered through Celite, and the filtrate was concentrated. The crude residue was purified by column chromatography silica-gel 120-200 mesh, EtOAc:Hexane gave product D-1a. In the reaction, product D-1a was observed in $96 \%$ yield with $61 \%$ of deuterium incorporation at the both ortho carbons of benamide. This result clearly reveals that the $\mathrm{C}-\mathrm{H}$ bond activation as a key intermediate in the reaction as well as it is the reversible process.

\section{Preparation of Compounds D-3aa.}

Arylamide 1a (75 mg) (1.0 equiv) and $\left.\left[\mathrm{Rh}\left(\mathrm{Cp}^{*}\right)\left(\mathrm{CH}_{3} \mathrm{CN}\right)_{3}\right)\right]\left[\mathrm{SbF}_{6}\right]_{2}(5 \mathrm{~mol} \%)$, dry THF $(2.0$ $\mathrm{mL}$ ) were taken in a $15-\mathrm{mL}$ pressure tube. $\mathrm{CD}_{3} \mathrm{COOD}$ (2.0 equiv) were added to the reaction mixture. Then, alkene $\mathbf{2 b}$ (3.0 equiv) was added followed by the addition of dry THF (1.0 mL) and the reaction mixture was stirred at open air (under air atmosphere) for five minutes. Then, a screw cap was used to cover the tube and the reaction mixture was allowed to stir at $110^{\circ} \mathrm{C}$ for $6 \mathrm{~h}$. Then, the reaction mixture was diluted with $\mathrm{CH}_{2} \mathrm{Cl}_{2}$, filtered through Celite, and the filtrate was concentrated. The crude residue was purified by column chromatography silica-gel 120-200 mesh, EtOAc:Hexane gave product D-3aa in 58\%. In the reaction, 65\% of deuterium incorporation was observed at the ortho carbon of alkenylated benzamide. This result also clearly reveals that the $\mathrm{C}-\mathrm{H}$ bond activation as a key intermediate in the reaction as well as it is the reversible process. 


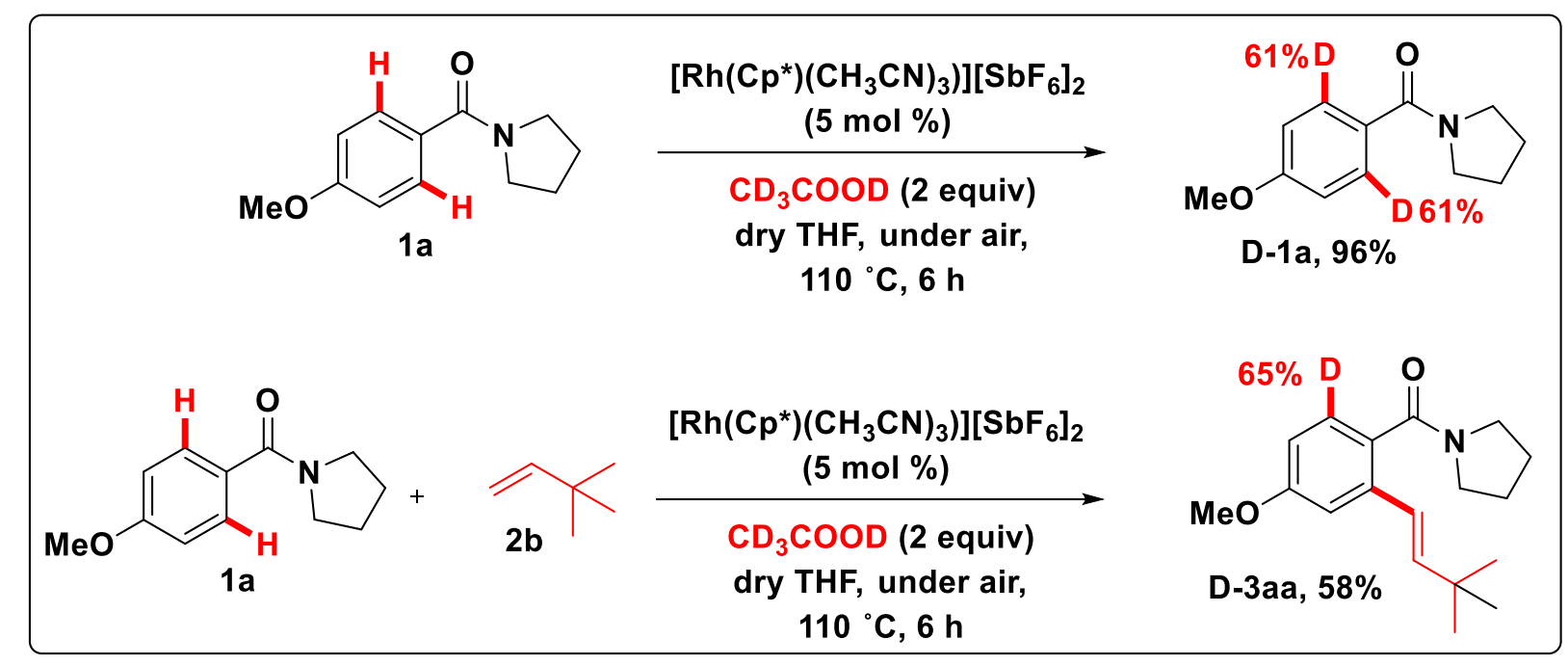


${ }^{1} \mathrm{H}$ Spectra of Compound D-1a.

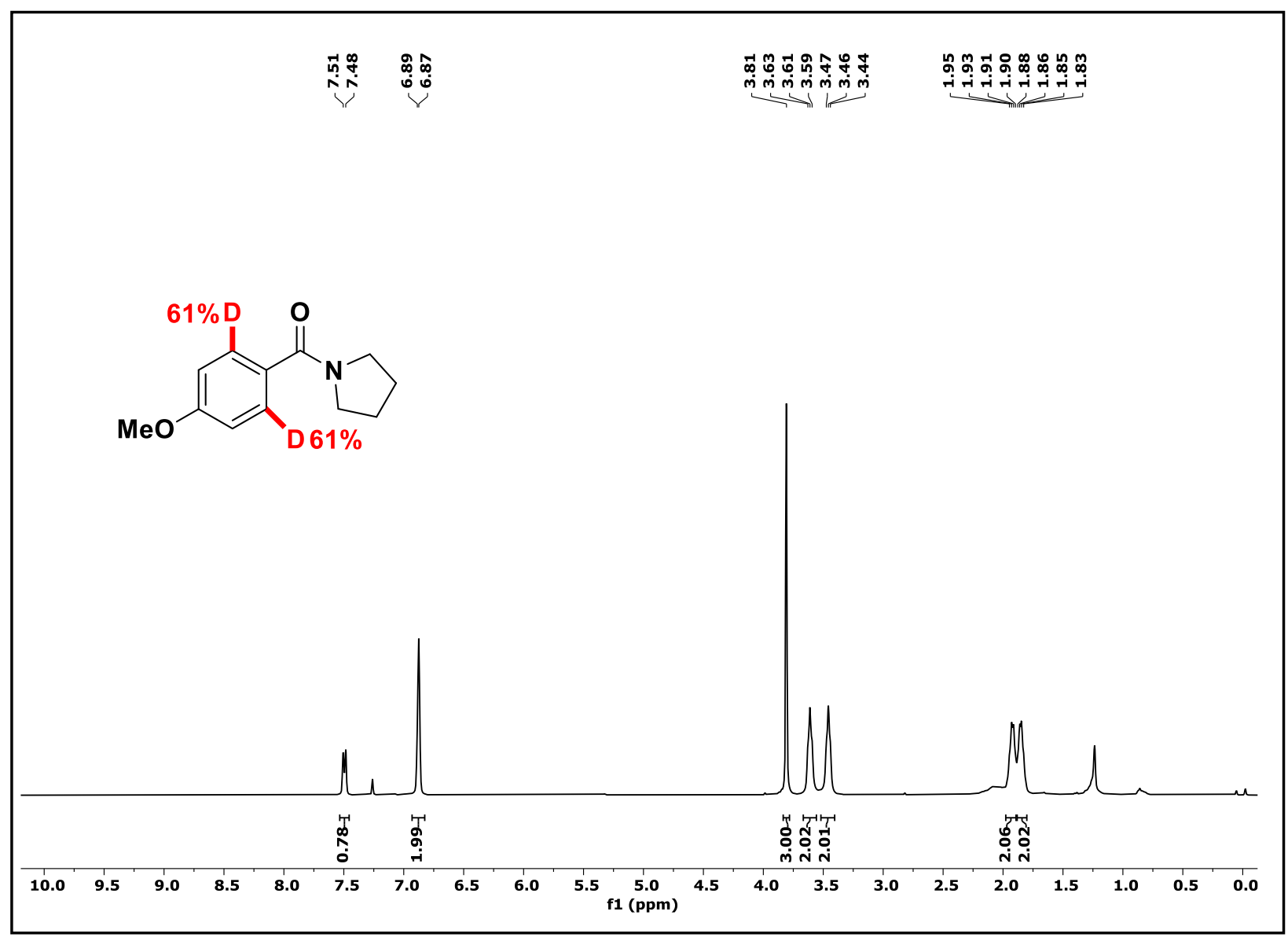

${ }^{1} \mathrm{H}$ Spectra of Compound D-3aa.

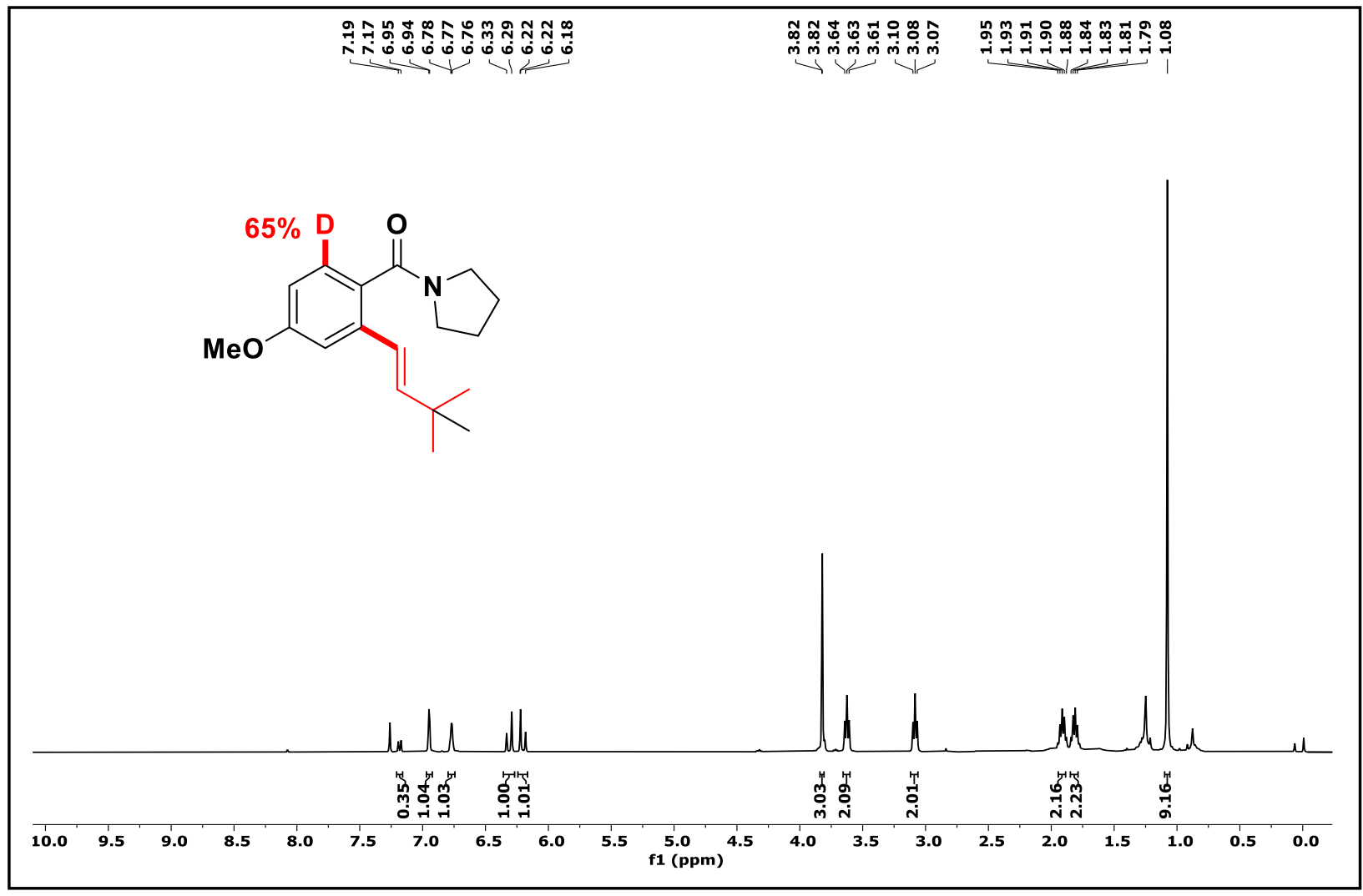


Table S1. Optimization of Olefination Reaction ${ }^{a}$

\begin{tabular}{|c|c|c|c|c|c|}
\hline S. No & Solvent & Catalyst & Additive & temperature & Yield $(\%)^{\mathrm{b}}$ \\
\hline 1 & THF & $\left.\left[\mathrm{Rh}\left(\mathrm{Cp}^{*}\right)\left(\mathrm{CH}_{3} \mathrm{CN}\right)_{3}\right)\right]\left[\mathrm{SbF}_{6}\right]_{2}$ & $\mathrm{AcOH}$ & $110^{\circ} \mathrm{C}$ & 65 \\
\hline 2 & THF & $\left.\left[\mathrm{Rh}\left(\mathrm{Cp}^{*}\right)\left(\mathrm{CH}_{3} \mathrm{CN}\right)_{3}\right)\right]\left[\mathrm{SbF}_{6}\right]_{2}$ & $\begin{array}{l}\text { Mesitylene } \\
\text { carboxylic } \\
\text { acid }\end{array}$ & $110^{\circ} \mathrm{C}$ & 53 \\
\hline 3 & THF & $\left.\left[\mathrm{Rh}\left(\mathrm{Cp}^{*}\right)\left(\mathrm{CH}_{3} \mathrm{CN}\right)_{3}\right)\right]\left[\mathrm{SbF}_{6}\right]_{2}$ & Ad-1-COOH & $110{ }^{\circ} \mathrm{C}$ & 72 \\
\hline 4 & THF & $\left.\left[\mathrm{Rh}\left(\mathrm{Cp}^{*}\right)\left(\mathrm{CH}_{3} \mathrm{CN}\right)_{3}\right)\right]\left[\mathrm{SbF}_{6}\right]_{2}$ & $\mathrm{PivOH}$ & $110^{\circ} \mathrm{C}$ & 55 \\
\hline 5 & THF & - & Ad-1-COOH & $110^{\circ} \mathrm{C}$ & NR \\
\hline 6 & THF & $\left.\left[\mathrm{Rh}\left(\mathrm{Cp}^{*}\right)\left(\mathrm{CH}_{3} \mathrm{CN}\right)_{3}\right)\right]\left[\mathrm{SbF}_{6}\right]_{2}$ & - & $110^{\circ} \mathrm{C}$ & 45 \\
\hline 7 & DMF & $\left.\left[\mathrm{Rh}\left(\mathrm{Cp}^{*}\right)\left(\mathrm{CH}_{3} \mathrm{CN}\right)_{3}\right)\right]\left[\mathrm{SbF}_{6}\right]_{2}$ & $\mathrm{Ad}-1-\mathrm{COOH}$ & $110^{\circ} \mathrm{C}$ & trace \\
\hline 8 & toluene & $\left.\left[\mathrm{Rh}\left(\mathrm{Cp}^{*}\right)\left(\mathrm{CH}_{3} \mathrm{CN}\right)_{3}\right)\right]\left[\mathrm{SbF}_{6}\right]_{2}$ & Ad-1-COOH & $110^{\circ} \mathrm{C}$ & 10 \\
\hline 9 & $\mathrm{CH}_{3} \mathrm{CN}$ & $\left.\left[\mathrm{Rh}\left(\mathrm{Cp}^{*}\right)\left(\mathrm{CH}_{3} \mathrm{CN}\right)_{3}\right)\right]\left[\mathrm{SbF}_{6}\right]_{2}$ & Ad-1-COOH & $110^{\circ} \mathrm{C}$ & NR \\
\hline 10 & $\begin{array}{c}1,4- \\
\text { dioxane }\end{array}$ & $\left.\left[\mathrm{Rh}\left(\mathrm{Cp}^{*}\right)\left(\mathrm{CH}_{3} \mathrm{CN}\right)_{3}\right)\right]\left[\mathrm{SbF}_{6}\right]_{2}$ & Ad-1-COOH & $110^{\circ} \mathrm{C}$ & trace \\
\hline 11 & DME & $\left.\left[\mathrm{Rh}\left(\mathrm{Cp}^{*}\right)\left(\mathrm{CH}_{3} \mathrm{CN}\right)_{3}\right)\right]\left[\mathrm{SbF}_{6}\right]_{2}$ & $\mathrm{Ad}-1-\mathrm{COOH}$ & $110^{\circ} \mathrm{C}$ & trace \\
\hline 12 & $\mathrm{MeOH}$ & $\left.\left[\mathrm{Rh}\left(\mathrm{Cp}^{*}\right)\left(\mathrm{CH}_{3} \mathrm{CN}\right)_{3}\right)\right]\left[\mathrm{SbF}_{6}\right]_{2}$ & Ad-1-COOH & $110^{\circ} \mathrm{C}$ & 30 \\
\hline 13 & TFE & $\left.\left[\mathrm{Rh}\left(\mathrm{Cp}^{*}\right)\left(\mathrm{CH}_{3} \mathrm{CN}\right)_{3}\right)\right]\left[\mathrm{SbF}_{6}\right]_{2}$ & Ad-1-COOH & $110^{\circ} \mathrm{C}$ & 45 \\
\hline 14 & DCE & $\left.\left[\mathrm{Rh}\left(\mathrm{Cp}^{*}\right)\left(\mathrm{CH}_{3} \mathrm{CN}\right)_{3}\right)\right]\left[\mathrm{SbF}_{6}\right]_{2}$ & $\mathrm{Ad}-1-\mathrm{COOH}$ & $110^{\circ} \mathrm{C}$ & trace \\
\hline 15 & $\begin{array}{c}\text { 1,2- } \\
\text { dichloro } \\
\text { benzene }\end{array}$ & $\left.\left[\mathrm{Rh}\left(\mathrm{Cp}^{*}\right)\left(\mathrm{CH}_{3} \mathrm{CN}\right)_{3}\right)\right]\left[\mathrm{SbF}_{6}\right]_{2}$ & $\mathrm{Ad}-1-\mathrm{COOH}$ & $110^{\circ} \mathrm{C}$ & 50 \\
\hline 16 & THF & $\left.\left[\mathrm{Rh}\left(\mathrm{Cp}^{*}\right)\left(\mathrm{CH}_{3} \mathrm{CN}\right)_{3}\right)\right]\left[\mathrm{SbF}_{6}\right]_{2}$ & Ad-1-COOH & r.t & 20 \\
\hline 17 & THF & $\left.\left[\mathrm{Rh}\left(\mathrm{Cp}^{*}\right)\left(\mathrm{CH}_{3} \mathrm{CN}\right)_{3}\right)\right]\left[\mathrm{SbF}_{6}\right]_{2}$ & $\mathrm{Ad}-1-\mathrm{COOH}$ & $60{ }^{\circ} \mathrm{C}$ & 32 \\
\hline 18 & THF & $\left.\left[\mathrm{Rh}\left(\mathrm{Cp}^{*}\right)\left(\mathrm{CH}_{3} \mathrm{CN}\right)_{3}\right)\right]\left[\mathrm{SbF}_{6}\right]_{2}$ & Ad-1-COOH & $80{ }^{\circ} \mathrm{C}$ & 47 \\
\hline 19 & THF & $\left.\left[\mathrm{Rh}\left(\mathrm{Cp}^{*}\right)\left(\mathrm{CH}_{3} \mathrm{CN}\right)_{3}\right)\right]\left[\mathrm{SbF}_{6}\right]_{2}$ & $\mathrm{Ad}-1-\mathrm{COOH}$ & $100{ }^{\circ} \mathrm{C}$ & 68 \\
\hline 20 & THF & $\left.\left[\mathrm{Rh}\left(\mathrm{Cp}^{*}\right)\left(\mathrm{CH}_{3} \mathrm{CN}\right)_{3}\right)\right]\left[\mathrm{SbF}_{6}\right]_{2}$ & Ad-1-COOH & $120{ }^{\circ} \mathrm{C}$ & 71 \\
\hline 21 & THF & $\left.\left[\mathrm{Rh}\left(\mathrm{Cp}^{*}\right)\left(\mathrm{CH}_{3} \mathrm{CN}\right)_{3}\right)\right]\left[\mathrm{SbF}_{6}\right]_{2}$ & Ad-1-COOH & $130{ }^{\circ} \mathrm{C}$ & 70 \\
\hline
\end{tabular}




\section{Crystallographic Data of Compound 6al}

Suitable single crystals for X-ray diffraction studies were obtained from the compound synthesized in this study. Single crystals were grown in an NMR tube at room temperature using $\mathrm{CDCl}_{3}$ over a period of 3-4 weeks by slow evaporation of solvent.

X-ray data was collected with a Bruker AXS (Kappa Apex 2) CCD diffractometer equipped with graphite monochromatic $\mathrm{Mo}(\mathrm{K} \alpha)(\lambda=0.7107 \mathrm{~A})$ radiation source. The data were collected with $100 \%$ completeness for $\Theta$ up to $25^{\circ}$. $\omega$ and $\phi$ scans were employed to collect the data. The frame width for $\omega$ for was fixed to $0.5^{\circ}$ for data collection. The crystal was solved by direct methods using Bruker SHELXS (Sheldrick, 1997). The Structure was refined using the Bruker SHELXTL (Version 6.12) software package. These data were deposited with Cambridge Crystallographic Data Centre with the following numbers: CCDC 2061543.

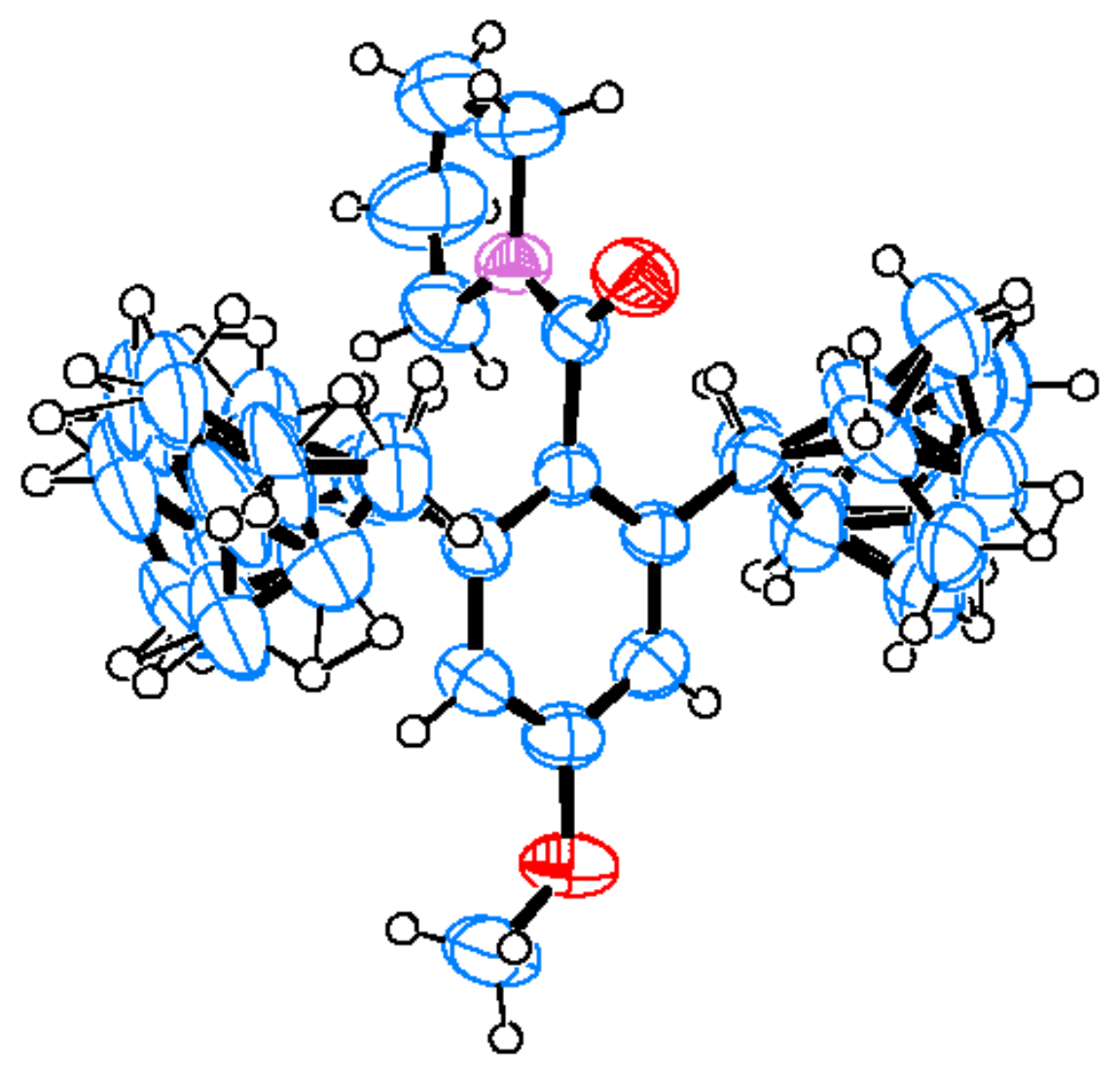

Figure S1: ORTEP representation of compound 6al displaying thermal elliposoid at $50 \%$ probability. 
X-Ray Analysis of Compounds 6al.

\begin{tabular}{|c|c|}
\hline & 6al \\
\hline empirical formula & $\mathrm{C}_{26} \mathrm{H}_{35} \mathrm{NO}_{2}$ \\
\hline formula wt & 393.03 \\
\hline temp $(\mathrm{K})$ & $296(2)$ \\
\hline Cryst. syst. & triclinic \\
\hline space group & P-1 \\
\hline$a(\AA)$ & $8.3825(7)$ \\
\hline$b(\AA)$ & $9.8056(7)$ \\
\hline$c(\AA)$ & $13.9986(10)$ \\
\hline$\alpha(\operatorname{deg})$ & $90.615(4)$ \\
\hline$\beta(\operatorname{deg})$ & $99.217(4)$ \\
\hline$\gamma(\operatorname{deg})$ & $98.609(4)$ \\
\hline$V\left(\AA^{3}\right)$ & $1122.26(15)$ \\
\hline$Z$ & 2 \\
\hline$\rho_{\text {calcd }}\left(\mathrm{Mg} \mathrm{m}^{-3}\right)$ & 1.163 \\
\hline$\mu\left(\mathrm{mm}^{-1}\right)$ & 0.072 \\
\hline$F(000)$ & 427.0 \\
\hline Cryst size (mm) & $0.200 \times 0.180 \times 0.150$ \\
\hline$\Theta$ range (deg) & 2.95 to 49.996 \\
\hline no. of collected/unique & 29204/ 29204 \\
\hline rflns & {$[$ Rsigma $=0.1206]$} \\
\hline $\begin{array}{l}\text { no.of. data /restraints/ } \\
\text { params }\end{array}$ & $29204 / 1130 / 390$ \\
\hline$R 1, w R 2(I>2 \sigma(I))$ & $0.0803,0.2089$ \\
\hline$R 1, w R 2$ (all data) & $0.1863,0.2793$ \\
\hline GOF & 0.956 \\
\hline$\Delta \rho_{\max } / \Delta \rho_{\min }\left(\mathrm{e} \AA^{-3}\right)$ & $0.19 /-0.28$ \\
\hline
\end{tabular}




\section{Reference:}

1) Banister, S.D.; Rendina, L.M.; Kassiou. M. Bioorg Med. Chem. Lett. 2012, 22, 4059.

2) Huestis, M. P. J. Org. Chem. 2016, 81, 12545.

3) Lu, M-Z.; Chen, X-R.; Xu, H.; Dai H-X.; Yu, J-Q. Chem. Sci., 2018, 9, 1311. 


\section{Spectral Data of alkenylation Compounds}

(E)-(2-(Hex-1-en-1-yl)-4-methoxyphenyl)(pyrrolidin-1-yl)methanone/(E)-(2-(Hex-2-en-2yl)-4-methoxyphenyl)(pyrrolidin-1-yl)methanone (3aa/3aa').
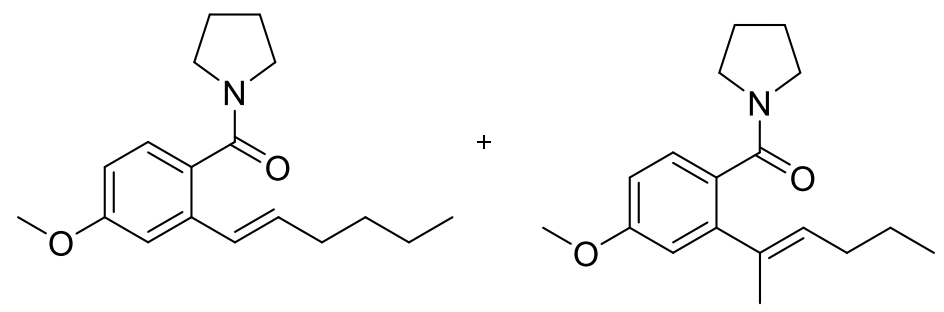

Pale yellow liquid; eluent (hexane, $15 \%$ ethyl acetate). The reaction scale is $50 \mathrm{mg}, 50 \mathrm{mg}$ of product was isolated and yield is $72 \%$.

${ }^{1} \mathrm{H}$ NMR $\left(500 \mathrm{MHz}, \mathrm{CDCl}_{3}\right) \delta 7.18(\mathrm{~d}, \mathrm{~J}=10 \mathrm{~Hz}, 0.5 \mathrm{H}), 7.14(\mathrm{~d}, \mathrm{~J}=8.4 \mathrm{~Hz}, 1 \mathrm{H}), 6.97(\mathrm{~d}, \mathrm{~J}=$ $2.5 \mathrm{~Hz}, 1 \mathrm{H}), 6.77-6.73(\mathrm{~m}, 2 \mathrm{H}), 6.37(\mathrm{dt}, \mathrm{J}=15.7,1.6 \mathrm{~Hz}, 1 \mathrm{H}), 6.19(\mathrm{dt}, \mathrm{J}=15.7,6.9 \mathrm{~Hz}$, $1 \mathrm{H}), 5.53(\mathrm{t}, \mathrm{J}=10 \mathrm{~Hz}, 0.4 \mathrm{H}), 3.80(\mathrm{~s}, 3 \mathrm{H}), 3.79(\mathrm{~s}, 1 \mathrm{H}), 3.62(\mathrm{t}, \mathrm{J}=7.0 \mathrm{~Hz}, 2.7 \mathrm{H}), 3.53(\mathrm{t}, \mathrm{J}=$ $6.9 \mathrm{~Hz}, 1 \mathrm{H}), 3.09(\mathrm{td}, \mathrm{J}=6.7,2.5 \mathrm{~Hz}, 3 \mathrm{H}), 2.17(\mathrm{qd}, \mathrm{J}=7.1,1.5 \mathrm{~Hz}, 2 \mathrm{H}), 2.08(\mathrm{q}, \mathrm{J}=7.3 \mathrm{~Hz}$, $1 \mathrm{H}), 1.96(\mathrm{~d}, \mathrm{~J}=1.3 \mathrm{~Hz}, 1 \mathrm{H}), 1.94-1.86(\mathrm{~m}, 3.7 \mathrm{H}), 1.83-1.75(\mathrm{~m}, 3.9 \mathrm{H}), 1.44-1.37(\mathrm{~m}$, $3.2 \mathrm{H}), 1.36-1.29(\mathrm{~m}, 3 \mathrm{H}), 0.90(\mathrm{dt}, \mathrm{J}=14.4,7.3 \mathrm{~Hz}, 4.9 \mathrm{H})$.

${ }^{13} \mathrm{C}$ NMR $\left(126 \mathrm{MHz}, \mathrm{CDCl}_{3}\right) \delta 170.3,169.8,159.8,159.6,144.0,135.8,134.2,134.0,134.0$, 133.9, 132.2, 131.1, 129.1, 128.9, 128.1, 128.1, 127.9, 127.6, 127.6, 127.6, 127.7, 126.5, 55.2, $48.2,48.1,45.5,32.7,31.3,30.7,29.6,25.9,24.6,24.5,22.5,22.1,17.0,13.9,13.8$.

HRMS (ESI-TOF) m/z: [M + Na $]^{+}$Calcd for $\mathrm{C}_{18} \mathrm{H}_{25} \mathrm{NO}_{2} \mathrm{Na} 310.1783$; Found 310.1795.

(E)-(2-(Hex-1-en-1-yl)-4-methylphenyl)(pyrrolidin-1-yl)methanone/(E)-(2-(Hex-2-en-2yl)-4-methylphenyl)(pyrrolidin-1-yl)methanone (3ba/3ba').
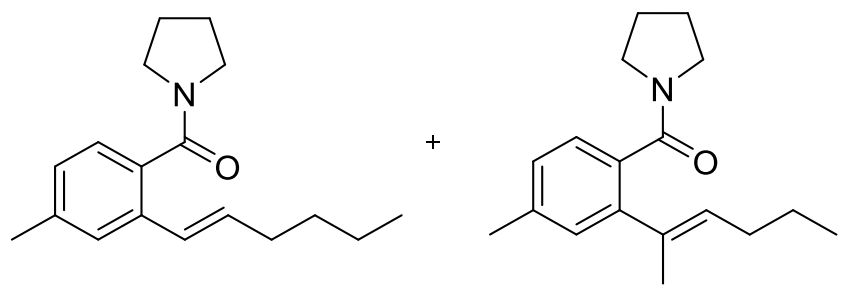

Pale yellow liquid; eluent (hexane, 12\% ethyl acetate). The reaction scale is $50 \mathrm{mg}, 61 \mathrm{mg}$ of product was isolated and yield is $85 \%$.

${ }^{1} \mathrm{H}$ NMR (500 MHz, $\left.\mathrm{CDCl}_{3}\right) \delta 7.29(\mathrm{~d}, \mathrm{~J}=1.7 \mathrm{~Hz}, 1 \mathrm{H}), 7.12(\mathrm{~d}, \mathrm{~J}=7.7 \mathrm{~Hz}, 1 \mathrm{H}), 7.04(\mathrm{td}, \mathrm{J}=$ 7.5, 2.1 Hz, 2H), $6.38(\mathrm{dt}, \mathrm{J}=15.7,1.6 \mathrm{~Hz}, 1 \mathrm{H}), 6.21(\mathrm{dt}, \mathrm{J}=15.7,6.9 \mathrm{~Hz}, 1 \mathrm{H}), 3.65(\mathrm{t}, \mathrm{J}=7.0$ $\mathrm{Hz}, 3 \mathrm{H}), 3.59-3.54(\mathrm{~m}, 1 \mathrm{H}), 3.10(\mathrm{t}, \mathrm{J}=6.8 \mathrm{~Hz}, 3 \mathrm{H}), 2.34(\mathrm{~s}, 4 \mathrm{H}), 2.32(\mathrm{~s}, 1 \mathrm{H}), 2.18$ (qd, J = 7.1, $1.5 \mathrm{~Hz}, 2 \mathrm{H}), 1.98(\mathrm{~d}, \mathrm{~J}=1.3 \mathrm{~Hz}, 1 \mathrm{H}), 1.95-1.88(\mathrm{~m}, 4 \mathrm{H}), 1.85-1.78(\mathrm{~m}, 4 \mathrm{H}), 1.46-$ $1.38(\mathrm{~m}, 3 \mathrm{H}), 1.39-1.31(\mathrm{~m}, 3 \mathrm{H}), 0.92(\mathrm{dt}, \mathrm{J}=14.6,7.3 \mathrm{~Hz}, 5 \mathrm{H})$.

${ }^{13} \mathrm{C}$ NMR $\left(126 \mathrm{MHz}, \mathrm{CDCl}_{3}\right) \delta 170.5,169.9,138.5,138.4,134.3,134.3,133.9,133.9,133.9$, 133.7, 133.6, 133.6, 133.6, 133.5, 133.5, 133.5, 133.5, 133.4, 133.4, 133.4, 133.3, 133.2 131.9, $130.8,129.9,129.3,128.8,128.6,127.7,127.6,127.2,126.6,126.5,126.5,126.3,126.1,126.0$, 
$125.99,48.1,48.1,45.4,34.6,32.8,31.4,31.4,30.7,30.3,29.6,25.8,25.8,24.5,24.5,22.6$, $22.1,21.3,21.2,17.1,13.9,13.8$.

HRMS (ESI-TOF) m/z: [M + Na $]^{+}$Calcd for $\mathrm{C}_{18} \mathrm{H}_{25} \mathrm{NONa} 294.1834$; Found 294.1841.

(E)-(4-Fluoro-2-(hex-1-en-1-yl)phenyl)(pyrrolidin-1-yl)methanone/(4-Fluoro-2-(hex-1en-2-yl)phenyl)(pyrrolidin-1-yl)methanone (3ca/3ca").<smiles>CCCC/C=C/c1cc(F)ccc1C(=O)N1CCCC1</smiles><smiles>C=C(CCCC)c1cc(F)ccc1C(=O)N1CCCC1</smiles>

Pale yellow liquid; eluent (hexane, $12 \%$ ethyl acetate). The reaction scale is $50 \mathrm{mg}, 46 \mathrm{mg}$ of product was isolated and yield is $64 \%$.

${ }^{1} \mathrm{H}$ NMR $\left(400 \mathrm{MHz}, \mathrm{CDCl}_{3}\right) \delta 7.27-7.14(\mathrm{~m}, 2.7 \mathrm{H}), 7.03-6.87(\mathrm{~m}, 2.5 \mathrm{H}), 6.38(\mathrm{~d}, \mathrm{~J}=15.7$ $\mathrm{Hz}, 1 \mathrm{H}), 6.30-6.17(\mathrm{~m}, 1 \mathrm{H}), 5.14(\mathrm{~s}, 0.3 \mathrm{H}), 5.09(\mathrm{~s}, 0.3 \mathrm{H}), 3.65(\mathrm{t}, \mathrm{J}=7.1 \mathrm{~Hz}, 2.5 \mathrm{H}), 3.57$ (t, $\mathrm{J}=6.7 \mathrm{~Hz}, 1 \mathrm{H}), 3.11(\mathrm{dt}, \mathrm{J}=16.7,6.7 \mathrm{~Hz}, 3.5 \mathrm{H}), 2.37(\mathrm{t}, \mathrm{J}=7.5 \mathrm{~Hz}, 0.7 \mathrm{H}), 2.20(\mathrm{q}, \mathrm{J}=7.1$ $\mathrm{Hz}, 2 \mathrm{H}), 1.94(\mathrm{td}, \mathrm{J}=12.1,10.0,7.1 \mathrm{~Hz}, 4.5 \mathrm{H}), 1.84$ (p, J = 7.6, $7.0 \mathrm{~Hz}, 4 \mathrm{H}), 1.39$ (dq, J = 22.3, $7.6 \mathrm{~Hz}, 6 \mathrm{H}), 0.97-0.85(\mathrm{~m}, 5.5 \mathrm{H})$.

${ }^{13} \mathrm{C}$ NMR $\left(101 \mathrm{MHz}, \mathrm{CDCl}_{3}\right) \delta 168.9,164.1,161.6,147.8,136.8,136.3,135.2,132.9,132.3$, 132.3, 132.1, 128.5, 128.4, 128.3, 128.1, 127.9, 127.1, 125.6, 116.3, 116.2, 115.7, 115.5, 114.9, $114.1,114.0,113.9,113.7,112.1,111.8,48.6,48.3,48.1,48.0,45.5,45.4,36.0,32.7,31.2$, $30.7,30.2,29.6,25.9,25.8,24.5,24.4,22.4,22.2,22.1,13.8,13.8,13.6$.

HRMS (ESI-TOF) m/z: [M + Na $]^{+}$Calcd for $\mathrm{C}_{17} \mathrm{H}_{22} \mathrm{FNONa} 298.1583$; Found 298.1592.

(E)-(4-Chloro-2-(hex-1-en-1-yl)phenyl)(pyrrolidin-1-yl)methanone/(E)-(4-Chloro-2(hex-2-en-2-yl)phenyl)(pyrrolidin-1-yl)methanone (3da/3da').<smiles>CCCC/C=C/c1cc(Cl)ccc1C(=O)N1CCCC1</smiles>

Pale yellow liquid; eluent (hexane, $10 \%$ ethyl acetate). The reaction scale is $50 \mathrm{mg}, 55 \mathrm{mg}$ of product was isolated and yield is $79 \%$.

${ }^{1} \mathrm{H}$ NMR (400 MHz, $\left.\mathrm{CDCl}_{3}\right) \delta 7.47(\mathrm{~d}, \mathrm{~J}=1.9 \mathrm{~Hz}, 1 \mathrm{H}), 7.30-7.10(\mathrm{~m}, 4.75 \mathrm{H}), 6.35(\mathrm{~d}, \mathrm{~J}=$ $15.9 \mathrm{~Hz}, 1 \mathrm{H}), 6.25(\mathrm{dt}, \mathrm{J}=15.6,6.7 \mathrm{~Hz}, 1 \mathrm{H}), 5.57(\mathrm{t}, \mathrm{J}=10 \mathrm{~Hz}, 0.3 \mathrm{H}), 3.64(\mathrm{t}, \mathrm{J}=7.0 \mathrm{~Hz}$, 2.7H), $3.56(\mathrm{~d}, \mathrm{~J}=6.3 \mathrm{~Hz}, 1 \mathrm{H}), 3.11(\mathrm{dt}, \mathrm{J}=16.3,6.7 \mathrm{~Hz}, 3.7 \mathrm{H}), 2.20(\mathrm{q}, \mathrm{J}=7.0 \mathrm{~Hz}, 2 \mathrm{H}), 2.14$ $-2.06(\mathrm{~m}, 1 \mathrm{H}), 1.95(\mathrm{dd}, \mathrm{J}=13.4,6.5 \mathrm{~Hz}, 5 \mathrm{H}), 1.84(\mathrm{p}, \mathrm{J}=6.9 \mathrm{~Hz}, 4 \mathrm{H}), 1.38(\mathrm{dp}, \mathrm{J}=22.0,7.4$ $\mathrm{Hz}, 6 \mathrm{H}), 0.92(\mathrm{dt}, \mathrm{J}=11.2,7.3 \mathrm{~Hz}, 4.7 \mathrm{H})$. 
${ }^{13} \mathrm{C} \mathrm{NMR}\left(101 \mathrm{MHz}, \mathrm{CDCl}_{3}\right) \delta 169.1,168.7,143.8,136.0,135.4,134.7,134.5,133.2,133.0$, 132.2, 128.7, 128.1, 128.0, 127.8, 127.5, 127.2, 126.8, 126.6, 126.2, 125.9, 125.5, 125.3, 119.9, $115.5,48.6,48.1,48.0,45.5,45.4,36.0,35.9,32.7,31.2,30.7,29.6,25.9,25.9,24.5,24.4$, $22.6,22.5,22.3,22.1,16.9,13.8,13.8,13.6$

HRMS (ESI-TOF) m/z: [M + Na $]^{+}$Calcd for $\mathrm{C}_{17} \mathrm{H}_{22} \mathrm{ClNONa} 314.1288$; Found 314.1296.

(E)-(4-Bromo-2-(hex-1-en-1-yl)phenyl)(pyrrolidin-1-yl)methanone/(E)-(4-Bromo-2-(hex2-en-2-yl)phenyl)(pyrrolidin-1-yl)methanone (3ea/3ea').<smiles>CCCC/C=C/c1cc(Br)ccc1C(=O)N1CCCC1</smiles><smiles>CCC/C=C(\C)c1cc(Br)ccc1C(=O)N1CCCC1</smiles>

Pale yellow liquid; eluent (hexane, $10 \%$ ethyl acetate). The reaction scale is $50 \mathrm{mg}, 58 \mathrm{mg}$ of product was isolated and yield is $87 \%$.

${ }^{1} \mathrm{H}$ NMR $\left(400 \mathrm{MHz}, \mathrm{CDCl}_{3}\right) \delta 7.63(\mathrm{~s}, 1 \mathrm{H}), 7.45-7.31(\mathrm{~m}, 2.7 \mathrm{H}), 7.10(\mathrm{td}, \mathrm{J}=13.5,11.9,7.9$ $\mathrm{Hz}, 1.8 \mathrm{H}), 6.33(\mathrm{~d}, \mathrm{~J}=15.9 \mathrm{~Hz}, 1 \mathrm{H}), 6.29-6.20(\mathrm{~m}, 1 \mathrm{H}), 5.56(\mathrm{t}, 10 \mathrm{~Hz}, 0.4 \mathrm{H}), 3.64(\mathrm{t}, \mathrm{J}=7.1$ $\mathrm{Hz}, 2.8 \mathrm{H}), 3.56(\mathrm{~d}, \mathrm{~J}=6.7 \mathrm{~Hz}, 1 \mathrm{H}), 3.11(\mathrm{dt}, \mathrm{J}=17.1,6.7 \mathrm{~Hz}, 3.7 \mathrm{H}), 2.35(\mathrm{t}, \mathrm{J}=7.4 \mathrm{~Hz}, 0.8 \mathrm{H})$, $2.20(\mathrm{q}, \mathrm{J}=7.0 \mathrm{~Hz}, 2.2 \mathrm{H}), 2.03-1.90(\mathrm{~m}, 5.5 \mathrm{H}), 1.88-1.77(\mathrm{~m}, 4.7 \mathrm{H}), 1.38(\mathrm{dp}, \mathrm{J}=22.3,7.5$ $\mathrm{Hz}, 7 \mathrm{H}), 0.91(\mathrm{t}, \mathrm{J}=7.2 \mathrm{~Hz}, 5.2 \mathrm{H})$.

${ }^{13} \mathrm{C}$ NMR $\left(101 \mathrm{MHz}, \mathrm{CDCl}_{3}\right) \delta 168.8,168.7,148.0,142.1,136.3,135.5,135.0,134.9,132.9$, 132.3, 131.6, 131.1, 130.2, 129.7, 129.6, 129.2, 128.5, 128.2, 128.0, 127.7, 127.4, 125.2, 123.0, $115.92,107.1,67.5,48.6,48.3,48.1,45.5,45.4,36.4,36.0,32.7,31.2,30.2$, 29.6, 29.1, 27.8 , $25.8,24.5,24.4,23.8,22.5,22.3,22.1,13.9,13.8$.

HRMS (ESI-TOF) m/z: [M + Na $]^{+}$Calcd for $\mathrm{C}_{17} \mathrm{H}_{22} \mathrm{BrNONa} 358.0782$; Found 358.0789.

(E)-(2-(Hex-1-en-1-yl)-4-nitrophenyl)(pyrrolidin-1-yl)methanone/(E)-(2-(Hex-2-en-2-yl)4-nitrophenyl)(pyrrolidin-1-yl)methanone (3fa/3fa").<smiles>CCCC/C=C/c1cc([N+](=O)[O-])ccc1C(=O)N1CCCC1</smiles><smiles>C=C(CCCC)c1cc([N+](=O)[O-])ccc1C(=O)N1CCCC1</smiles>

Pale yellow liquid; eluent (hexane, 12\% ethyl acetate). The reaction scale is $50 \mathrm{mg}, 40 \mathrm{mg}$ of product was isolated and yield is $58 \%$.

${ }^{1} \mathrm{H}$ NMR (400 MHz, $\left.\mathrm{CDCl}_{3}\right) \delta 8.29(\mathrm{~d}, \mathrm{~J}=2.2 \mathrm{~Hz}, 1 \mathrm{H}), 8.07(\mathrm{dd}, \mathrm{J}=7.5,5.3 \mathrm{~Hz}, 1.8 \mathrm{H}), 8.04$ $-7.95(\mathrm{~m}, 1.5 \mathrm{H}), 7.34(\mathrm{dt}, \mathrm{J}=17.5,8.6 \mathrm{~Hz}, 2 \mathrm{H}), 6.35(\mathrm{~d}, \mathrm{~J}=2.5 \mathrm{~Hz}, 2 \mathrm{H}), 5.18(\mathrm{~s}, 0.6 \mathrm{H}), 5.09$ $(\mathrm{s}, 0.6 \mathrm{H}), 3.60(\mathrm{q}, \mathrm{J}=7.1 \mathrm{~Hz}, 3 \mathrm{H}), 3.53(\mathrm{t}, \mathrm{J}=7.0 \mathrm{~Hz}, 1.3 \mathrm{H}), 3.39(\mathrm{dd}, \mathrm{J}=13.6,6.7 \mathrm{~Hz}, 1.3 \mathrm{H})$, $3.03(\mathrm{dt}, \mathrm{J}=14.2,6.7 \mathrm{~Hz}, 4.5 \mathrm{H}), 2.35(\mathrm{~d}, \mathrm{~J}=7.3 \mathrm{~Hz}, 1.2 \mathrm{H}), 2.18(\mathrm{q}, \mathrm{J}=6.6 \mathrm{~Hz}, 2.3 \mathrm{H}), 1.96-$ 
$1.86(\mathrm{~m}, 5 \mathrm{H}), 1.85-1.76(\mathrm{~m}, 5 \mathrm{H}), 1.43-1.26(\mathrm{~m}, 8.6 \mathrm{H}), 1.13(\mathrm{~d}, \mathrm{~J}=7.0 \mathrm{~Hz}, 1.3 \mathrm{H}), 0.84(\mathrm{dt}$, $\mathrm{J}=12.2,7.0 \mathrm{~Hz}, 7 \mathrm{H})$.

${ }^{13} \mathrm{C}$ NMR $\left(101 \mathrm{MHz}, \mathrm{CDCl}_{3}\right) \delta 167.4,148.2,147.3,137.4,136.1,134.0,127.7,127.4,126.9$, 126.1, 124.5, 123.8, 122.2, 121.6, 121.5, 120.7, 116.3, 48.5, 48.2, 48.0, 45.7, 45.6, 36.0, 32.8, $31.1,30.2,29.6,25.9,24.5,24.3,22.3,22.2,13.8$.

HRMS (ESI-TOF) m/z: $[\mathrm{M}+\mathrm{Na}]^{+}$Calcd for $\mathrm{C}_{17} \mathrm{H}_{22} \mathrm{ClN}_{2} \mathrm{O}_{3} \mathrm{Na}$ 325.1528; Found 325.1536.

$(E)-(2-(H e x-1-e n-1-y l) p h e n y l)(p y r r o l i d i n-1-y l) m e t h a n o n e /(E)-(2-(H e x-2-e n-2-$ yl)phenyl)(pyrrolidin-1-yl)methanone (3ga/3ga').<smiles>CCCC/C=C/c1ccccc1C(=O)N1CCCC1</smiles><smiles>CCC/C=C(\C)c1ccccc1C(=O)N1CCCC1</smiles>

Pale yellow liquid; eluent (hexane, $10 \%$ ethyl acetate). The reaction scale is $50 \mathrm{mg}, 44 \mathrm{mg}$ of product was isolated and yield is $60 \%$.

${ }^{1} \mathrm{H}$ NMR $\left(400 \mathrm{MHz}, \mathrm{CDCl}_{3}\right) \delta 7.49(\mathrm{~d}, \mathrm{~J}=7.9 \mathrm{~Hz}, 1 \mathrm{H}), 7.37-7.24(\mathrm{~m}, 5.3 \mathrm{H}), 6.40(\mathrm{~d}, \mathrm{~J}=15.8$ $\mathrm{Hz}, 1 \mathrm{H}), 6.22(\mathrm{dt}, \mathrm{J}=15.0,6.8 \mathrm{~Hz}, 1 \mathrm{H}), 5.55(\mathrm{t}, \mathrm{J}=8 \mathrm{~Hz}, 0.4 \mathrm{H}), 3.66(\mathrm{t}, \mathrm{J}=7.1 \mathrm{~Hz}, 3 \mathrm{H}), 3.61$ $-3.55(\mathrm{~m}, 1.6 \mathrm{H}), 3.18-3.06(\mathrm{~m}, 4.6 \mathrm{H}), 2.19(\mathrm{q}, \mathrm{J}=7.2 \mathrm{~Hz}, 2 \mathrm{H}), 2.11(\mathrm{q}, \mathrm{J}=7.5 \mathrm{~Hz}, 1.3 \mathrm{H})$, $2.00(\mathrm{~s}, 2 \mathrm{H}), 1.97-1.90(\mathrm{~m}, 4.8 \mathrm{H}), 1.88-1.79(\mathrm{~m}, 6 \mathrm{H}), 1.42(\mathrm{q}, \mathrm{J}=7.2 \mathrm{~Hz}, 3 \mathrm{H}), 1.35$ (q, J = 7.7, $7.3 \mathrm{~Hz}, 3 \mathrm{H}), 0.93(\mathrm{dt}, \mathrm{J}=14.6,7.4 \mathrm{~Hz}, 5.3 \mathrm{H})$.

${ }^{13} \mathrm{C} \mathrm{NMR}\left(101 \mathrm{MHz}, \mathrm{CDCl}_{3}\right) \delta 170.3,169.7,142.2,136.2,136.1,134.2,134.1,133.9,132.1$, 131.2, 129.7, 128.8, 128.7, 128.7, 128.6, 128.1, 128.1, 126.9, 126.6, 126.6, 126.5, 126.5, 126.4, $126.1,125.8,125.8,125.6,48.6,48.5,48.3,48.1,48.1,45.4,45.3,32.8,31.4,30.7,26.0,25.9$, $24.6,24.5,22.6,22.1,17.2,13.9,13.8$.

HRMS (ESI-TOF) m/z: [M + Na $]^{+}$Calcd for $\mathrm{C}_{18} \mathrm{H}_{25} \mathrm{NONa} 280.1677$; Found 280.1685.

(E)-(2-(Hex-1-en-1-yl)-6-methoxyphenyl)(pyrrolidin-1-yl)methanone/(2-(Hex-1-en-2-yl)6-methoxyphenyl)(pyrrolidin-1-yl)methanone (3ha/3ha').
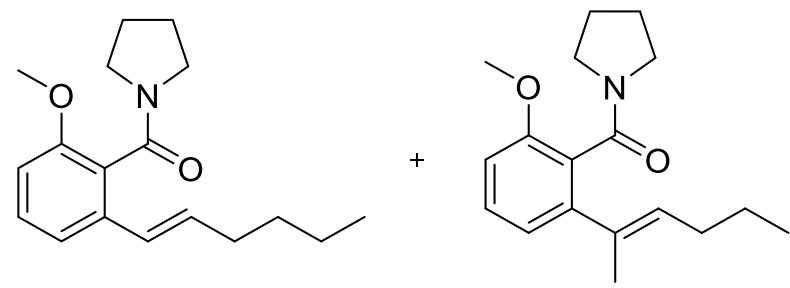

Pale yellow liquid; eluent (hexane, $15 \%$ ethyl acetate). The reaction scale is $50 \mathrm{mg}, 30 \mathrm{mg}$ of product was isolated and yield is $43 \%$.

${ }^{1} \mathrm{H}$ NMR $\left(400 \mathrm{MHz}, \mathrm{CDCl}_{3}\right) \delta 7.24(\mathrm{dd}, \mathrm{J}=16.1,8.0 \mathrm{~Hz}, 2.6 \mathrm{H}), 7.11(\mathrm{~d}, \mathrm{~J}=7.9 \mathrm{~Hz}, 1 \mathrm{H}), 6.86$ $-6.79(\mathrm{~m}, 1.6 \mathrm{H}), 6.76(\mathrm{~d}, \mathrm{~J}=8.4 \mathrm{~Hz}, 1.5 \mathrm{H}), 6.32(\mathrm{~d}, \mathrm{~J}=15.8 \mathrm{~Hz}, 1 \mathrm{H}), 6.22(\mathrm{dt}, \mathrm{J}=15.4,6.6$ $\mathrm{Hz}, 1 \mathrm{H}), 5.47(\mathrm{~d}, \mathrm{~J}=4.7 \mathrm{~Hz}, 0.4 \mathrm{H}), 3.81(\mathrm{~s}, 5.8 \mathrm{H}), 3.73(\mathrm{dt}, \mathrm{J}=13.1,6.8 \mathrm{~Hz}, 2 \mathrm{H}), 3.67-3.59$ 
(m, 1.6H), $3.52-3.45(\mathrm{~m}, 0.8 \mathrm{H}), 3.15-3.02(\mathrm{~m}, 3.7 \mathrm{H}), 2.17(\mathrm{q}, \mathrm{J}=7.0 \mathrm{~Hz}, 2.5 \mathrm{H}), 1.84$ (ddt, $\mathrm{J}=21.5,13.7,6.4 \mathrm{~Hz}, 7 \mathrm{H}), 1.38(\mathrm{ddt}, \mathrm{J}=28.0,14.4,7.4 \mathrm{~Hz}, 7.8 \mathrm{H}), 0.93-0.87$ (m, 5.5H).

${ }^{13} \mathrm{C} \mathrm{NMR}\left(101 \mathrm{MHz}, \mathrm{CDCl}_{3}\right) \delta 167.3,155.3,155.2,144.1,135.6,134.4,134.2,131.99,130.3$, 129.4, 129.3, 129.2, 127.9, 126.1, 125.6, 125.5, 121.7, 121.1, 120.8, 117.7, 113.9, 109.4, 109.0, 108.9, 108.4, 55.7, 55.6, 47.3, 47.2, 45.2, 45.1, 36.7, 36.1 34.6, 32.8, 31.3, 30.5, 29.6, 25.7, 25.7, 24.6, 24.6, 24.6, 22.7, 22.5, 22.3, 22.1, 17.6, 13.9, 13.9, 13.7.

HRMS (ESI-TOF) m/z: [M + Na] ${ }^{+}$Calcd for $\mathrm{C}_{18} \mathrm{H}_{25} \mathrm{NO}_{2} \mathrm{Na} 310.1783$; Found 310.1787.

(E)-2-(Hex-1-en-1-yl)-N,N,4-trimethylbenzamide/2-(Hept-1-en-2-yl)-N,N,4trimethylbenzamide (3ia/3ia")<smiles>CCC/C=C(\C)c1cc(C)ccc1C(=O)N(C)C</smiles>

Pale yellow liquid; eluent (hexane, $10 \%$ ethyl acetate). The reaction scale is $50 \mathrm{mg}, 38 \mathrm{mg}$ of product was isolated and yield is $51 \%$.

${ }^{1} \mathrm{H}$ NMR $\left(400 \mathrm{MHz}, \mathrm{CDCl}_{3}\right) \delta 7.30(\mathrm{~s}, 1 \mathrm{H}), 7.13-7.01(\mathrm{~m}, 4 \mathrm{H}), 6.32(\mathrm{~d}, J=15.8 \mathrm{~Hz}, 1 \mathrm{H})$, $6.20(\mathrm{dt}, J=15.6,6.8 \mathrm{~Hz}, 1 \mathrm{H}), 5.49(\mathrm{t}, \mathrm{J}=4 \mathrm{~Hz}, 0.4 \mathrm{H}), 3.12(\mathrm{~s}, 3.4 \mathrm{H}), 2.77(\mathrm{~s}, 4 \mathrm{H}), 2.34(\mathrm{~s}, 5 \mathrm{H})$, $2.18(\mathrm{q}, J=7.0 \mathrm{~Hz}, 2 \mathrm{H}), 1.47-1.34(\mathrm{~m}, 4.7 \mathrm{H}), 0.91(\mathrm{t}, J=7.2 \mathrm{~Hz}, 4 \mathrm{H})$.

${ }^{13} \mathrm{C}$ NMR $\left(101 \mathrm{MHz}, \mathrm{CDCl}_{3}\right) \delta 172.08,171.61,138.52,138.46,134.35,133.59,132.26$, $130.84,128.55,127.65,127.27,126.84,126.40,126.26,125.96,125.92,77.32,77.00,76.68$, $38.64,38.59,38.44,36.42,34.68,34.60,32.81,31.40,30.66,27.83,22.62,22.15,21.29,21.23$, $17.15,13.86$.

HRMS (ESI-TOF) m/z: $[\mathrm{M}+\mathrm{Na}]^{+}$Calcd for $\mathrm{C}_{16} \mathrm{H}_{23} \mathrm{NONa} 268.1677$; Found 268.1681.

$(E)$-(2-(Hex-1-en-1-yl)-5-methylphenyl)(pyrrolidin-1-yl)methanone/(E)-(2-(Hex-2-en-2yl)-5-methylphenyl)(pyrrolidin-1-yl)methanone (3ja/3ja').<smiles>CCCC/C=C/c1ccc(C)cc1C(=O)N1CCCC1</smiles>

Pale yellow liquid; eluent (hexane, $12 \%$ ethyl acetate). The reaction scale is $50 \mathrm{mg}, 51 \mathrm{mg}$ of product was isolated and yield is $71 \%$.

${ }^{1} \mathrm{H}$ NMR (400 MHz, $\left.\mathrm{CDCl}_{3}\right) \delta 7.35(\mathrm{~d}, \mathrm{~J}=8.0 \mathrm{~Hz}, 1 \mathrm{H}), 7.09(\mathrm{q}, \mathrm{J}=8.8,7.8 \mathrm{~Hz}, 2.8 \mathrm{H}), 7.02(\mathrm{~s}$, $1 \mathrm{H}), 6.33(\mathrm{~d}, \mathrm{~J}=15.8 \mathrm{~Hz}, 1 \mathrm{H}), 6.15(\mathrm{dt}, \mathrm{J}=15.0,6.9 \mathrm{~Hz}, 1 \mathrm{H}), 5.52(\mathrm{t}, \mathrm{J}=8 \mathrm{~Hz}, 0.3 \mathrm{H}), 3.63$ $(\mathrm{t}, \mathrm{J}=7.0 \mathrm{~Hz}, 2.5 \mathrm{H}), 3.09(\mathrm{t}, \mathrm{J}=6.8 \mathrm{~Hz}, 2.7 \mathrm{H}), 2.30(\mathrm{~s}, 4.4 \mathrm{H}), 2.16(\mathrm{q}, \mathrm{J}=7.1 \mathrm{~Hz}, 2 \mathrm{H}), 1.96$ (s, 1H), $1.95-1.88(\mathrm{~m}, 3.5 \mathrm{H}), 1.85-1.77(\mathrm{~m}, 3.6 \mathrm{H}), 1.38(\mathrm{ddt}, \mathrm{J}=19.7,14.5,7.2 \mathrm{~Hz}, 5.6 \mathrm{H})$, $0.89(\mathrm{t}, \mathrm{J}=7.0 \mathrm{~Hz}, 4.5 \mathrm{H})$. 
${ }^{13} \mathrm{C} \mathrm{NMR}\left(101 \mathrm{MHz}, \mathrm{CDCl}_{3}\right) \delta 170.4,169.9,139.3,136.7,136.3,136.1,132.9,131.2,130.7$, 129.6, 129.4, 128.6, 127.9, 127.2, 126.5, 126.3, 125.5, 48.0, 47.9, 45.4, 45.4, 32.8, 31.4, 30.74, $29.6,25.9,24.6,22.6,22.1,20.9,20.8,17.1,13.9$.

HRMS (ESI-TOF) m/z: [M + Na] ${ }^{+}$Calcd for $\mathrm{C}_{18} \mathrm{H}_{25} \mathrm{NONa} 294.1834$; Found 294.1840.

(E)-(5-Bromo-2-(hex-1-en-1-yl)phenyl)(pyrrolidin-1-yl)methanone/(5-Bromo-2-(hex-1en-2-yl)phenyl)(pyrrolidin-1-yl)methanone (3ka/3ka").<smiles>CCCC/C=C/c1ccc(Br)cc1C(=O)N1CCCC1</smiles><smiles>C=C(CCCC)c1ccc(Br)cc1C(=O)N1CCCC1</smiles>

Pale yellow liquid; eluent (hexane, $10 \%$ ethyl acetate). The reaction scale is $50 \mathrm{mg}, 42 \mathrm{mg}$ of product was isolated and yield is $64 \%$.

${ }^{1} \mathrm{H}$ NMR $\left(400 \mathrm{MHz}, \mathrm{CDCl}_{3}\right) \delta 7.45-7.41(\mathrm{~m}, 1 \mathrm{H}), 7.37(\mathrm{~d}, \mathrm{~J}=15.6 \mathrm{~Hz}, 3 \mathrm{H}), 7.32(\mathrm{~d}, \mathrm{~J}=4.3$ $\mathrm{Hz}, 0.6 \mathrm{H}), 7.11(\mathrm{dd}, \mathrm{J}=8.5,2.5 \mathrm{~Hz}, 0.7 \mathrm{H}), 6.31(\mathrm{~d}, \mathrm{~J}=15.8 \mathrm{~Hz}, 1 \mathrm{H}), 6.21(\mathrm{dt}, \mathrm{J}=15.6,6.6$ $\mathrm{Hz}, 1 \mathrm{H}), 5.11(\mathrm{~s}, 0.4 \mathrm{H}), 5.04(\mathrm{~s}, 0.4 \mathrm{H}), 3.62(\mathrm{q}, \mathrm{J}=7.9,7.4 \mathrm{~Hz}, 2.8 \mathrm{H}), 3.55(\mathrm{q}, \mathrm{J}=6.1,5.3$ $\mathrm{Hz}, 1.4 \mathrm{H}), 3.14(\mathrm{t}, \mathrm{J}=6.7 \mathrm{~Hz}, 1.4 \mathrm{H}), 3.10(\mathrm{t}, \mathrm{J}=6.7 \mathrm{~Hz}, 2.2 \mathrm{H}), 2.34(\mathrm{t}, \mathrm{J}=7.4 \mathrm{~Hz}, 0.8 \mathrm{H}), 2.17$ $(\mathrm{q}, \mathrm{J}=7.0 \mathrm{~Hz}, 2 \mathrm{H}), 1.97-1.91(\mathrm{~m}, 3.8 \mathrm{H}), 1.87-1.80(\mathrm{~m}, 4 \mathrm{H}), 1.39(\mathrm{q}, \mathrm{J}=7.4 \mathrm{~Hz}, 3.2 \mathrm{H}), 1.33$ $(\mathrm{td}, \mathrm{J}=9.9,8.5,5.7 \mathrm{~Hz}, 4.5 \mathrm{H}), 0.92-0.81(\mathrm{~m}, 6.7 \mathrm{H})$.

${ }^{13} \mathrm{C} \mathrm{NMR}\left(101 \mathrm{MHz}, \mathrm{CDCl}_{3}\right) \delta 182.9,168.2,168.1,148.2,139.0,137.8,137.7,134.9,133.2$, 132.8, 132.0, 131.8, 131.6, 130.5, 129.4, 129.0, 128.7, 127.4, 127.4, 125.5, 121.0, 120.5, 114.9, 107.9, 67.7, 48.7, 48.4, 48.2, 45.6, 45.5, 40.4, 38.6, 36.4, 36.2, 35.6, 34.6, 32.9, 31.3, 30.8, 30.3, 29.7, 29.1, 27.8, 27.7, 26.0, 25.9, 24.6, 24.5, 23.9, 22.5, 22.3, 22.2, 14.1, 13.9, 13.7.

HRMS (ESI-TOF) m/z: [M + Na $]^{+}$Calcd for $\mathrm{C}_{17} \mathrm{H}_{22} \mathrm{BrNONa}$ 358.0782; Found 358.0790.

(E)-(5-Chloro-2-(hex-1-en-1-yl)phenyl)(pyrrolidin-1-yl)methanone/(5-Chloro-2-(hex-1en-2-yl)phenyl)(pyrrolidin-1-yl)methanone (3la/3la").<smiles>CCCC/C=C/c1ccc(Cl)cc1C(=O)N1CCCC1</smiles><smiles>C=C(CCCC)c1ccc(Cl)cc1C(=O)N1CCCC1</smiles>

Pale yellow liquid; eluent (hexane, $12 \%$ ethyl acetate). The reaction scale is $50 \mathrm{mg}, 35 \mathrm{mg}$ of product was isolated and yield is $50 \%$.

${ }^{1} \mathrm{H}$ NMR $\left(400 \mathrm{MHz}, \mathrm{CDCl}_{3}\right) \delta 7.41(\mathrm{~d}, \mathrm{~J}=8.4 \mathrm{~Hz}, 1 \mathrm{H}), 7.24(\mathrm{~s}, 1.3 \mathrm{H}), 7.21-7.16(\mathrm{~m}, 1.9 \mathrm{H})$, $6.32(\mathrm{~d}, \mathrm{~J}=15.8 \mathrm{~Hz}, 1 \mathrm{H}), 6.20(\mathrm{dt}, \mathrm{J}=15.5,6.8 \mathrm{~Hz}, 1 \mathrm{H}), 5.12(\mathrm{~s}, 0.2 \mathrm{H}), 5.04(\mathrm{~s}, 0.2 \mathrm{H}), 3.64$ $(\mathrm{t}, \mathrm{J}=7.0 \mathrm{~Hz}, 2.8 \mathrm{H}), 3.55(\mathrm{~d}, \mathrm{~J}=6.1 \mathrm{~Hz}, 1.1 \mathrm{H}), 3.14(\mathrm{t}, \mathrm{J}=6.7 \mathrm{~Hz}, 1.2 \mathrm{H}), 3.10(\mathrm{t}, \mathrm{J}=6.7 \mathrm{~Hz}$, 2.8H), 2.17 (q, J = 7.0 Hz, 2.1H), $1.95(\mathrm{~d}, \mathrm{~J}=7.4 \mathrm{~Hz}, 4.2 \mathrm{H}), 1.85$ (d, J = 6.9 Hz, 4.3H), 1.40 $(\mathrm{q}, \mathrm{J}=7.4 \mathrm{~Hz}, 3.2 \mathrm{H}), 1.36-1.29(\mathrm{~m}, 3.8 \mathrm{H}), 0.89$ (q, J = 6.8 Hz, 5.9H). 
${ }^{13} \mathrm{C} \mathrm{NMR}\left(101 \mathrm{MHz}, \mathrm{CDCl}_{3}\right) \delta 168.2,137.4,134.7,132.7,132.5,130.3,129.6,129.1,127.1$, 126.2, 125.4, 114.9, 70.3, 48.4, 48.1, 45.6, 45.5, 40.4, 38.6, 36.4, 36.2, 34.6, 32.9, 31.3, 30.8, $30.3,29.7,27.8,27.8,26.4,26.0,25.9,24.6,24.5,22.6,22.3,22.2,13.9,13.9$.

HRMS (ESI-TOF) m/z: [M + Na $]^{+}$Calcd for $\mathrm{C}_{17} \mathrm{H}_{22} \mathrm{CINONa} 314.1288$; Found 314.1292.

(E)-(5-Fuoro-2-(hex-1-en-1-yl)phenyl)(pyrrolidin-1-yl)methanone/(E)-(5-Fluoro-2-(hex2-en-2-yl)phenyl)(pyrrolidin-1-yl)methanone (3ma/3ma').<smiles>CCCC/C=C/c1ccc(F)cc1C(=O)N1CCCC1</smiles><smiles>CCC/C=C(\C)c1ccc(F)cc1C(=O)N1CCCC1</smiles>

Pale yellow liquid; eluent (hexane, $12 \%$ ethyl acetate). The reaction scale is $50 \mathrm{mg}, 26 \mathrm{mg}$ of product was isolated and yield is $37 \%$.

${ }^{1} \mathrm{H}$ NMR $\left(400 \mathrm{MHz}, \mathrm{CDCl}_{3}\right) \delta 7.19(\mathrm{td}, \mathrm{J}=7.7,4.2 \mathrm{~Hz}, 1.8 \mathrm{H}), 7.02(\mathrm{q}, \mathrm{J}=8.8,8.2 \mathrm{~Hz}, 3.3 \mathrm{H})$, $6.32(\mathrm{~d}, \mathrm{~J}=5.7 \mathrm{~Hz}, 2 \mathrm{H}), 5.47(\mathrm{~d}, \mathrm{~J}=4.7 \mathrm{~Hz}, 0.7 \mathrm{H}), 3.63(\mathrm{q}, \mathrm{J}=7.3 \mathrm{~Hz}, 3.8 \mathrm{H}), 3.40$ (t, J = 6.1 $\mathrm{Hz}, 1.3 \mathrm{H}), 3.13(\mathrm{t}, \mathrm{J}=7.0 \mathrm{~Hz}, 1 \mathrm{H}), 3.08(\mathrm{t}, \mathrm{J}=6.7 \mathrm{~Hz}, 2.1 \mathrm{H}), 2.20(\mathrm{q}, \mathrm{J}=6.2 \mathrm{~Hz}, 2.2 \mathrm{H}), 1.93$ $(\mathrm{p}, \mathrm{J}=7.5 \mathrm{~Hz}, 5.7 \mathrm{H}), 1.85(\mathrm{q}, \mathrm{J}=6.8 \mathrm{~Hz}, 4.4 \mathrm{H}), 1.45-1.32(\mathrm{~m}, 5.7 \mathrm{H}), 0.91(\mathrm{t}, \mathrm{J}=7.1 \mathrm{~Hz}$, $3.6 \mathrm{H}), 0.86(\mathrm{t}, \mathrm{J}=7.3 \mathrm{~Hz}, 1.8 \mathrm{H})$.

${ }^{13} \mathrm{C}$ NMR $\left(101 \mathrm{MHz}, \mathrm{CDCl}_{3}\right) \delta 168.7,168.7,138.7,138.6,138.4,131.9,130.1,130.0,128.1$, 128.0, 127.8, 126.7, 122.8, 122.8, 122.1, 122.1, 121.5, 120.0, 116.2, 115.9, 115.7, 114.3, 49.6, $48.7,47.9,46.3,45.6,45.5,34.6,33.7,31.3,29.7,29.4,26.4,25.9,25.9,24.6,24.6,24.4,22.5$, 22.2 .

HRMS (ESI-TOF) m/z: [M + Na] ${ }^{+}$Calcd for $\mathrm{C}_{17} \mathrm{H}_{22} \mathrm{FNONa} 298.1583$; Found 298.1588.

(E)-(2-(Hex-1-en-1-yl)-4,5-dimethylphenyl)(pyrrolidin-1-yl)methanone/(E)-(2-(Hex-2en-2-yl)-4,5-dimethylphenyl)(pyrrolidin-1-yl)methanone (3na/3na')<smiles>CCCC/C=C/c1cc(C)c(C)cc1C(=O)N1CCCC1</smiles>

Pale yellow liquid; eluent (hexane, $10 \%$ ethyl acetate). The reaction scale is $50 \mathrm{mg}, 46 \mathrm{mg}$ of product was isolated and yield is $65 \%$.

${ }^{1} \mathrm{H}$ NMR $\left(400 \mathrm{MHz}, \mathrm{CDCl}_{3}\right) \delta 7.23(\mathrm{~s}, 1 \mathrm{H}), 6.97(\mathrm{~s}, 1.9 \mathrm{H}), 6.32(\mathrm{~d}, J=15.8 \mathrm{~Hz}, 1 \mathrm{H}), 6.20-$ $6.08(\mathrm{~m}, 1 \mathrm{H}), 5.50(\mathrm{t}, J=7.6 \mathrm{~Hz}, 0.5 \mathrm{H}), 3.63(\mathrm{t}, J=7.0 \mathrm{~Hz}, 3 \mathrm{H}), 3.54(\mathrm{t}, J=6.9 \mathrm{~Hz}, 1 \mathrm{H}), 3.15$ $(\mathrm{d}, J=6.7 \mathrm{~Hz}, 1.3 \mathrm{H}), 3.10(\mathrm{t}, J=6.7 \mathrm{~Hz}, 3 \mathrm{H}), 2.23(\mathrm{~s}, 5.1 \mathrm{H}), 2.21(\mathrm{~s}, 3.4 \mathrm{H}), 2.20(\mathrm{~s}, 1.8 \mathrm{H})$, $1.94-1.89(\mathrm{~m}, 3.8 \mathrm{H}), 1.82(\mathrm{dd}, J=13.7,6.9 \mathrm{~Hz}, 5.8 \mathrm{H}), 1.39(\mathrm{dd}, J=14.7,7.3 \mathrm{~Hz}, 3.8 \mathrm{H}), 1.33$ $(\mathrm{dd}, J=14.5,7.3 \mathrm{~Hz}, 4.3 \mathrm{H}), 0.91(\mathrm{dt}, J=11.3,7.3 \mathrm{~Hz}, 7.3 \mathrm{H})$. 
${ }^{13} \mathrm{C}$ NMR $\left(101 \mathrm{MHz}, \mathrm{CDCl}_{3}\right) \delta 170.6,170.1,139.6,137.2,137.1,135.5,134.1,133.76,132.6$, 131.5, 130.7, 130.5, 129.1, 127.8, 127.2, 126.7, 126.4, 125.9, 48.6, 48.1, 48.0, 45.4, 32.8, 31.5, $30.7,29.6,25.9,25.8,24.6,24.5,22.6,22.1,19.6,19.6,19.3,19.1,17.1,13.9$.

HRMS (ESI-TOF) m/z: [M + Na] ${ }^{+}$Calcd for $\mathrm{C}_{19} \mathrm{H}_{27} \mathrm{NONa} 308.1985$; Found 308.1995.

(E)-(3-(Hex-1-en-1-yl)naphthalen-2-yl)(pyrrolidin-1-yl)methanone/(E)-(3-(Hex-2-en-2yl)naphthalen-2-yl)(pyrrolidin-1-yl)methanone (30a/3oa').<smiles>CCCC/C=C/c1cc2ccccc2cc1C(=O)N1CCCC1</smiles><smiles>CCC/C=C(\C)c1cc2ccccc2cc1C(=O)N1CCCC1</smiles>

Pale yellow liquid; eluent (hexane, $10 \%$ ethyl acetate). The reaction scale is $50 \mathrm{mg}, 59 \mathrm{mg}$ of product was isolated and yield is $86 \%$.

${ }^{1} \mathrm{H}$ NMR $\left(400 \mathrm{MHz}, \mathrm{CDCl}_{3}\right) \delta 7.90(\mathrm{~s}, 1 \mathrm{H}), 7.81-7.74(\mathrm{~m}, 5 \mathrm{H}), 7.72(\mathrm{~s}, 1 \mathrm{H}), 7.69(\mathrm{~s}, 1 \mathrm{H})$, $7.43(\mathrm{dd}, J=15.3,7.3 \mathrm{~Hz}, 4 \mathrm{H}), 6.53(\mathrm{~d}, J=15.7 \mathrm{~Hz}, 1 \mathrm{H}), 6.38-6.28(\mathrm{~m}, 0.9 \mathrm{H}), 5.65(\mathrm{t}, J=$ $7.2 \mathrm{~Hz}, 0.5 \mathrm{H}), 3.70(\mathrm{t}, J=7.0 \mathrm{~Hz}, 2.7 \mathrm{H}), 3.62(\mathrm{t}, J=6.8 \mathrm{~Hz}, 1.3 \mathrm{H}), 3.18(\mathrm{~d}, J=6.4 \mathrm{~Hz}, 1 \mathrm{H})$, $3.11(\mathrm{q}, J=6.7 \mathrm{~Hz}, 3.3 \mathrm{H}), 2.24(\mathrm{dd}, J=14.1,7.0 \mathrm{~Hz}, 2.2 \mathrm{H}), 2.16(\mathrm{dd}, J=14.5,7.2 \mathrm{~Hz}, 1.4 \mathrm{H})$, $2.08(\mathrm{~s}, 1.8 \mathrm{H}), 1.93(\mathrm{dt}, J=14.0,6.9 \mathrm{~Hz}, 4.6 \mathrm{H}), 1.81(\mathrm{td}, J=13.0,6.4 \mathrm{~Hz}, 4.6 \mathrm{H}), 1.45(\mathrm{dd}, J=$ $14.8,7.3 \mathrm{~Hz}, 3.8 \mathrm{H}), 1.37$ (dd, $J=14.5,7.2 \mathrm{~Hz}, 3 \mathrm{H}), 0.95(\mathrm{dt}, J=14.7,7.2 \mathrm{~Hz}, 5.5 \mathrm{H})$.

${ }^{13} \mathrm{C}$ NMR $\left(101 \mathrm{MHz}, \mathrm{CDCl}_{3}\right) \delta 170.1,169.6,140.1,135.1,135.0,134.4,134.3,133.4,133.3$, 132.5, 132.1, 132.0, 131.7, 131.4, 127.7, 127.6, 127.6, 126.7, 126.7, 126.2, 126.0, 125.4, 124.8, $48.9,48.2,45.6,45.5,32.9,31.4,30.8,29.6,25.9,24.6,24.5,22.7,22.2,17.4,13.9,13.9$.

HRMS (ESI-TOF) m/z: [M + Na] ${ }^{+}$Calcd for $\mathrm{C}_{21} \mathrm{H}_{25} \mathrm{NONa} 330.1834$; Found 330.1838.

(E)-(2-(Hex-1-en-1-yl)-3,5-dimethoxyphenyl)(pyrrolidin-1-yl)methanone/(E)-(2-(Hex-2en-1-yl)-3,5-dimethoxyphenyl)(pyrrolidin-1-yl)methanone (3pa/3pa')<smiles>CCC/C=C/Cc1c(OC)cc(OC)cc1C(=O)N1CCCC1</smiles>

Pale yellow liquid; eluent (hexane, $20 \%$ ethyl acetate). The reaction scale is $50 \mathrm{mg}, 40 \mathrm{mg}$ of product was isolated and yield is $60 \%$.

${ }^{1} \mathrm{H}$ NMR $\left(400 \mathrm{MHz}, \mathrm{CDCl}_{3}\right) \delta 6.42(\mathrm{~d}, J=3.4 \mathrm{~Hz}, 2.6 \mathrm{H}), 6.38(\mathrm{~s}, 1.4 \mathrm{H}), 6.31(\mathrm{~s}, 1 \mathrm{H}), 6.09(\mathrm{dd}$, $J=15.3,7.5 \mathrm{~Hz}, 1 \mathrm{H}), 5.36(\mathrm{dd}, J=14.5,6.8 \mathrm{~Hz}, 1 \mathrm{H}), 3.81-3.78(\mathrm{~m}, 8.8 \mathrm{H}), 3.65-3.58(\mathrm{~m}$, $3.1 \mathrm{H}), 3.25(\mathrm{~d}, J=6.4 \mathrm{~Hz}, 1.1 \mathrm{H}), 3.15(\mathrm{~d}, J=7.1 \mathrm{~Hz}, 2 \mathrm{H}), 2.18-2.09(\mathrm{~m}, 2.4 \mathrm{H}), 1.91(\mathrm{t}, J=$ 
$7.2 \mathrm{~Hz}, 6.7 \mathrm{H}), 1.80(\mathrm{t}, J=6.9 \mathrm{~Hz}, 6 \mathrm{H}), 1.41-1.29(\mathrm{~m}, 6.3 \mathrm{H}), 0.92-0.87(\mathrm{~m}, 3.4 \mathrm{H}), 0.84(\mathrm{~s}$, $1.9 \mathrm{H})$.

${ }^{13} \mathrm{C}$ NMR $\left(101 \mathrm{MHz}, \mathrm{CDCl}_{3}\right) \delta 169.8,169.5,159.5,159.1,158.8,158.1,139.1,138.1,136.6$, 135.9, 134.8, 132.7, 130.8, 130.4, 129.4, 128.1, 128.0, 121.8, 120.9, 118.0, 116.6, 102.2, 101.4, 99.1, 98.9, 55.6, 55.5, 55.4, 48.5, 47.6, 45.6, 45.3, 38.7, 36.4, 34.6, 33.7, 31.7, 29.9, 29.6, 29.28, $27.9,25.8,24.6,24.5,22.6,22.3,13.9,13.8,13.7$.

HRMS (ESI-TOF) m/z: [M + Na $]^{+}$Calcd for $\mathrm{C}_{19} \mathrm{H}_{27} \mathrm{NO}_{3} \mathrm{Na} 340.1889$; Found 340.1895.

(E)-(2-(Hex-1-en-1-yl)-3,4,5-trimethoxyphenyl)(pyrrolidin-1-yl)methanone/(E)-(2-(Hex2-en-1-yl)-3,4,5-trimethoxyphenyl)(pyrrolidin-1-yl)methanone (3qa/3qa').<smiles>CCC/C=C/Cc1c(C(=O)N2CCCC2)cc(OC)c(OC)c1OC</smiles>

Pale yellow liquid; eluent (hexane, $25 \%$ ethyl acetate). The reaction scale is $50 \mathrm{mg}, 41 \mathrm{mg}$ of product was isolated and yield is $63 \%$.

${ }^{1} \mathrm{H}$ NMR $\left(400 \mathrm{MHz}, \mathrm{CDCl}_{3}\right) \delta 6.59(\mathrm{~s}, 1 \mathrm{H}), 6.51(\mathrm{~s}, 0.4 \mathrm{H}), 6.40(\mathrm{~d}, J=16.0 \mathrm{~Hz}, 1 \mathrm{H}), 6.10(\mathrm{dd}$, $J=15.0,7.8 \mathrm{~Hz}, 1 \mathrm{H}), 5.44(\mathrm{~s}, 0.5 \mathrm{H}), 3.86(\mathrm{~s}, 5.5 \mathrm{H}), 3.84(\mathrm{~s}, 3.4 \mathrm{H}), 3.81(\mathrm{~s}, 1.6 \mathrm{H}), 3.79(\mathrm{~s}, 3 \mathrm{H})$, $3.60(\mathrm{dd}, J=14.9,7.1 \mathrm{~Hz}, 3 \mathrm{H}), 3.29(\mathrm{~d}, J=4.8 \mathrm{~Hz}, 0.6 \mathrm{H}), 3.15(\mathrm{t}, J=6.5 \mathrm{~Hz}, 1 \mathrm{H}), 3.07(\mathrm{~s}$, $2 \mathrm{H}), 2.15(\mathrm{~d}, J=6.6 \mathrm{~Hz}, 2 \mathrm{H}), 1.90(\mathrm{~s}, 3.5 \mathrm{H}), 1.82(\mathrm{~d}, J=5.7 \mathrm{~Hz}, 3.5 \mathrm{H}), 1.73-1.55(\mathrm{~m}, 2.3 \mathrm{H})$, $1.36(\mathrm{~s}, 5.7 \mathrm{H}), 0.90(\mathrm{t}, J=6.9 \mathrm{~Hz}, 3.7 \mathrm{H}), 0.84(\mathrm{~d}, J=7.1 \mathrm{~Hz}, 1.4 \mathrm{H})$.

${ }^{13} \mathrm{C}$ NMR (101 MHz, $\left.\mathrm{CDCl}_{3}\right) \delta 168.6,168.3,151.4,150.9,150.4,141.6,134.6,132.1,130.9$, 129.9, 128.7, 127.4, 127.2, 122.6, 120.8, 104.8, 104.0, 60.0, 59.9, 59.8, 59.7, 55.1, 55.0, 47.7, 46.7, 44.6, 44.4, 37.7, 35.4, 33.6, 32.6, 30.6, 29.3, 28.6, 26.8, 24.8, 23.6, 23.5, 21.6, 21.2 12.9, 12.7 .

HRMS (ESI-TOF) m/z: [M + Na] ${ }^{+}$Calcd for $\mathrm{C}_{20} \mathrm{H}_{29} \mathrm{NO}_{4} \mathrm{Na}$ 370.1994; Found 370.2000.

(E)-(2-(Hex-1-en-1-yl)-3,4,6-trimethoxyphenyl)(pyrrolidin-1-yl)methanone (3ra).<smiles>CCCC/C=C/c1c(OC)c(OC)cc(OC)c1C(=O)N1CCCC1</smiles>

Pale yellow liquid; eluent (hexane, $25 \%$ ethyl acetate). The reaction scale is $50 \mathrm{mg}, 30 \mathrm{mg}$ of product was isolated and yield is $46 \%$. 
${ }^{1} \mathrm{H}$ NMR $\left(400 \mathrm{MHz}, \mathrm{CDCl}_{3}\right) \delta 7.20(\mathrm{~s}, 1 \mathrm{H}), 6.33(\mathrm{~d}, J=9.8 \mathrm{~Hz}, 1 \mathrm{H}), 6.30-6.19(\mathrm{~m}, 1 \mathrm{H}), 3.81$ $(\mathrm{s}, 3 \mathrm{H}), 3.73(\mathrm{~s}, 3 \mathrm{H}), 3.61(\mathrm{~s}, 3 \mathrm{H}), 2.98(\mathrm{t}, J=6.3 \mathrm{~Hz}, 2 \mathrm{H}), 2.14-2.05(\mathrm{~m}, 2 \mathrm{H}), 1.91-1.66$ $(\mathrm{m}, 6 \mathrm{H}), 1.33-1.24(\mathrm{~m}, 4 \mathrm{H}), 0.83(\mathrm{t}, J=7.0 \mathrm{~Hz}, 3 \mathrm{H})$.

${ }^{13} \mathrm{C}$ NMR $\left(101 \mathrm{MHz}, \mathrm{CDCl}_{3}\right) \delta 167.2,153.3,152.0,140.8,137.4,129.4,122.1,118.5,95.9$, $60.4,56.4,56.0,47.3,45.3,33.7,31.5,25.7,24.7,22.2,13.9$.

HRMS (ESI-TOF) m/z: [M + Na $]^{+}$Calcd for $\mathrm{C}_{20} \mathrm{H}_{29} \mathrm{NO}_{4} \mathrm{Na} 370.1994$; Found 370.1999.

(E)-2-(Hex-1-en-1-yl)-4-methylbenzamide (3sa).<smiles>CCCC/C=C/c1cc(C)ccc1C(N)=O</smiles>

White solid; eluent (hexane, $10 \%$ ethyl acetate). The reaction scale is $50 \mathrm{mg}, 34 \mathrm{mg}$ of product was isolated and yield is $40 \%$.

${ }^{1} \mathrm{H}$ NMR (500 MHz, CDCl $) \delta 7.46(\mathrm{~d}, \mathrm{~J}=7.8 \mathrm{~Hz}, 1 \mathrm{H}), 7.30(\mathrm{~s}, 1 \mathrm{H}), 7.07$ (d, J = 7.5 Hz, 1H), $6.79(\mathrm{~d}, \mathrm{~J}=15.7 \mathrm{~Hz}, 1 \mathrm{H}), 6.18(\mathrm{dt}, \mathrm{J}=15.8,6.9 \mathrm{~Hz}, 1 \mathrm{H}), 5.82(\mathrm{~s}, 2 \mathrm{H}), 2.37(\mathrm{~s}, 3 \mathrm{H}), 2.24(\mathrm{q}, \mathrm{J}$ $=7.2 \mathrm{~Hz}, 2 \mathrm{H}), 1.50-1.43(\mathrm{~m}, 2 \mathrm{H}), 1.41-1.36(\mathrm{~m}, 2 \mathrm{H}), 0.93(\mathrm{t}, \mathrm{J}=7.3 \mathrm{~Hz}, 3 \mathrm{H})$.

${ }^{13} \mathrm{C} \mathrm{NMR}\left(126 \mathrm{MHz}, \mathrm{CDCl}_{3}\right) \delta 171.4,140.7,136.4,134.5,130.6,128.0,127.62$ 127.5, 127.4, $32.8,31.4,22.3,21.4,13.9$.

HRMS (ESI-TOF) m/z: [M + Na $]^{+}$Calcd for $\mathrm{C}_{14} \mathrm{H}_{19} \mathrm{NONa} 240.1364$; Found 240.1367.

(E)-4-Bromo-2-(hex-1-en-1-yl)benzamide (3ta).<smiles>CCCC/C=C/c1cc(Br)ccc1C(N)=O</smiles>

White solid; eluent (hexane, 10\% ethyl acetate). The reaction scale is $50 \mathrm{mg}, 28 \mathrm{mg}$ of product was isolated and yield is $42 \%$.

${ }^{1} \mathrm{H}$ NMR $\left(500 \mathrm{MHz}, \mathrm{CDCl}_{3}\right) \delta 7.64(\mathrm{~d}, \mathrm{~J}=1.9 \mathrm{~Hz}, 1 \mathrm{H}), 7.41-7.37(\mathrm{~m}, 2 \mathrm{H}), 6.72(\mathrm{~d}, \mathrm{~J}=15.7$ $\mathrm{Hz}, 1 \mathrm{H}), 6.22(\mathrm{dt}, \mathrm{J}=15.6,6.9 \mathrm{~Hz}, 1 \mathrm{H}), 6.02(\mathrm{~s}, 1 \mathrm{H}), 5.82(\mathrm{~s}, 1 \mathrm{H}), 2.24(\mathrm{q}, \mathrm{J}=7.2 \mathrm{~Hz}, 2 \mathrm{H})$, $1.50-1.44(\mathrm{~m}, 2 \mathrm{H}), 1.38$ (p, J = 7.3 Hz, 2H), 0.93 (t, J = 7.3 Hz, 3H).

${ }^{13} \mathrm{C} \mathrm{NMR}\left(126 \mathrm{MHz}, \mathrm{CDCl}_{3}\right) \delta 170.5,138.4,136.2,132.2,129.8,129.6,129.3,126.1,125.0$, $32.8,31.2,22.3,13.9$.

HRMS (ESI-TOF) m/z: [M + Na $]^{+}$Calcd for $\mathrm{C}_{13} \mathrm{H}_{16} \mathrm{BrNONa} 304.0313$; Found 304.0317.

(E)-2-(Hex-1-en-1-yl)-N,4-dimethylbenzamide/2-(Hex-1-en-2-yl)-N,4dimethylbenzamide (3ua/3ua") 
Pale yellow liquid; eluent (hexane, $10 \%$ ethyl acetate). The reaction scale is $50 \mathrm{mg}, 39 \mathrm{mg}$ of product was isolated and yield is $50 \%$.<smiles>C=C(CCCC)c1cc(C)ccc1C(=O)NC</smiles>

${ }^{1} \mathrm{H}$ NMR $\left(400 \mathrm{MHz}, \mathrm{CDCl}_{3}\right) \delta 7.33(\mathrm{~d}, \mathrm{~J}=7.8 \mathrm{~Hz}, 1 \mathrm{H}), 7.20(\mathrm{~d}, \mathrm{~J}=8 \mathrm{H}, 0.4 \mathrm{H}), 7.02(\mathrm{~d}, \mathrm{~J}=6.8$ $\mathrm{Hz}, 2 \mathrm{H}), 7.00-6.94(\mathrm{~m}, 1 \mathrm{H}), 6.66(\mathrm{~d}, \mathrm{~J}=15.7 \mathrm{~Hz}, 1 \mathrm{H}), 6.15$ (dt, J = 14.9, $6.9 \mathrm{~Hz}, 1 \mathrm{H}), 5.86$ $(\mathrm{s}, 1 \mathrm{H}), 5.20(\mathrm{~s}, 0.1 \mathrm{H}), 5.07(\mathrm{~s}, 0.1 \mathrm{H}), 2.96(\mathrm{t}, \mathrm{J}=3.0 \mathrm{~Hz}, 4.5 \mathrm{H}), 2.75-2.69(\mathrm{~m}, 1 \mathrm{H}), 2.34(\mathrm{~s}$, $3 \mathrm{H}), 2.33$ (s, 2H), 2.22 (d, J = 7.2 Hz, 2H), $1.49-1.42(\mathrm{~m}, 2.8 \mathrm{H}), 1.37$ (q, J = 7.5 Hz, 3.7H), $0.93(\mathrm{t}, \mathrm{J}=7.2 \mathrm{~Hz}, 4.8 \mathrm{H})$.

${ }^{13} \mathrm{C}$ NMR $\left(101 \mathrm{MHz}, \mathrm{CDCl}_{3}\right) \delta 171.1,170.4,141.1,139.9,139.6,135.9,134.0,133.5,132.0$, $131.9,131.0,130.9,130.7,130.1,129.8,129.3,128.9,127.7,127.7,127.6,127.5,127.4,127.3$, 127.0, 126.8, 126.2, 38.6, 36.5, 36.4, 34.6, 33.2, 32.8, 31.9, 31.7, 31.6, 31.4, 30.5, 29.6, 29.3, $29.3,27.8,26.7,26.6,26.6,22.6,22.5,22.2,21.3,21.2,14.0,13.9,13.8,13.6$.

HRMS (ESI-TOF) m/z: [M + Na $]^{+}$Calcd for $\mathrm{C}_{15} \mathrm{H}_{21} \mathrm{NONa} 254.1521$; Found 254.1524.

(E)-(2-(3,3-Dimethylbut-1-en-1-yl)-4-methoxyphenyl)(pyrrolidin-1-yl)methanone (4ab).<smiles>COc1ccc(C(=O)N2CCCC2)c(/C=C/C(C)(C)C)c1</smiles>

White solid; eluent (hexane, $15 \%$ ethyl acetate). The reaction scale is $50 \mathrm{mg}, 51 \mathrm{mg}$ of product was isolated and yield is $72 \%$.

${ }^{1} \mathrm{H}$ NMR (400 MHz, $\left.\mathrm{CDCl}_{3}\right) \delta 7.18(\mathrm{~d}, \mathrm{~J}=8.4 \mathrm{~Hz}, 1 \mathrm{H}), 6.94(\mathrm{~s}, 1 \mathrm{H}), 6.77(\mathrm{~d}, \mathrm{~J}=8.4 \mathrm{~Hz}, 1 \mathrm{H})$, $6.31(\mathrm{~d}, \mathrm{~J}=16.1 \mathrm{~Hz}, 1 \mathrm{H}), 6.20(\mathrm{~d}, \mathrm{~J}=16.1 \mathrm{~Hz}, 1 \mathrm{H}), 3.81(\mathrm{~s}, 3 \mathrm{H}), 3.62(\mathrm{t}, \mathrm{J}=6.9 \mathrm{~Hz}, 2 \mathrm{H}), 3.08$ $(\mathrm{t}, \mathrm{J}=6.7 \mathrm{~Hz}, 2 \mathrm{H}), 1.93-1.87(\mathrm{~m}, 2 \mathrm{H}), 1.83-1.78(\mathrm{~m}, 2 \mathrm{H}), 1.07$ (s, 9H).

${ }^{13} \mathrm{C} \mathrm{NMR}\left(101 \mathrm{MHz}, \mathrm{CDCl}_{3}\right) \delta 169.8,159.9,144.7,136.0,129.3,127.8,121.8,112.7,110.7$, 55.3, 48.0, 45.5, 33.5, 29.4, 25.9, 24.6.

HRMS (ESI-TOF) m/z: [M + Na $]^{+}$Calcd for $\mathrm{C}_{18} \mathrm{H}_{25} \mathrm{NO}_{2} \mathrm{Na} 310.1783$; Found 310.1790 .

(E)-(2-(3,3-Dimethylbut-1-en-1-yl)-4-methylphenyl)(pyrrolidin-1-yl)methanone (4bb).<smiles>Cc1ccc(C(=O)N2CCCC2)c(/C=C/C(C)(C)C)c1</smiles> 
Pale yellow liquid; eluent (hexane, $12 \%$ ethyl acetate). The reaction scale is $50 \mathrm{mg}, 41 \mathrm{mg}$ of product was isolated and yield is $57 \%$.

${ }^{1} \mathrm{H}$ NMR $\left(500 \mathrm{MHz}, \mathrm{CDCl}_{3}\right) \delta 7.20(\mathrm{~s}, 1 \mathrm{H}), 7.07(\mathrm{~d}, J=7.7 \mathrm{~Hz}, 1 \mathrm{H}), 6.97(\mathrm{~d}, J=7.6 \mathrm{~Hz}, 1 \mathrm{H})$, $6.24(\mathrm{~d}, J=16.1 \mathrm{~Hz}, 1 \mathrm{H}), 6.14(\mathrm{~d}, J=16.1 \mathrm{~Hz}, 1 \mathrm{H}), 3.57(\mathrm{t}, J=7.0 \mathrm{~Hz}, 2 \mathrm{H}), 3.01(\mathrm{t}, J=6.7$ $\mathrm{Hz}, 2 \mathrm{H}), 2.27(\mathrm{~s}, 3 \mathrm{H}), 1.86(\mathrm{dd}, J=13.8,6.9 \mathrm{~Hz}, 2 \mathrm{H}), 1.75(\mathrm{dd}, J=13.4,6.7 \mathrm{~Hz}, 2 \mathrm{H}), 1.01$ (s, 9H).

${ }^{13} \mathrm{C}$ NMR $\left(126 \mathrm{MHz}, \mathrm{CDCl}_{3}\right) \delta 170.1,144.3,138.6,134.1,133.7,127.7,126.3,126.2,121.7$, $47.9,45.5,33.5,29.5,25.9,24.6,21.3$.

HRMS (ESI-TOF) m/z: [M + Na] ${ }^{+}$Calcd for $\mathrm{C}_{18} \mathrm{H}_{25} \mathrm{NONa} 294.1834$; Found 294.1835.

(E)-(2-(3,3-Dimethylbut-1-en-1-yl)-4-fluorophenyl)(pyrrolidin-1-yl)methanone (4cb).<smiles>CC(C)(C)/C=C/c1cc(F)ccc1C(=O)N1CCCC1</smiles>

Pale yellow liquid; eluent (hexane, $12 \%$ ethyl acetate). The reaction scale is $50 \mathrm{mg}, 31 \mathrm{mg}$ of product was isolated and yield is $44 \%$.

${ }^{1} \mathrm{H}$ NMR $\left(500 \mathrm{MHz}, \mathrm{CDCl}_{3}\right) \delta 7.21(\mathrm{dd}, J=7.8,6.4 \mathrm{~Hz}, 1 \mathrm{H}), 7.14(\mathrm{~d}, J=10.0 \mathrm{~Hz}, 1 \mathrm{H}), 6.91$ $(\mathrm{t}, J=8.3 \mathrm{~Hz}, 1 \mathrm{H}), 6.28(\mathrm{~d}, J=16.1 \mathrm{~Hz}, 1 \mathrm{H}), 6.23(\mathrm{~d}, J=16.1 \mathrm{~Hz}, 1 \mathrm{H}), 3.63(\mathrm{t}, J=7.0 \mathrm{~Hz}$, $2 \mathrm{H}), 3.06(\mathrm{t}, J=6.7 \mathrm{~Hz}, 2 \mathrm{H}), 1.94-1.89(\mathrm{~m}, 2 \mathrm{H}), 1.84-1.80(\mathrm{~m}, 2 \mathrm{H}), 1.07(\mathrm{~s}, 9 \mathrm{H})$.

${ }^{13} \mathrm{C} \mathrm{NMR}\left(126 \mathrm{MHz}, \mathrm{CDCl}_{3}\right) \delta 169.0,163.9,161.9,145.8,137.0,136.9,132.5,128.2,128.1$, $120.9,114.0,113.8,112.3,112.1,48.0,45.6,33.6,29.3,25.9,24.6$.

HRMS (ESI-TOF) m/z: [M + Na $]^{+}$Calcd for $\mathrm{C}_{17} \mathrm{H}_{22} \mathrm{FNONa}$ 298.1583; Found 298.1586.

(E)-4-Bromo-2-(3,3-dimethylbut-1-en-1-yl)benzamide (4eb).<smiles>CC(C)(C)/C=C/c1cc(Br)ccc1C(N)=O</smiles>

White solid; eluent (hexane, $12 \%$ ethyl acetate). The reaction scale is $50 \mathrm{mg}, 33 \mathrm{mg}$ of product was isolated and yield is $50 \%$.

${ }^{1} \mathrm{H}$ NMR $\left(400 \mathrm{MHz}, \mathrm{CDCl}_{3}\right) \delta 7.63(\mathrm{~s}, 1 \mathrm{H}), 7.42(\mathrm{~d}, \mathrm{~J}=8.2 \mathrm{~Hz}, 1 \mathrm{H}), 7.38(\mathrm{~d}, \mathrm{~J}=8.3 \mathrm{~Hz}, 1 \mathrm{H})$, $6.66(\mathrm{~d}, \mathrm{~J}=16.1 \mathrm{~Hz}, 1 \mathrm{H}), 6.22(\mathrm{~d}, \mathrm{~J}=16.0 \mathrm{~Hz}, 1 \mathrm{H}), 6.03(\mathrm{~s}, 1 \mathrm{H}), 5.82(\mathrm{~s}, 1 \mathrm{H}), 1.12(\mathrm{~s}, 9 \mathrm{H})$. 
${ }^{13} \mathrm{C} \mathrm{NMR}\left(101 \mathrm{MHz}, \mathrm{CDCl}_{3}\right) \delta 170.5,146.6,138.5,132.4,129.8,129.6,129.5,125.0,121.5$, 33.9, 29.4 .

HRMS (ESI-TOF) m/z: [M + Na $]^{+}$Calcd for $\mathrm{C}_{13} \mathrm{H}_{16} \mathrm{BrNONa}$ 304.0313; Found 304.0303.

(E)-(2-(2-Cyclohexylvinyl)-4-methylphenyl)(pyrrolidin-1-yl)methanone (4bc).<smiles>Cc1ccc(C(=O)N2CCCC2)c(/C=C/C2CCCCC2)c1</smiles>

Pale yellow liquid; eluent (hexane, 12\% ethyl acetate). The reaction scale is $50 \mathrm{mg}, 72 \mathrm{mg}$ of product was isolated and yield is $92 \%$.

${ }^{1} \mathrm{H} \mathrm{NMR}\left(400 \mathrm{MHz}, \mathrm{CDCl}_{3}\right) \delta 7.27(\mathrm{~s}, 1 \mathrm{H}), 7.12(\mathrm{~d}, J=7.6 \mathrm{~Hz}, 1 \mathrm{H}), 7.02(\mathrm{~d}, J=7.6 \mathrm{~Hz}, 1 \mathrm{H})$, $6.34(\mathrm{~d}, J=15.9 \mathrm{~Hz}, 1 \mathrm{H}), 6.13(\mathrm{dd}, J=15.9,7.1 \mathrm{~Hz}, 1 \mathrm{H}), 3.64(\mathrm{t}, J=6.7 \mathrm{~Hz}, 2 \mathrm{H}), 3.09(\mathrm{t}, J=$ $6.5 \mathrm{~Hz}, 2 \mathrm{H}), 2.33(\mathrm{~s}, 3 \mathrm{H}), 2.13-2.06(\mathrm{~m}, 1 \mathrm{H}), 1.95-1.89(\mathrm{~m}, 2 \mathrm{H}), 1.82(\mathrm{dd}, J=12.8,6.3 \mathrm{~Hz}$, 2H), $1.74(\mathrm{~d}, J=10.6 \mathrm{~Hz}, 4 \mathrm{H}), 1.29(\mathrm{~d}, J=12.6 \mathrm{~Hz}, 2 \mathrm{H}), 1.25-1.16(\mathrm{~m}, 2 \mathrm{H}), 1.14(\mathrm{~d}, J=12.0$ $\mathrm{Hz}, 2 \mathrm{H})$.

${ }^{13} \mathrm{C}$ NMR $\left(126 \mathrm{MHz}, \mathrm{CDCl}_{3}\right) \delta 170.1,139.3,138.6,134.1,130.0,129.0,127.6,126.2,126.2$, 124.2, 48.1, 45.5, 41.3, 32.9, 26.1, 25.9, 25.9, 24.6, 21.3.

HRMS (ESI-TOF) m/z: [M + Na $]^{+}$Calcd for $\mathrm{C}_{20} \mathrm{H}_{27} \mathrm{NONa} 320.1994$; Found 320.1994 .

(E)-(3-(2-Cyclohexylvinyl)thiophen-2-yl)(pyrrolidin-1-yl)methanone (4vc).

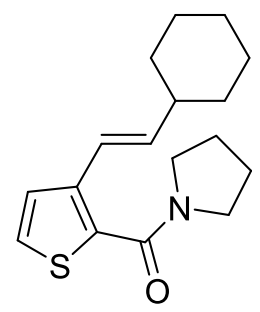

Pale yellow liquid; eluent (hexane, $8 \%$ ethyl acetate). The reaction scale is $50 \mathrm{mg}, 26 \mathrm{mg}$ of product was isolated and yield is $33 \%$.

${ }^{1} \mathrm{H}$ NMR $\left(400 \mathrm{MHz}, \mathrm{CDCl}_{3}\right) \delta 7.23(\mathrm{~d}, \mathrm{~J}=5.1 \mathrm{~Hz}, 1 \mathrm{H}), 7.13(\mathrm{~d}, \mathrm{~J}=5.2 \mathrm{~Hz}, 1 \mathrm{H}), 6.44(\mathrm{~d}, \mathrm{~J}=$ $16.0 \mathrm{~Hz}, 1 \mathrm{H}), 6.07(\mathrm{dd}, \mathrm{J}=16.0,7.1 \mathrm{~Hz}, 1 \mathrm{H}), 3.63(\mathrm{~s}, 2 \mathrm{H}), 3.47-3.36(\mathrm{~m}, 2 \mathrm{H}), 2.09$ (dt, J = 11.6, 3.9 Hz, 1H), $1.73(\mathrm{~d}, \mathrm{~J}=13.6 \mathrm{~Hz}, 6 \mathrm{H}), 1.68-1.62(\mathrm{~m}, 2 \mathrm{H}), 1.29(\mathrm{~d}, \mathrm{~J}=11.0 \mathrm{~Hz}, 2 \mathrm{H})$, $1.15(\mathrm{dd}, \mathrm{J}=14.0,11.0 \mathrm{~Hz}, 4 \mathrm{H})$.

${ }^{13} \mathrm{C}$ NMR $\left(101 \mathrm{MHz}, \mathrm{CDCl}_{3}\right) \delta 163.9,139.4,139.3,131.1,125.7,125.1,120.5,41.21,38.6$, $36.4,32.8,27.8,26.4,26.0,25.9$.

HRMS (ESI-TOF) m/z: [M + Na ${ }^{+}$Calcd for $\mathrm{C}_{17} \mathrm{H}_{23} \mathrm{NOSNa}$ 312.1398; Found 312.1401. 
(E)-(2-(2-Cyclopentylvinyl)-4-methoxyphenyl)(pyrrolidin-1-yl)methanone (4ad).<smiles>COc1ccc(C(=O)N2CCCC2)c(/C=C/C2CCCC2)c1</smiles>

Pale yellow liquid; eluent (hexane, $15 \%$ ethyl acetate). The reaction scale is $50 \mathrm{mg}, 34 \mathrm{mg}$ of product was isolated and yield is $46 \%$.

${ }^{1} \mathrm{H}$ NMR $\left(400 \mathrm{MHz}, \mathrm{CDCl}_{3}\right) \delta 7.17(\mathrm{~d}, \mathrm{~J}=8.5 \mathrm{~Hz}, 1 \mathrm{H}), 6.97(\mathrm{~d}, \mathrm{~J}=2.6 \mathrm{~Hz}, 1 \mathrm{H}), 6.77(\mathrm{dd}, \mathrm{J}=$ 8.5, 2.5 Hz, 1H), 6.38 (d, J = 15.7 Hz, 1H), 6.17 (dd, J = 15.7, 7.9 Hz, 1H), $3.82(\mathrm{~s}, 3 \mathrm{H}), 3.63$ $(\mathrm{t}, \mathrm{J}=7.1 \mathrm{~Hz}, 2 \mathrm{H}), 3.11(\mathrm{t}, \mathrm{J}=6.7 \mathrm{~Hz}, 2 \mathrm{H}), 2.61-2.53(\mathrm{~m}, 1 \mathrm{H}), 1.96-1.90(\mathrm{~m}, 2 \mathrm{H}), 1.86-$ $1.79(\mathrm{~m}, 4 \mathrm{H}), 1.72-1.65(\mathrm{~m}, 4 \mathrm{H}), 1.40-1.34(\mathrm{~m}, 2 \mathrm{H})$.

${ }^{13} \mathrm{C}$ NMR $\left(101 \mathrm{MHz}, \mathrm{CDCl}_{3}\right) \delta 169.8,159.8,138.6,135.9,129.2,127.8,124.8,112.7,110.6$, $55.3,48.2,45.5,43.9,33.2,25.9,25.2,24.6$.

HRMS (ESI-TOF) m/z: [M + Na] ${ }^{+}$Calcd for $\mathrm{C}_{19} \mathrm{H}_{25} \mathrm{NO}_{2} \mathrm{Na}$ 322.1783; Found 322.1790.

(E)-(3-(Oct-1-en-1-yl)naphthalen-2-yl)(pyrrolidin-1-yl)methanone/(E)-(3-(Oct-2-en-2yl)naphthalen-2-yl)(pyrrolidin-1-yl)methanone (4oe/4oe').<smiles>CCCCCC/C=C/c1cc2ccccc2cc1C(=O)N1CCCC1</smiles>

Pale yellow liquid; eluent (hexane, $10 \%$ ethyl acetate). The reaction scale is $50 \mathrm{mg}, 48 \mathrm{mg}$ of product was isolated and yield is $65 \%$.

${ }^{1} \mathrm{H}$ NMR (400 MHz, $\left.\mathrm{CDCl}_{3}\right) \delta 7.90(\mathrm{~s}, 1 \mathrm{H}), 7.82-7.75(\mathrm{~m}, 8 \mathrm{H}), 7.72(\mathrm{~s}, 2 \mathrm{H}), 7.69(\mathrm{~s}, 2.4 \mathrm{H})$, $7.48(\mathrm{~d}, J=7.3 \mathrm{~Hz}, 2.8 \mathrm{H}), 7.44(\mathrm{~d}, J=8.2 \mathrm{~Hz}, 4.3 \mathrm{H}), 6.53(\mathrm{~d}, J=15.8 \mathrm{~Hz}, 1 \mathrm{H}), 6.35(\mathrm{dd}, J=$ $15.1,7.5 \mathrm{~Hz}, 1 \mathrm{H}), 5.66(\mathrm{t}, J=7.4 \mathrm{~Hz}, 0.9 \mathrm{H}), 3.71(\mathrm{t}, J=7.1 \mathrm{~Hz}, 4 \mathrm{H}), 3.66-3.60(\mathrm{~m}, 2.7 \mathrm{H})$, $3.18(\mathrm{~d}, J=6.8 \mathrm{~Hz}, 1.8 \mathrm{H}), 3.11(\mathrm{q}, J=6.2 \mathrm{~Hz}, 4.3 \mathrm{H}), 2.23(\mathrm{q}, J=7.6 \mathrm{~Hz}, 2.4 \mathrm{H}), 2.16(\mathrm{t}, J=$ $7.4 \mathrm{~Hz}, 2 \mathrm{H}), 2.08(\mathrm{~s}, 3 \mathrm{H}), 1.95(\mathrm{dt}, J=13.8,6.7 \mathrm{~Hz}, 8.4 \mathrm{H}), 1.82(\mathrm{dd}, J=13.1,6.6 \mathrm{~Hz}, 6.2 \mathrm{H})$, $1.45(\mathrm{dt}, J=12.4,6.7 \mathrm{~Hz}, 4.4 \mathrm{H}), 1.37-1.29(\mathrm{~m}, 12.3 \mathrm{H}), 0.90(\mathrm{t}, J=6.5 \mathrm{~Hz}, 6 \mathrm{H})$.

${ }^{13} \mathrm{C} \mathrm{NMR}\left(101 \mathrm{MHz}, \mathrm{CDCl}_{3}\right) \delta 170.2,169.6,140.1,135.1,135.1,134.4,134.2,133.4,133.3$, 132.2, 132.0, 131.7, 131.6, 127.8, 127.7, 127.6, 126.7, 126.7, 126.2, 126.0, 125.5, 124.8, 48.2, 45.6, 45.6, 33.3, 31.7, 31.6, 31.6, 29.6, 29.4, 29.3, 29.2, 28.9, 28.8, 26.1, 26.0, 25.9, 25.6, 24.6, $24.6,24.5,24.5,22.6,22.5,17.3,14.1,14.0,13.9$.

HRMS (ESI-TOF) m/z: $[\mathrm{M}+\mathrm{Na}]^{+}$Calcd for $\mathrm{C}_{23} \mathrm{H}_{29} \mathrm{NONa} 358.2147$; Found 358.2151. 
(E)-(3-(Dec-1-en-1-yl)naphthalen-2-yl)(pyrrolidin-1-yl)methanone/(E)-(3-(Dec-2-en-2yl)naphthalen-2-yl)(pyrrolidin-1-yl)methanone (4of/4of').

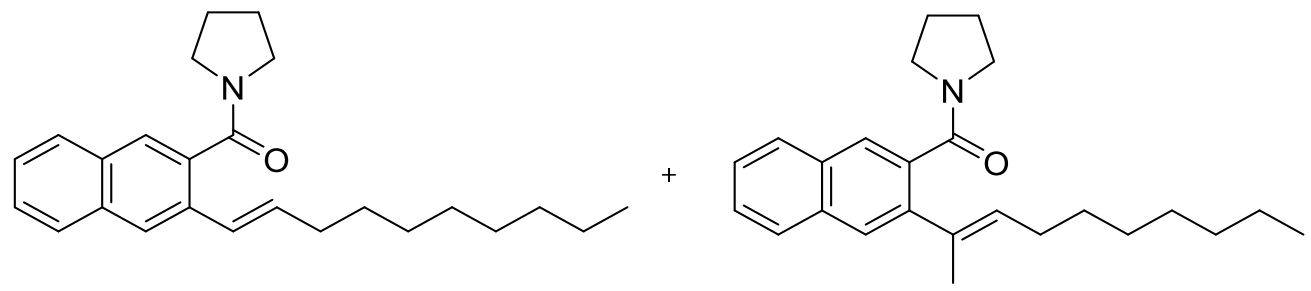

Pale yellow liquid; eluent (hexane, $10 \%$ ethyl acetate). The reaction scale is $50 \mathrm{mg}, 31 \mathrm{mg}$ of product was isolated and yield is $38 \%$.

${ }^{1} \mathrm{H}$ NMR $\left(400 \mathrm{MHz}, \mathrm{CDCl}_{3}\right) \delta 7.90(\mathrm{~s}, 1 \mathrm{H}), 7.79(\mathrm{t}, J=7.1 \mathrm{~Hz}, 8.9 \mathrm{H}), 7.72(\mathrm{~s}, 2.3 \mathrm{H}), 7.69$ (s, $2.9 \mathrm{H}), 7.45(\mathrm{p}, J=7.0 \mathrm{~Hz}, 7.8 \mathrm{H}), 6.53(\mathrm{~d}, J=15.9 \mathrm{~Hz}, 1 \mathrm{H}), 6.34(\mathrm{dt}, J=15.0,6.9 \mathrm{~Hz}, 1 \mathrm{H})$, $5.66(\mathrm{t}, J=7.3 \mathrm{~Hz}, 1 \mathrm{H}), 3.73-3.65(\mathrm{~m}, 5.3 \mathrm{H}), 3.63(\mathrm{t}, J=6.7 \mathrm{~Hz}, 3.1 \mathrm{H}), 3.24(\mathrm{t}, J=6.8 \mathrm{~Hz}$, $1 \mathrm{H}), 3.20-3.12(\mathrm{~m}, 4.4 \mathrm{H}), 3.10(\mathrm{~d}, J=6.4 \mathrm{~Hz}, 3.8 \mathrm{H}), 2.79(\mathrm{t}, J=7.9 \mathrm{~Hz}, 1 \mathrm{H}), 2.28-2.19(\mathrm{~m}$, 2.7H), $2.16(\mathrm{t}, J=7.3 \mathrm{~Hz}, 2.6 \mathrm{H}), 2.08(\mathrm{~s}, 3.7 \mathrm{H}), 1.96(\mathrm{dt}, J=13.7,6.5 \mathrm{~Hz}, 13.3 \mathrm{H}), 1.89-1.74$ $(\mathrm{m}, 10.2 \mathrm{H}), 1.45(\mathrm{dq}, J=18.9,6.9 \mathrm{~Hz}, 6.4 \mathrm{H}), 1.38-1.18(\mathrm{~m}, 33.7 \mathrm{H}), 0.88(\mathrm{dq}, J=7.8,4.7$, $4.1 \mathrm{~Hz}, 13.6 \mathrm{H})$.

${ }^{13} \mathrm{C}$ NMR (101 MHz, $\left.\mathrm{CDCl}_{3}\right) \delta 169.7,140.1,135.1,135.0,134.4,134.1,133.4,132.1,132.0$, 131.7, 131.4, 128.3, 127.9, 127.8, 127.7, 127.6, 127.3, 126.7, 126.7, 126.5, 126.2, 126.0, 125.6, $125.5,125.2$, 124.8, 48.9, 48.7, 48.2, 45.6, 45.6, 45.5, 45.4, 33.3, 31.9, 31.8, 29.6, 29.5, 29.5, $29.4,29.3,29.2,29.2,28.8,26.0,25.9,24.6,24.6,24.5,24.5,22.6,17.3,14.1,14.0$.

HRMS (ESI-TOF) m/z: [M + Na] ${ }^{+}$Calcd for $\mathrm{C}_{25} \mathrm{H}_{33} \mathrm{NONa}$ 386.2460; Found 386.2459.

(E)-(3,6-Dimethoxy-2-(oct-1-en-1-yl)phenyl)(pyrrolidin-1-yl)methanone (4we).<smiles>CCCCCC/C=C/c1c(OC)ccc(OC)c1C(=O)N1CCCC1</smiles>

Pale yellow liquid; eluent (hexane, 20\% ethyl acetate). The reaction scale is $50 \mathrm{mg}, 29 \mathrm{mg}$ of product was isolated and yield is $38 \%$.

${ }^{1} \mathrm{H}$ NMR $\left(400 \mathrm{MHz}, \mathrm{CDCl}_{3}\right) \delta 6.79(\mathrm{~d}, \mathrm{~J}=8.8 \mathrm{~Hz}, 1 \mathrm{H}), 6.72(\mathrm{~d}, \mathrm{~J}=9.1 \mathrm{~Hz}, 1 \mathrm{H}), 6.43-6.26$ $(\mathrm{m}, 2 \mathrm{H}), 3.78(\mathrm{~s}, 3 \mathrm{H}), 3.77(\mathrm{~s}, 3 \mathrm{H}), 3.69(\mathrm{q}, \mathrm{J}=7.0,6.6 \mathrm{~Hz}, 1 \mathrm{H}), 3.58-3.51(\mathrm{~m}, 1 \mathrm{H}), 3.07(\mathrm{t}$, $\mathrm{J}=6.3 \mathrm{~Hz}, 2 \mathrm{H}), 2.15(\mathrm{q}, \mathrm{J}=6.6 \mathrm{~Hz}, 2 \mathrm{H}), 1.87(\mathrm{ddd}, \mathrm{J}=23.7,13.6,5.7 \mathrm{~Hz}, 4 \mathrm{H}), 1.28(\mathrm{~s}, 8 \mathrm{H})$, $0.87(\mathrm{~d}, \mathrm{~J}=6.4 \mathrm{~Hz}, 3 \mathrm{H})$.

${ }^{13} \mathrm{C} \mathrm{NMR}\left(101 \mathrm{MHz}, \mathrm{CDCl}_{3}\right) \delta 167.1,151.6,149.6,137.1,127.1,124.7,122.2,111.6,109.9$, $56.3,56.2,47.2,45.2,34.1,31.7,29.4,28.9,25.8,24.6,22.6,14.1$.

HRMS (ESI-TOF) m/z: [M + Na $]^{+}$Calcd for $\mathrm{C}_{21} \mathrm{H}_{31} \mathrm{NO}_{3} \mathrm{Na}$ 368.2202; Found 368.2206. 


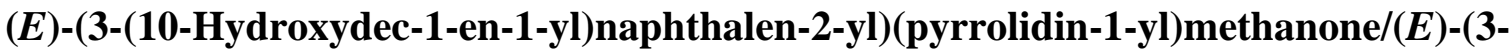
(10-Hydroxydec-2-en-2-yl)naphthalen-2-yl)(pyrrolidin-1-yl)methanone (5og/5og').<smiles>O=C(c1cc2ccccc2cc1/C=C/CCCCCCCCO)N1CCCC1</smiles><smiles>C/C(=C\CCCCCCCO)c1cc2ccccc2cc1C(=O)N1CCCC1</smiles>

Pale yellow liquid; eluent (hexane, $40 \%$ ethyl acetate). The reaction scale is $50 \mathrm{mg}, 59 \mathrm{mg}$ of product was isolated and yield is $70 \%$.

${ }^{1} \mathrm{H}$ NMR $\left(400 \mathrm{MHz}, \mathrm{CDCl}_{3}\right) \delta 7.90(\mathrm{~s}, 1 \mathrm{H}), 7.82-7.75(\mathrm{~m}, 5 \mathrm{H}), 7.70(\mathrm{~d}, \mathrm{~J}=12.4 \mathrm{~Hz}, 2 \mathrm{H})$, $7.49-7.40(\mathrm{~m}, 4 \mathrm{H}), 6.51(\mathrm{~d}, \mathrm{~J}=15.6 \mathrm{~Hz}, 1 \mathrm{H}), 6.38-6.27(\mathrm{~m}, 1 \mathrm{H}), 5.64(\mathrm{t}, \mathrm{J}=7.0 \mathrm{~Hz}, 0.6 \mathrm{H})$, $3.70(\mathrm{t}, \mathrm{J}=6.9 \mathrm{~Hz}, 2.7 \mathrm{H}), 3.64-3.59(\mathrm{~m}, 5 \mathrm{H}), 3.10(\mathrm{t}, \mathrm{J}=6.1 \mathrm{~Hz}, 3 \mathrm{H}), 2.26-2.20(\mathrm{~m}, 2.6 \mathrm{H})$, $2.19-2.14(\mathrm{~m}, 2.4 \mathrm{H}), 2.07(\mathrm{~s}, 2.2 \mathrm{H}), 1.93(\mathrm{dt}, \mathrm{J}=13.7,6.8 \mathrm{~Hz}, 4.6 \mathrm{H}), 1.80(\mathrm{dt}, \mathrm{J}=12.7,6.4$ $\mathrm{Hz}, 4.4 \mathrm{H}), 1.57-1.52(\mathrm{~m}, 3 \mathrm{H}), 1.46(\mathrm{~d}, \mathrm{~J}=6.9 \mathrm{~Hz}, 3 \mathrm{H}), 1.34-1.29(\mathrm{~m}, 10.9 \mathrm{H}), 1.25(\mathrm{~s}, 4.4 \mathrm{H})$.

${ }^{13} \mathrm{C} \mathrm{NMR}\left(101 \mathrm{MHz}, \mathrm{CDCl}_{3}\right) \delta 170.2,169.7,140.0,135.0,134.9,134.3,134.1,133.5,133.3$, 132.1, 132.0, 131.7, 131.6, 127.8, 127.7, 127.6, 126.7, 126.2, 126.0, 125.5, 124.8, 62.9, 48.3, 45.6, 36.6, 33.2, 32.7, 32.6, 29.6, 29.3, 29.3, 29.3, 29.2, 28.9, 28.7, 28.2 , 25.9, 25.6, 24.6, 24.52, 17.3, 14.1.

HRMS (ESI-TOF) m/z: [M + Na $]^{+}$Calcd for $\mathrm{C}_{25} \mathrm{H}_{33} \mathrm{NO}_{2} \mathrm{Na} 402.2409$; Found 402.2411.

(E)-(2-(10-Hydroxydec-1-en-1-yl)-3,6-dimethoxyphenyl)(pyrrolidin-1-yl)methanone (5wg).

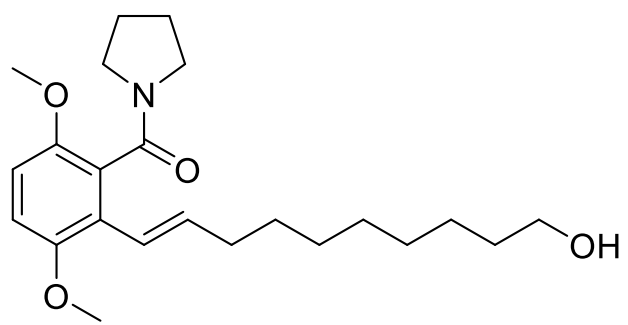

Pale yellow liquid; eluent (hexane, $40 \%$ ethyl acetate). The reaction scale is $50 \mathrm{mg}, 25 \mathrm{mg}$ of product was isolated and yield is $30 \%$.

${ }^{1} \mathrm{H}$ NMR $\left(400 \mathrm{MHz}, \mathrm{CDCl}_{3}\right) \delta 6.82(\mathrm{~d}, J=9.0 \mathrm{~Hz}, 1 \mathrm{H}), 6.74(\mathrm{~d}, J=9.0 \mathrm{~Hz}, 1 \mathrm{H}), 6.33(\mathrm{~d}, J=$ $16.0 \mathrm{~Hz}, 1 \mathrm{H}), 6.23-6.11(\mathrm{~m}, 1 \mathrm{H}), 3.79(\mathrm{~s}, 3 \mathrm{H}), 3.76(\mathrm{~s}, 3 \mathrm{H}), 3.61(\mathrm{~d}, J=6.0 \mathrm{~Hz}, 2 \mathrm{H}), 3.08(\mathrm{t}$, $J=5.9 \mathrm{~Hz}, 2 \mathrm{H}), 2.13(\mathrm{~d}, J=6.8 \mathrm{~Hz}, 2 \mathrm{H}), 1.91(\mathrm{tdd}, J=21.4,20.6,9.9 \mathrm{~Hz}, 8 \mathrm{H}), 1.54(\mathrm{~d}, J=$ $6.4 \mathrm{~Hz}, 2 \mathrm{H}), 1.30(\mathrm{~s}, 10 \mathrm{H})$.

${ }^{13} \mathrm{C} \mathrm{NMR}\left(101 \mathrm{MHz}, \mathrm{CDCl}_{3}\right) \delta 167.7,151.5,149.5,137.1,124.7,122.2,113.6,112.1,110.0$, 63.0, 56.3, 56.2, 47.6, 45.7, 34.0, 32.6, 29.3, 29.3, 29.2, 28.9, 25.6, 25.6, 24.6.

HRMS (ESI-TOF) m/z: [M + H] $]^{+}$Calcd for $\mathrm{C}_{23} \mathrm{H}_{35} \mathrm{NO}_{4} \mathrm{H}$ 390.2644; Found 390.2648. 
<smiles>Cc1ccc(C(=O)N2CCCC2)c(/C=C/CCOc2cc(=O)oc3ccccc23)c1</smiles>

Pale yellow solid; eluent (hexane, $40 \%$ ethyl acetate). The reaction scale is $50 \mathrm{mg}, 70 \mathrm{mg}$ of product was isolated and yield is $65 \%$.

${ }^{1} \mathrm{H}$ NMR (400 MHz, DMSO) $\delta 7.77(\mathrm{~d}, J=7.6 \mathrm{~Hz}, 1 \mathrm{H}), 7.63(\mathrm{t}, J=7.6 \mathrm{~Hz}, 1 \mathrm{H}), 7.41(\mathrm{~s}, 1 \mathrm{H})$, $7.37(\mathrm{~d}, J=8.3 \mathrm{~Hz}, 1 \mathrm{H}), 7.32(\mathrm{t}, J=7.3 \mathrm{~Hz}, 1 \mathrm{H}), 7.07(\mathrm{~s}, 2 \mathrm{H}), 6.49(\mathrm{~d}, J=15.9 \mathrm{~Hz}, 1 \mathrm{H}), 6.42$ $-6.30(\mathrm{~m}, 1 \mathrm{H}), 5.91(\mathrm{~s}, 1 \mathrm{H}), 4.30(\mathrm{t}, J=5.3 \mathrm{~Hz}, 2 \mathrm{H}), 3.42-3.38(\mathrm{~m}, 2 \mathrm{H}), 2.94(\mathrm{t}, J=6.3 \mathrm{~Hz}$, $2 \mathrm{H}), 2.72(\mathrm{~d}, J=5.7 \mathrm{~Hz}, 2 \mathrm{H}), 2.29(\mathrm{~s}, 3 \mathrm{H}), 1.76-1.71(\mathrm{~m}, 2 \mathrm{H}), 1.67-1.61(\mathrm{~m}, 2 \mathrm{H})$.

${ }^{13} \mathrm{C}$ NMR (101 MHz, DMSO) $\delta 168.2,164.9,161.7,152.8,138.1,134.0,132.8,128.8,128.2$, $128.0,126.2$, 125.9, 124.2, 122.8, 116.4, 115.2, 90.6, 68.6, 47.7, 45.1, 31.9, 25.5, 24.0, 20.9.

HRMS (ESI-TOF) m/z: $[\mathrm{M}+\mathrm{Na}]^{+}$Calcd for $\mathrm{C}_{25} \mathrm{H}_{25} \mathrm{NO}_{4} \mathrm{Na} 426.1681$; Found 426.1683

(E)-4-((4-(3-(Pyrrolidine-1-carbonyl)naphthalen-2-yl)but-3-en-1-yl)oxy)-2H-chromen-2one (5oh).<smiles>O=C(c1cc2ccccc2cc1/C=C/CCOc1cc(=O)oc2ccccc12)N1CCCC1</smiles>

Pale yellow solid; eluent (hexane, $40 \%$ ethyl acetate). The reaction scale is $50 \mathrm{mg}, 76 \mathrm{mg}$ of product was isolated and yield is $78 \%$.

${ }^{1} \mathrm{H}$ NMR (400 MHz, DMSO) $\delta 8.11(\mathrm{~s}, 1 \mathrm{H}), 7.89(\mathrm{t}, J=6.6 \mathrm{~Hz}, 2 \mathrm{H}), 7.79(\mathrm{~d}, J=10.4 \mathrm{~Hz}, 2 \mathrm{H})$, $7.62(\mathrm{t}, J=7.3 \mathrm{~Hz}, 1 \mathrm{H}), 7.53-7.46(\mathrm{~m}, 2 \mathrm{H}), 7.36(\mathrm{~d}, J=8.1 \mathrm{~Hz}, 1 \mathrm{H}), 7.32(\mathrm{t}, J=7.4 \mathrm{~Hz}, 1 \mathrm{H})$, $6.66(\mathrm{~d}, J=15.8 \mathrm{~Hz}, 1 \mathrm{H}), 6.50(\mathrm{dd}, J=14.5,7.3 \mathrm{~Hz}, 1 \mathrm{H}), 5.93(\mathrm{~s}, 1 \mathrm{H}), 4.34(\mathrm{~d}, J=6.1 \mathrm{~Hz}$, 2H), $3.45(\mathrm{~s}, 2 \mathrm{H}), 2.97(\mathrm{t}, J=6.8 \mathrm{~Hz}, 2 \mathrm{H}), 2.78(\mathrm{q}, J=6.4 \mathrm{~Hz}, 2 \mathrm{H}), 1.78-1.70(\mathrm{~m}, 2 \mathrm{H}), 1.67$ $-1.60(\mathrm{~m}, 2 \mathrm{H})$.

${ }^{13} \mathrm{C}$ NMR (101 MHz, DMSO) $\delta$ 168.0, 164.9, 161.7, 152.8, 135.1, 132.8, 132.8, 131.7, 131.1, $129.0,128.9,127.7,126.9,126.4,125.3,124.8,124.2,122.9,116.4,115.2$, 90.6, 68.7, 47.8, $45.2,32.0,25.5,24.0$.

HRMS (ESI-TOF) m/z: [M + Na $]^{+}$Calcd for $\mathrm{C}_{28} \mathrm{H}_{25} \mathrm{NO}_{4} \mathrm{Na} 462.1681$; Found 462.1681 . 
(E)-2-(4-(3-(Pyrrolidine-1-carbonyl)naphthalen-2-yl)but-3-en-1-yl)benzo[d]isothiazol3(2H)-one 1,1-dioxide (5oi).<smiles>O=C(c1cc2ccccc2cc1/C=C/CCN1C(=O)c2ccccc2S1(=O)=O)N1CCCC1</smiles>

Pale yellow semi-solid; eluent (hexane, 35\% ethyl acetate). The reaction scale is $50 \mathrm{mg}, 69 \mathrm{mg}$ of product was isolated and yield is $68 \%$.

${ }^{1} \mathrm{H}$ NMR $\left(400 \mathrm{MHz}, \mathrm{CDCl}_{3}\right) \delta 8.05-8.01(\mathrm{~m}, 1 \mathrm{H}), 7.96(\mathrm{~s}, 1 \mathrm{H}), 7.92-7.89(\mathrm{~m}, 1 \mathrm{H}), 7.85(\mathrm{~d}$, $\mathrm{J}=7.3 \mathrm{~Hz}, 1 \mathrm{H}), 7.81(\mathrm{~d}, \mathrm{~J}=8.4 \mathrm{~Hz}, 2 \mathrm{H}), 7.76(\mathrm{~d}, \mathrm{~J}=7.3 \mathrm{~Hz}, 1 \mathrm{H}), 7.70(\mathrm{~s}, 1 \mathrm{H}), 7.46(\mathrm{q}, \mathrm{J}=$ 8.0, $7.4 \mathrm{~Hz}, 2 \mathrm{H}), 6.67(\mathrm{~d}, \mathrm{~J}=15.6 \mathrm{~Hz}, 1 \mathrm{H}), 6.42-6.26(\mathrm{~m}, 1 \mathrm{H}), 3.92(\mathrm{t}, \mathrm{J}=7.6 \mathrm{~Hz}, 2 \mathrm{H}), 3.67$ $(\mathrm{t}, \mathrm{J}=7.6 \mathrm{~Hz}, 2 \mathrm{H}), 3.06(\mathrm{t}, \mathrm{J}=7.1 \mathrm{~Hz}, 2 \mathrm{H}), 2.79(\mathrm{~d}, \mathrm{~J}=6.4 \mathrm{~Hz}, 2 \mathrm{H}), 1.93(\mathrm{t}, \mathrm{J}=7.2 \mathrm{~Hz}, 2 \mathrm{H})$, $1.74(\mathrm{t}, \mathrm{J}=7.3 \mathrm{~Hz}, 2 \mathrm{H})$.

${ }^{13} \mathrm{C} \mathrm{NMR}\left(101 \mathrm{MHz}, \mathrm{CDCl}_{3}\right) \delta 169.3,158.8,137.6,135.0,134.7,134.3,133.3,132.2,131.4$, 130.0, 128.4, 127.9, 127.7, 127.2, 126.8, 126.3, 125.4, 125.2, 125.1, 120.8, 48.3, 45.5, 38.8, $32.3,25.8,24.5$.

HRMS (ESI-TOF) m/z: [M + Na $]^{+}$Calcd for $\mathrm{C}_{26} \mathrm{H}_{24} \mathrm{~N}_{2} \mathrm{SO}_{4} \mathrm{Na} 483.1354$; Found 483.1354 .

(E)-2-(4-(3-(Pyrrolidine-1-carbonyl)naphthalen-2-yl)but-3-en-1-yl)isoindoline-1,3dione/(E)-2-(3-(3-(Pyrrolidine-1-carbonyl)naphthalen-2-yl)but-2-en-1-yl)isoindoline-1,3dione $(\mathbf{5 o j} / \mathbf{5 o j}$ ').<smiles>C/C(=C\CN1C(=O)c2ccccc2C1=O)c1cc2ccccc2cc1C(=O)N1CCCC1</smiles>

Pale yellow semi-solid; eluent (hexane, 35\% ethyl acetate). The reaction scale is $50 \mathrm{mg}, 72 \mathrm{mg}$ of product was isolated and yield is $77 \%$.

${ }^{1} \mathrm{H}$ NMR $\left(400 \mathrm{MHz}, \mathrm{CDCl}_{3}\right) \delta 7.91(\mathrm{~s}, 1.1 \mathrm{H}), 7.82(\mathrm{~d}, J=5.6 \mathrm{~Hz}, 4.9 \mathrm{H}), 7.76(\mathrm{~d}, J=7.9 \mathrm{~Hz}$, 2.1H), $7.68(\mathrm{~s}, 4.2 \mathrm{H}), 7.46(\mathrm{dd}, J=15.3,7.4 \mathrm{~Hz}, 3.3 \mathrm{H}), 6.58(\mathrm{~d}, J=15.6 \mathrm{~Hz}, 1 \mathrm{H}), 6.32(\mathrm{dd}, J$ $=15.0,7.5 \mathrm{~Hz}, 1 \mathrm{H}), 5.66(\mathrm{t}, J=6.6 \mathrm{~Hz}, 0.3 \mathrm{H}), 4.46(\mathrm{~d}, J=6.7 \mathrm{~Hz}, 0.7 \mathrm{H}), 3.86(\mathrm{t}, J=6.8 \mathrm{~Hz}$, $2.4 \mathrm{H}), 3.67(\mathrm{t}, J=6.8 \mathrm{~Hz}, 2.4 \mathrm{H}), 3.52(\mathrm{t}, J=6.9 \mathrm{~Hz}, 0.7 \mathrm{H}), 3.09(\mathrm{t}, J=6.5 \mathrm{~Hz}, 0.7 \mathrm{H}), 3.03(\mathrm{t}$, $J=6.6 \mathrm{~Hz}, 2.2 \mathrm{H}), 2.64(\mathrm{q}, J=6.6 \mathrm{~Hz}, 2.1 \mathrm{H}), 2.29(\mathrm{~s}, 1 \mathrm{H}), 1.96-1.85(\mathrm{~m}, 3.4 \mathrm{H}), 1.80-1.72$ $(\mathrm{m}, 4.5 \mathrm{H})$. 
${ }^{13} \mathrm{C}$ NMR $\left(101 \mathrm{MHz}, \mathrm{CDCl}_{3}\right) \delta 169.6,169.3,168.3,167.9,139.5,138.8,135.0,134.8,134.0$, $133.9,133.8,133.3,133.2,132.2,132.1,132.0,131.5,129.4,129.2,127.9,127.8,127.7,127.1$, $126.9,126.8,126.4,126.3,126.3,125.4,125.1,123.9,123.2,123.1,48.3,45.6,45.5,37.5$, $36.1,32.5,25.9,24.6,24.3,17.8$.

HRMS (ESI-TOF) m/z: [M + Na]+ Calcd for $\mathrm{C}_{27} \mathrm{H}_{24} \mathrm{~N}_{2} \mathrm{O}_{3} \mathrm{Na}$ 447.1685; Found 447.1687.

(E)-(3-(3-Phenylprop-1-en-1-yl)naphthalen-2-yl)(pyrrolidin-1-yl)methanone/(3-(3Phenylprop-1-en-2-yl)naphthalen-2-yl)(pyrrolidin-1-yl)methanone(5ok/5ok').<smiles>C=C(Cc1ccccc1)c1cc2ccccc2cc1C(=O)N1CCCC1</smiles>

Pale yellow semi-solid; eluent (hexane, $10 \%$ ethyl acetate). The reaction scale is $50 \mathrm{mg}, 41 \mathrm{mg}$ of product was isolated and yield is $54 \%$.

1H NMR (400 MHz, CDCl3) $\delta 7.84(\mathrm{~d}, \mathrm{~J}=7.7 \mathrm{~Hz}, 2 \mathrm{H}), 7.83-7.77(\mathrm{~m}, 3 \mathrm{H}), 7.76(\mathrm{~s}, 1.5 \mathrm{H})$, $7.70(\mathrm{~d}, \mathrm{~J}=4.3 \mathrm{~Hz}, 1.8 \mathrm{H}), 7.55-7.43(\mathrm{~m}, 4.3 \mathrm{H}), 7.39(\mathrm{~s}, 0.7 \mathrm{H}), 7.37(\mathrm{~s}, 1.2 \mathrm{H}), 7.34(\mathrm{~d}, \mathrm{~J}=7.8$ $\mathrm{Hz}, 2.4 \mathrm{H}), 7.29(\mathrm{~d}, \mathrm{~J}=7.4 \mathrm{~Hz}, 2.2 \mathrm{H}), 7.26(\mathrm{~s}, 1.7 \mathrm{H}), 7.22(\mathrm{~d}, \mathrm{~J}=9.7 \mathrm{~Hz}, 1.7 \mathrm{H}), 7.17(\mathrm{dd}, \mathrm{J}=$ 13.9, 5.4 Hz, 2H), $6.52(\mathrm{~d}, \mathrm{~J}=16.1 \mathrm{~Hz}, 1 \mathrm{H}), 6.34(\mathrm{dt}, \mathrm{J}=15.1,7.0 \mathrm{~Hz}, 1 \mathrm{H}), 5.30(\mathrm{~s}, 0.1 \mathrm{H}), 5.09$ $(\mathrm{s}, 0.1 \mathrm{H}), 3.80(\mathrm{~d}, \mathrm{~J}=7.7 \mathrm{~Hz}, 1.8 \mathrm{H}), 3.65(\mathrm{q}, \mathrm{J}=6.6 \mathrm{~Hz}, 3.3 \mathrm{H}), 3.18(\mathrm{dt}, \mathrm{J}=13.7,6.7 \mathrm{~Hz}$, $2.6 \mathrm{H}), 3.11(\mathrm{~d}, \mathrm{~J}=6.4 \mathrm{~Hz}, 0.7 \mathrm{H}), 3.05(\mathrm{q}, \mathrm{J}=6.8 \mathrm{~Hz}, 0.6 \mathrm{H}), 2.86-2.79(\mathrm{~m}, 0.6 \mathrm{H}), 2.71(\mathrm{t}, \mathrm{J}=$ $7.6 \mathrm{~Hz}, 0.5 \mathrm{H}), 2.34(\mathrm{~s}, 1.7 \mathrm{H}), 2.07-2.00(\mathrm{~m}, 0.6 \mathrm{H}), 1.91(\mathrm{dt}, \mathrm{J}=13.5,6.7 \mathrm{~Hz}, 2.5 \mathrm{H}), 1.79$ (dd, $\mathrm{J}=12.9,6.3 \mathrm{~Hz}, 6 \mathrm{H}), 1.59-1.54(\mathrm{~m}, 1.5 \mathrm{H})$.

${ }^{13} \mathrm{C}$ NMR $\left(101 \mathrm{MHz}, \mathrm{CDCl}_{3}\right) \delta 170.0,169.6,142.1,140.1,137.9,137.2,137.1,136.0,134.9$, 134.4, 133.5, 133.4, 133.3, 132.0, 131.6, 131.5, 130.3, 129.4, 129.3, 128.9, 128.7, 128.6, 128.6, $128.6,128.5,128.5,128.4,128.3,128.2,128.1,127.9,127.9,127.7,127.7,127.4,127.3,127.2$, 127.1, 126.9, 126.8, 126.7, 126.6, 126.5, 126.4, 126.0, 125.7, 125.3, 115.4, 49.1, 48.4, 45.7, $45.6,36.9,35.7,32.6,32.2,26.0,25.9,25.6,24.5,24.4,19.2$.

HRMS (ESI-TOF) m/z: [M + Na $]^{+}$Calcd for $\mathrm{C}_{24} \mathrm{H}_{23} \mathrm{NONa} 364.1677$; Found 364.1678.

(3-((1S,2S,4R)-Bicyclo[2.2.1]heptan-2-yl)naphthalen-2-yl)(pyrrolidin-1-yl)methanone (6ol).<smiles>O=C(c1cc2ccccc2cc1C1C2CCC1C2)N1CCCC1</smiles> 
White solid; eluent (hexane, $10 \%$ ethyl acetate). The reaction scale is $50 \mathrm{mg}, 64 \mathrm{mg}$ of product was isolated and yield is $90 \%$.

${ }^{1} \mathrm{H}$ NMR $\left(400 \mathrm{MHz}, \mathrm{CDCl}_{3}\right) \delta 7.78(\mathrm{dd}, J=14.2,7.9 \mathrm{~Hz}, 2 \mathrm{H}), 7.73(\mathrm{~s}, 1 \mathrm{H}), 7.65(\mathrm{~s}, 1 \mathrm{H}), 7.50$ $-7.39(\mathrm{~m}, 2 \mathrm{H}), 3.71(\mathrm{~d}, J=3.0 \mathrm{~Hz}, 2 \mathrm{H}), 3.22(\mathrm{~d}, J=7.0 \mathrm{~Hz}, 1 \mathrm{H}), 3.19-3.12(\mathrm{~m}, 1 \mathrm{H}), 2.38(\mathrm{~s}$, $1 \mathrm{H}), 2.03-1.95(\mathrm{~m}, 2 \mathrm{H}), 1.85(\mathrm{~d}, J=3.7 \mathrm{~Hz}, 2 \mathrm{H}), 1.79(\mathrm{~d}, J=12.2 \mathrm{~Hz}, 1 \mathrm{H}), 1.75-1.65(\mathrm{~m}$, $3 \mathrm{H}), 1.61(\mathrm{~d}, J=10.1 \mathrm{~Hz}, 2 \mathrm{H}), 1.56(\mathrm{~d}, J=12.6 \mathrm{~Hz}, 1 \mathrm{H}), 1.42-1.33(\mathrm{~m}, 1 \mathrm{H}), 1.28(\mathrm{~d}, J=9.0$ $\mathrm{Hz}, 2 \mathrm{H})$.

${ }^{13} \mathrm{C}$ NMR $\left(101 \mathrm{MHz}, \mathrm{CDCl}_{3}\right) \delta 170.0,141.5,136.5,133.4,131.1,127.6,127.5,126.5,125.7$, $125.2,124.3,48.9,45.4,43.5,39.4,37.1,36.4,30.6,28.6,26.0,24.6$.

HRMS (ESI-TOF) m/z: [M + Na $]^{+}$Calcd for $\mathrm{C}_{22} \mathrm{H}_{25} \mathrm{NONa} 342.1834$; Found 342.1834.

(2-((1R,2R,4S)-Bicyclo[2.2.1]heptan-2-yl)-6-((1S,2S,4R)-bicyclo[2.2.1]heptan-2-yl)-4methoxyphenyl)(pyrrolidin-1-yl)methanone (6al)

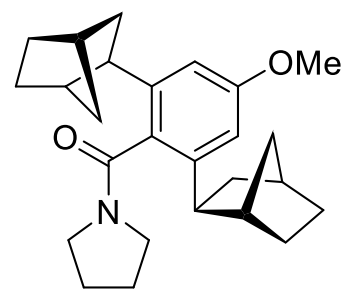

White solid; eluent (hexane, $13 \%$ ethyl acetate). The reaction scale is $50 \mathrm{mg}, 88 \mathrm{mg}$ of product was isolated and yield is $95 \%$.

${ }^{1} \mathrm{H}$ NMR $\left.\left(500 \mathrm{MHz}, \mathrm{CDCl}_{3}\right)\right)$ : exo/endo ratio $(2: 1) \delta 6.94(\mathrm{~d}, J=8.4 \mathrm{~Hz}, 2.5 \mathrm{H}), 3.72-3.60$ $(\mathrm{m}, 2.8 \mathrm{H}), 3.11(\mathrm{t}, J=6.8 \mathrm{~Hz}, 0.6 \mathrm{H}), 3.09-3.02(\mathrm{~m}, 1.3 \mathrm{H}), 3.00(\mathrm{t}, J=6.7 \mathrm{~Hz}, 0.8 \mathrm{H}), 2.70-$ $2.61(\mathrm{~m}, 1.6 \mathrm{H}), 2.51(\mathrm{ddd}, J=16.1,8.8,6.1 \mathrm{~Hz}, 1.4 \mathrm{H}), 2.32(\mathrm{~s}, 1.5 \mathrm{H}), 2.31(\mathrm{~s}, 3 \mathrm{H}), 2.02(\mathrm{t}, J$ $=3.1 \mathrm{~Hz}, 1.2 \mathrm{H}), 1.99-1.92(\mathrm{~m}, 3 \mathrm{H}), 1.91(\mathrm{~d}, J=2.9 \mathrm{~Hz}, 1.9 \mathrm{H}), 1.86-1.78(\mathrm{~m}, 4 \mathrm{H}), 1.74-$ $1.70(\mathrm{~m}, 2 \mathrm{H}), 1.61-1.57(\mathrm{~m}, 2.6 \mathrm{H}), 1.53(\mathrm{dq}, J=8.2,2.5 \mathrm{~Hz}, 6.3 \mathrm{H}), 1.31-1.26(\mathrm{~m}, 2 \mathrm{H}), 1.26$ $-1.22(\mathrm{~m}, 2.4 \mathrm{H}), 1.20-1.17(\mathrm{~m}, 2.5 \mathrm{H})$.

${ }^{13} \mathrm{C}$ NMR $\left(126 \mathrm{MHz}, \mathrm{CDCl}_{3}\right) \delta 170.2,170.1,159.6,159.4,145.1,144.9,144.8,144.6,130.6$, 130.3, 108.8, 108.8, 108.7, 108.4, 55.1, 48.0, 47.9, 45.1, 44.3, 44.2, 43.9, 43.7, 43.5, 43.2, 43.0, $42.8,41.8,41.4,39.6,39.3,37.4,37.2$, 36.7, 36.7, 36.7, 36.4, 36.3, 36.3, 31.6, 31.5, 30.95, $30.8,28.5,28.4,28.1,28.0,25.9,25.8,25.7,24.7,24.7,24.7$.

HRMS (ESI-TOF) m/z: [M + Na $]^{+}$Calcd for $\mathrm{C}_{26} \mathrm{H}_{35} \mathrm{NO}_{2} \mathrm{Na} 416.2565$; Found 416.2571.

(2-((1R,2R,4S)-Bicyclo[2.2.1]heptan-2-yl)-6-((1S,2S,4R)-bicyclo[2.2.1]heptan-2-yl)-4methylphenyl)(pyrrolidin-1-yl)methanone (6bl) 


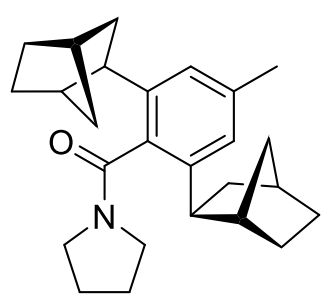

White solid; eluent (hexane, $13 \%$ ethyl acetate). The reaction scale is $50 \mathrm{mg}, 95 \mathrm{mg}$ of product was isolated and yield is $96 \%$.

${ }^{1} \mathrm{H}$ NMR $\left(500 \mathrm{MHz}, \mathrm{CDCl}_{3}\right)$ : exo/endo ratio $(2: 1) \delta 6.94(\mathrm{~d}, J=8.4 \mathrm{~Hz}, 2.5 \mathrm{H}), 3.72-3.60(\mathrm{~m}$, $2.8 \mathrm{H}), 3.11(\mathrm{t}, J=6.8 \mathrm{~Hz}, 0.6 \mathrm{H}), 3.09-3.02(\mathrm{~m}, 1.4 \mathrm{H}), 3.00(\mathrm{t}, J=6.7 \mathrm{~Hz}, 0.8 \mathrm{H}), 2.70-2.61$ $(\mathrm{m}, 1.6 \mathrm{H}), 2.51(\mathrm{ddd}, J=16.1,8.8,6.1 \mathrm{~Hz}, 1.4 \mathrm{H}), 2.32(\mathrm{~s}, 1.5 \mathrm{H}), 2.31(\mathrm{~s}, 3 \mathrm{H}), 2.02(\mathrm{t}, J=3.1$ $\mathrm{Hz}, 1.2 \mathrm{H}), 1.99-1.92(\mathrm{~m}, 3.1 \mathrm{H}), 1.91(\mathrm{~d}, J=2.9 \mathrm{~Hz}, 1.9 \mathrm{H}), 1.86-1.78(\mathrm{~m}, 4 \mathrm{H}), 1.74-1.70$ $(\mathrm{m}, 2.1 \mathrm{H}), 1.61-1.57(\mathrm{~m}, 2.6 \mathrm{H}), 1.53(\mathrm{dq}, J=8.2,2.5 \mathrm{~Hz}, 6.3 \mathrm{H}), 1.31-1.26(\mathrm{~m}, 2 \mathrm{H}), 1.26-$ $1.22(\mathrm{~m}, 2.4 \mathrm{H}), 1.20-1.17(\mathrm{~m}, 3.5 \mathrm{H})$.

${ }^{13} \mathrm{C}$ NMR $\left(101 \mathrm{MHz}, \mathrm{CDCl}_{3}\right) \delta 183.2,170.7,170.5,170.4,143.2,143.0,142.8,142.7,138.0$, 137.9, 137.6, 134.1, 134.5, 123.9, 123.8, 123.7, 48.1, 48.0, 47.9, 45.0, 44.1, 44.0, 43.7, 43.6, 43.5, 43.2, 43.1, 42.9, 41.7, 41.3, 40.4, 39.5, 39.1, 38.6, 37.3, 37.1, 36.7, 36.6, 36.6, 36.4, 36.3, 36.3, 31.6, 31.6, 30.9, 30.9, 28.5, 28.4, 28.1, 28.1, 27.8, 25.9, 25.8, 25.7, 24.7, 24.6, 24.6, 21.9.

HRMS (ESI-TOF) m/z: [M + Na $]^{+}$Calcd for $\mathrm{C}_{26} \mathrm{H}_{35} \mathrm{NONa}$ 400.2616; Found 400.2621.

(2-((1R,2R,4S)-Bicyclo[2.2.1]heptan-2-yl)-6-((1S,2S,4R)-bicyclo[2.2.1]heptan-2-yl)-4chlorophenyl)(pyrrolidin-1-yl)methanone (6dl)

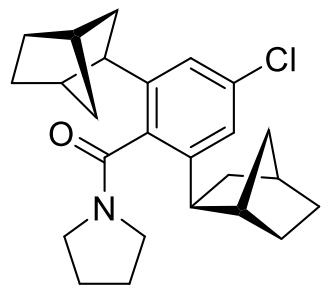

White solid; eluent (hexane, $10 \%$ ethyl acetate). The reaction scale is $50 \mathrm{mg}, 88 \mathrm{mg}$ of product was isolated and yield is $92 \%$.

${ }^{1} \mathrm{H}$ NMR $\left(500 \mathrm{MHz}, \mathrm{CDCl}_{3}\right)$ : exo/endo ratio $(2: 1) \delta 7.13-7.11(\mathrm{~m}, 1.1 \mathrm{H}), 7.10(\mathrm{~d}, J=1.9 \mathrm{~Hz}$, $1 \mathrm{H}), 3.72-3.61(\mathrm{~m}, 2.6 \mathrm{H}), 3.11-2.96(\mathrm{~m}, 2.9 \mathrm{H}), 2.66(\mathrm{ddd}, J=19.5,8.9,6.0 \mathrm{~Hz}, 1.6 \mathrm{H}), 2.50$ (ddd, $J=15.8,8.9,6.2 \mathrm{~Hz}, 1.5 \mathrm{H}), 2.02(\mathrm{p}, J=3.2 \mathrm{~Hz}, 3.5 \mathrm{H}), 1.96(\mathrm{td}, J=7.1,2.9 \mathrm{~Hz}, 2.7 \mathrm{H})$, $1.87-1.81(\mathrm{~m}, 3.9 \mathrm{H}), 1.71(\mathrm{dt}, J=7.3,2.7 \mathrm{~Hz}, 6.1 \mathrm{H}), 1.54(\mathrm{ddt}, J=6.6,4.2,2.6 \mathrm{~Hz}, 5.23 \mathrm{H})$, $1.31-1.27(\mathrm{~m}, 2.1 \mathrm{H}), 1.27-1.21(\mathrm{~m}, 3.5 \mathrm{H}), 1.19(\mathrm{ddd}, J=8.2,4.1,2.1 \mathrm{~Hz}, 2.3 \mathrm{H})$.

${ }^{13} \mathrm{C} \mathrm{NMR}\left(101 \mathrm{MHz}, \mathrm{CDCl}_{3}\right) \delta 183.3,169.4,169.2,145.6,145.5,145.3,145.1,136.0,126.4$, 126.3, 122.9, 122.1, 48.0, 48.0, 45.1, 44.2, 44.1, 43.8, 43.6, 43.5, 43.0, 42.9, 42.7, 41.8, 41.4, $40.4,39.4,39.1,38.6,37.4,37.2,36.7,36.6,36.5,36.4,36.3,36.3,36.2,31.6,31.5,30.9,30.8$, $28.4,28.3,28.0,27.9,27.8,25.9,25.8,25.7,24.6,24.6,24.5$. 
HRMS (ESI-TOF) m/z: [M + Na $]^{+}$Calcd for $\mathrm{C}_{25} \mathrm{H}_{32} \mathrm{NOClNa} 420.2070$; Found 420.2076.

(2-((1R,2R,4S)-bicyclo[2.2.1]heptan-2-yl)-6-((1S,2S,4R)-bicyclo[2.2.1]heptan-2-yl)-4bromophenyl)(pyrrolidin-1-yl)methanone (6el)<smiles></smiles>

White solid; eluent (hexane, 10\% ethyl acetate). The reaction scale is $50 \mathrm{mg}, 79 \mathrm{mg}$ of product was isolated and yield is $90 \%$.

${ }^{1} \mathrm{H}$ NMR $\left(500 \mathrm{MHz}, \mathrm{CDCl}_{3}\right)$ : exo/endo ratio $2: 1 \delta 7.31-7.29(\mathrm{~m}, 1.3 \mathrm{H}), 7.28(\mathrm{~d}, J=2.3 \mathrm{~Hz}$, $1.2 \mathrm{H}), 3.75-3.61(\mathrm{~m}, 3 \mathrm{H}), 3.11(\mathrm{t}, J=6.7 \mathrm{~Hz}, 0.8 \mathrm{H}), 3.05(\mathrm{q}, J=6.6 \mathrm{~Hz}, 1.5 \mathrm{H}), 3.01(\mathrm{t}, J=$ $6.8 \mathrm{~Hz}, 0.7 \mathrm{H}), 2.68$ (ddd, $J=19.4,8.9,6.1 \mathrm{~Hz}, 1.6 \mathrm{H}), 2.51$ (ddd, $J=15.6,8.9,6.2 \mathrm{~Hz}, 1.7 \mathrm{H}$ ), $2.05(\mathrm{p}, J=3.2 \mathrm{~Hz}, 4 \mathrm{H}), 1.99(\mathrm{td}, J=7.1,3.0 \mathrm{~Hz}, 2.6 \mathrm{H}), 1.90-1.83(\mathrm{~m}, 4.3 \mathrm{H}), 1.74(\mathrm{dd}, J=$ 7.3, 3.6 Hz, 6H), $1.57(\mathrm{dq}, J=7.6,4.0,3.4 \mathrm{~Hz}, 6.6 \mathrm{H}), 1.33-1.29(\mathrm{~m}, 2.3 \mathrm{H}), 1.29-1.23(\mathrm{~m}$, $3.6 \mathrm{H}), 1.21(\mathrm{dtd}, J=8.6,4.4,2.6 \mathrm{~Hz}, 2.8 \mathrm{H})$.

${ }^{13} \mathrm{C}$ NMR $\left(101 \mathrm{MHz}, \mathrm{CDCl}_{3}\right) \delta 183.3,169.4,169.2,145.6,145.5,145.3,145.1,135.9,126.4$, 126.3, 122.9, 122.8, 48.0, 47.9, 45.1, 44.2, 44.1, 43.8, 43.6, 43.5, 43.0, 429, 42.7, 41.8, 41.4, 40.4 , 39.4, 39.1, 38.6, 37.4, 37.3, 36.7, 36.6, 36.5, 36.4, 36.3, 36.3, 36.2, 31.6, 31.5, 30.9, 30.8, $28.4,28.3,28.0,27.9,27.8,25.9,25.8,25.7,24.6,24.6,24.5$.

HRMS (ESI-TOF) m/z: [M + Na] ${ }^{+}$Calcd for $\mathrm{C}_{25} \mathrm{H}_{32} \mathrm{NOBrNa} 464.1565$; Found 464.1564. 
${ }^{1} \mathrm{H}$ and ${ }^{13} \mathrm{C}$ NMR Spectra of Compound 3aa/3aa'. $\left(\mathrm{CDCl}_{3}\right.$ solvent was used, $\left.500 \mathrm{NMR} \mathrm{MHz}\right)$ ๆ

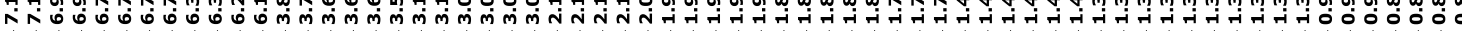
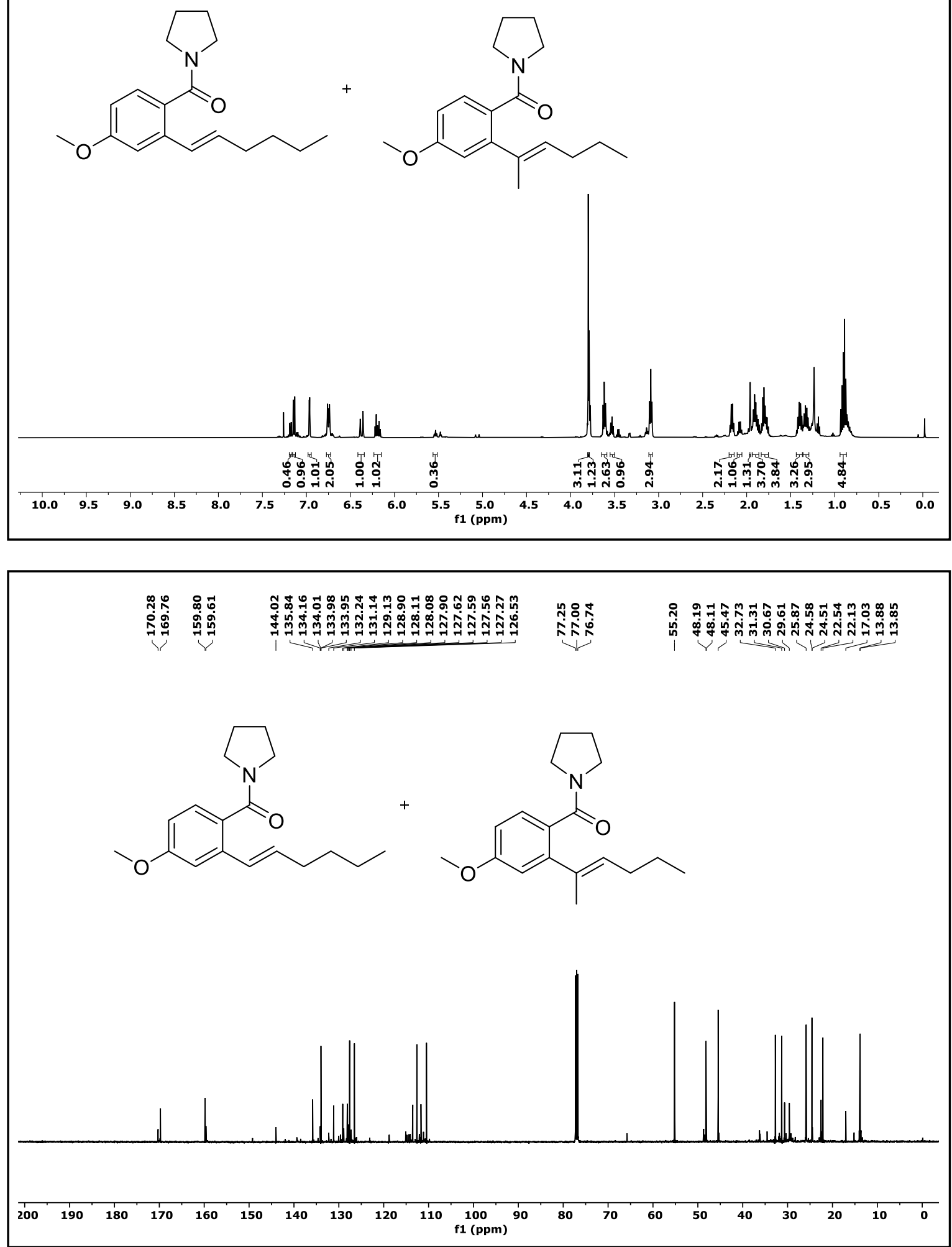
${ }^{1} \mathrm{H}$ and ${ }^{13} \mathrm{C}$ NMR Spectra of Compound 3ba/3ba'. $\left(\mathrm{CDCl}_{3}\right.$ solvent was used, $\left.500 \mathrm{NMR} \mathrm{MHz}\right)$

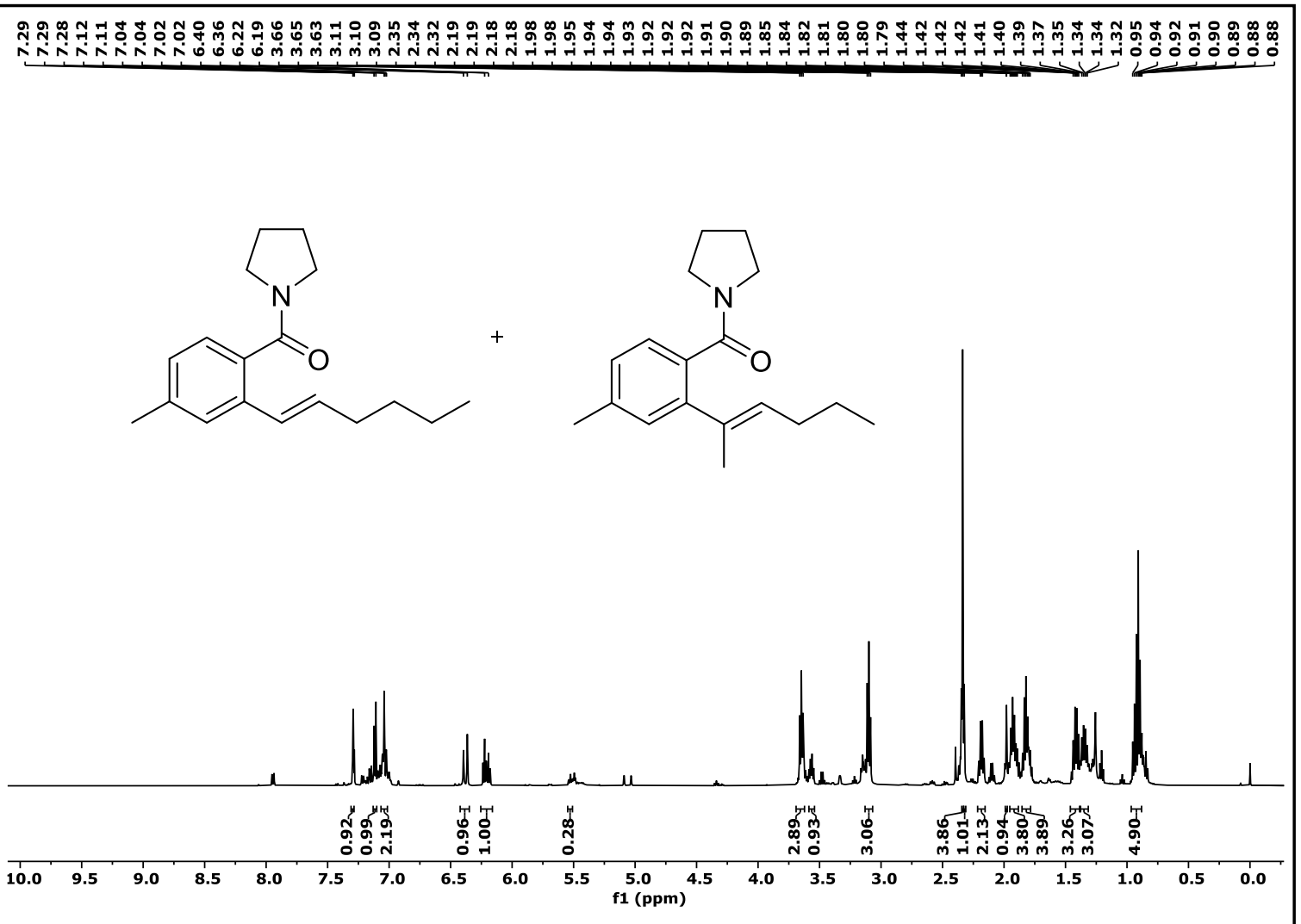

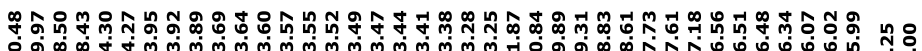

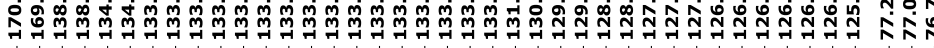

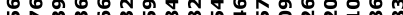

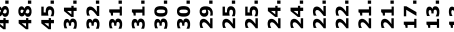<smiles>CCCC/C=C/c1cc(C)ccc1C(=O)N1CCCC1</smiles><smiles>CCC/C=C(\C)c1cc(C)ccc1C(=O)N1CCCC1</smiles>

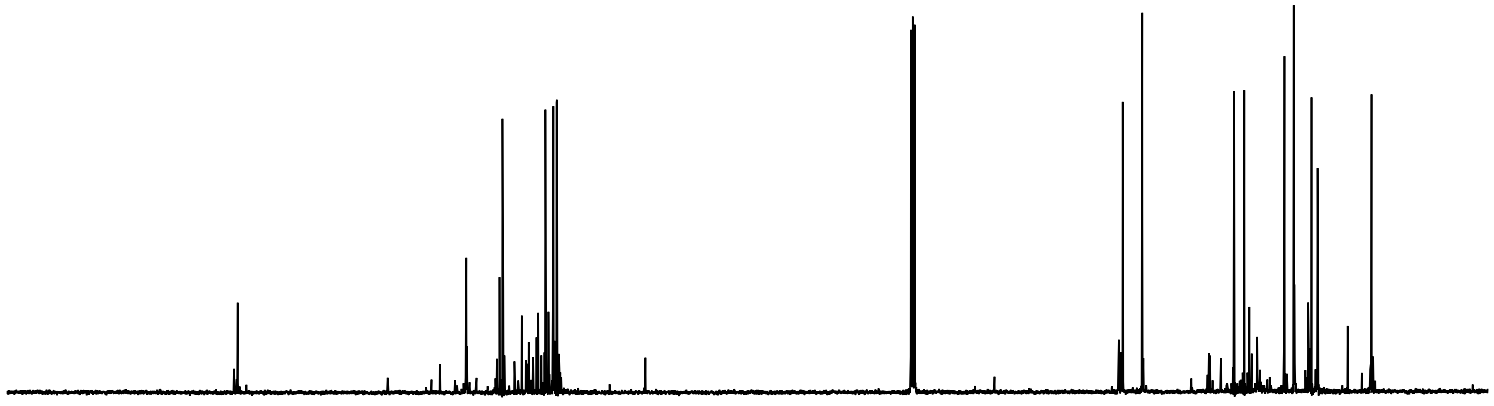

$\begin{array}{lllllllllllllllllllll}200 & 190 & 180 & 170 & 160 & 150 & 140 & 130 & 120 & 110 & \begin{array}{c}100 \\ \mathrm{f}(\mathrm{ppm})\end{array} & 90 & 80 & 70 & 60 & 50 & 40 & 30 & 20 & 10 & 0\end{array}$ 
${ }^{1} \mathrm{H}$ and ${ }^{13} \mathrm{C}$ NMR Spectra of Compound 3ca/3ca". $\left(\mathrm{CDCl}_{3}\right.$ solvent was used, $\left.400 \mathrm{NMR} \mathrm{MHz}\right)$
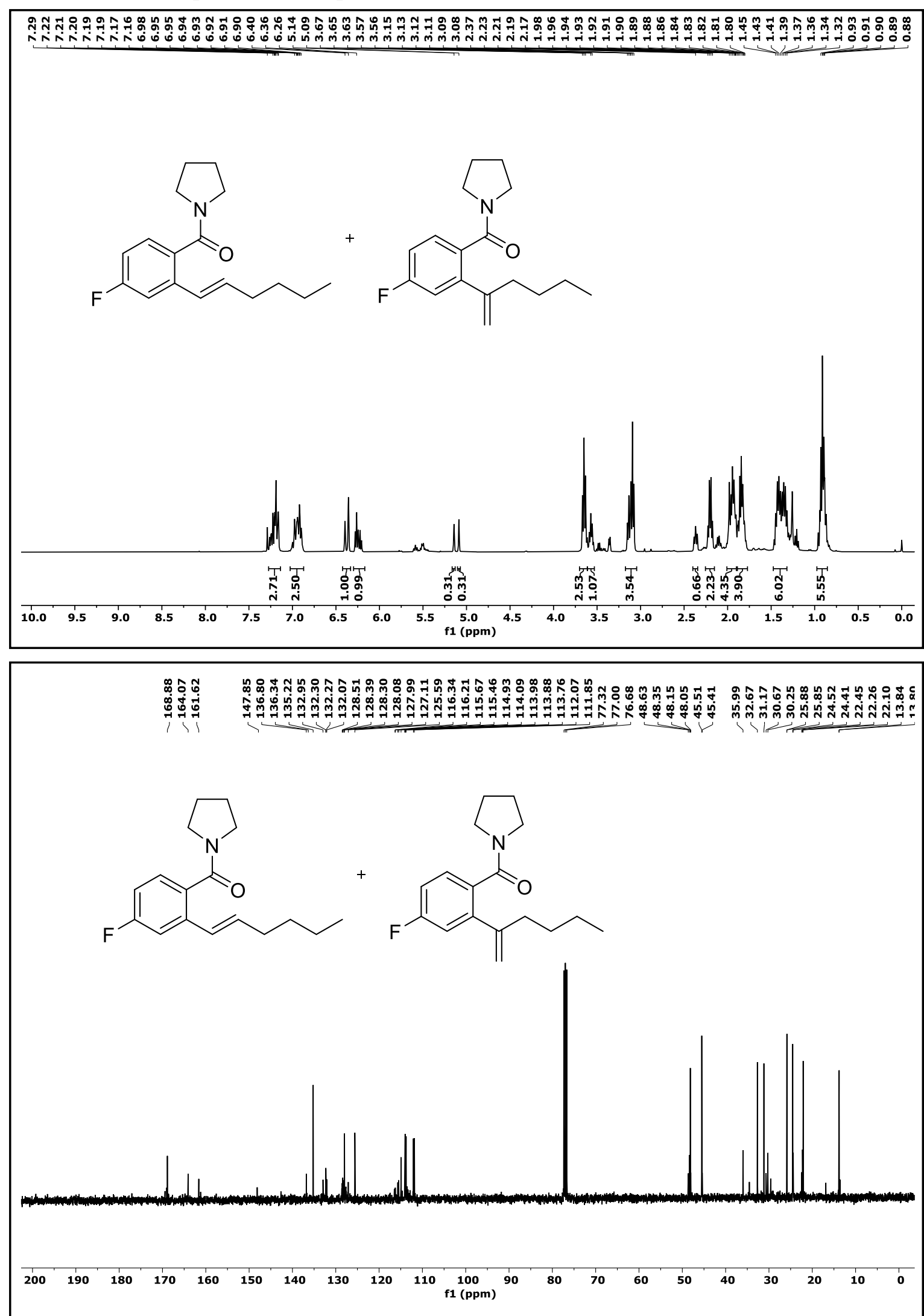
${ }^{1} \mathrm{H}$ and ${ }^{13} \mathrm{C}$ NMR Spectra of Compound 3da/3da'. $\left(\mathrm{CDCl}_{3}\right.$ solvent was used, $\left.400 \mathrm{NMR} \mathrm{MHz}\right)$

马्大<smiles>CCC/C=C/c1cc(Cl)ccc1C(=O)N1CCCC1</smiles>
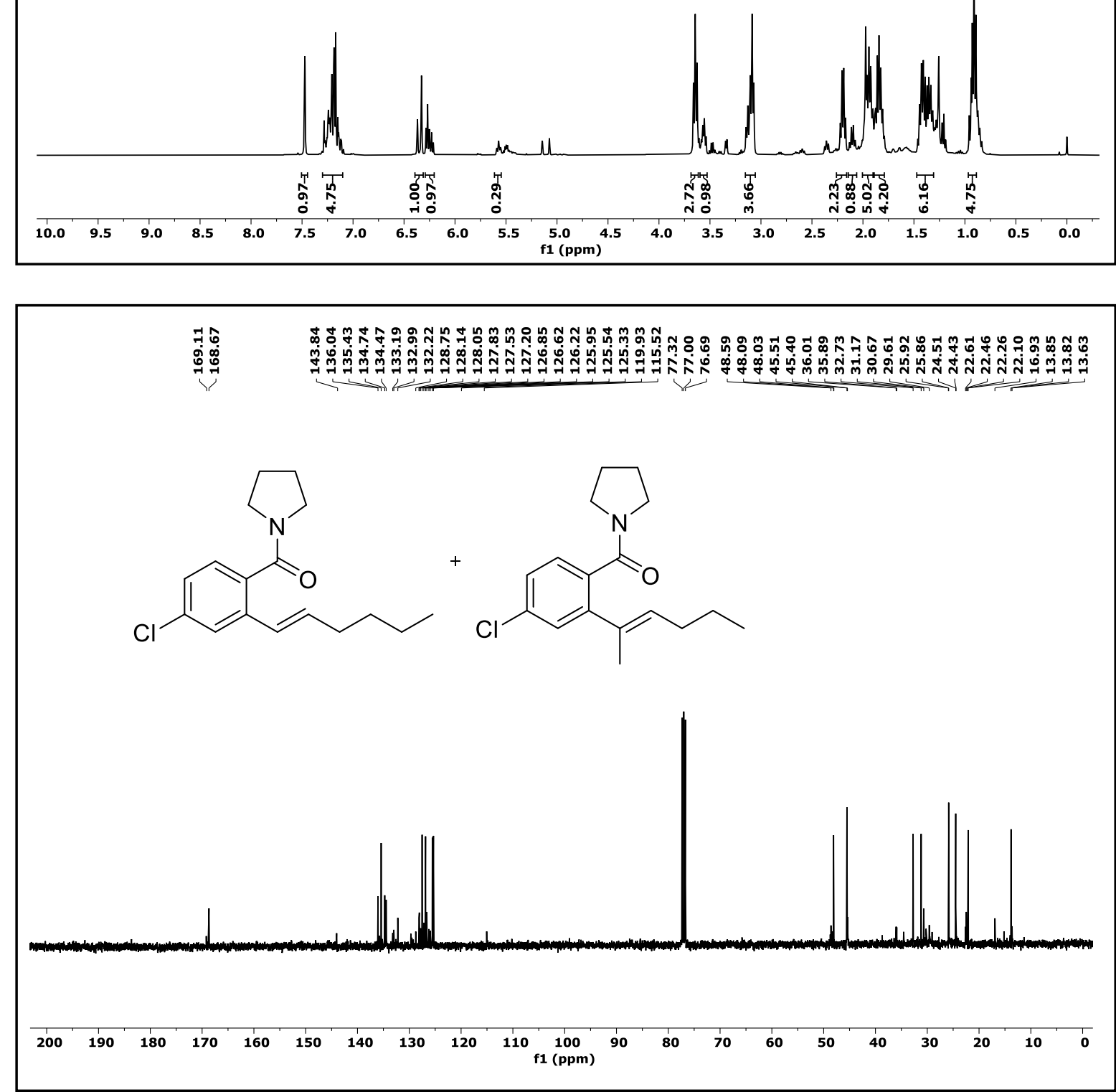
${ }^{1} \mathrm{H}$ and ${ }^{13} \mathrm{C}$ NMR Spectra of Compound 3ea/3ea'. $\left(\mathrm{CDCl}_{3}\right.$ solvent was used, $\left.400 \mathrm{NMR} \mathrm{MHz}\right)$
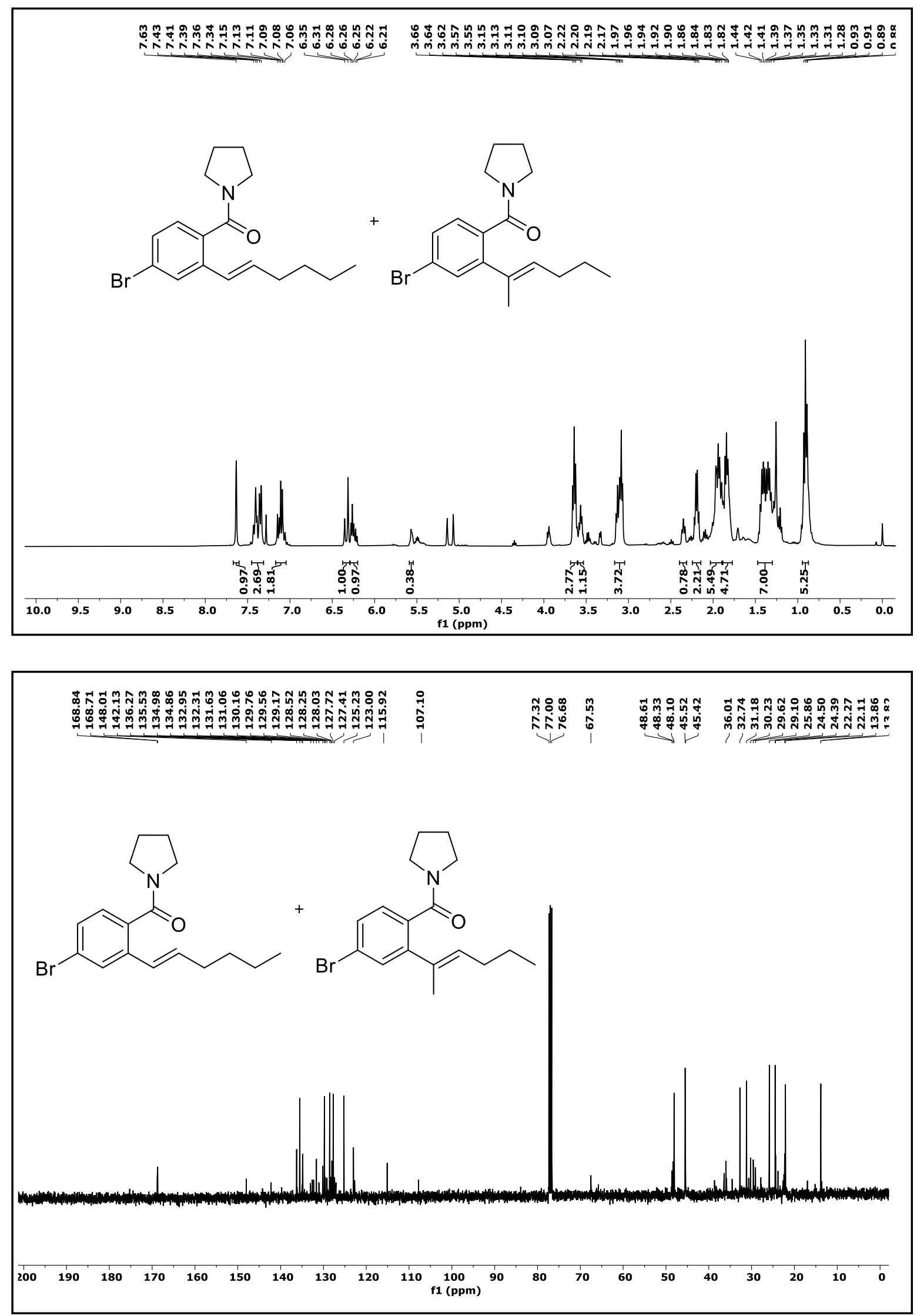
${ }^{1} \mathrm{H}$ and ${ }^{13} \mathrm{C}$ NMR Spectra of Compound 3fa/3fa". ( $\mathrm{CDCl}_{3}$ solvent was used, $\left.400 \mathrm{NMR} \mathrm{MHz}\right)$
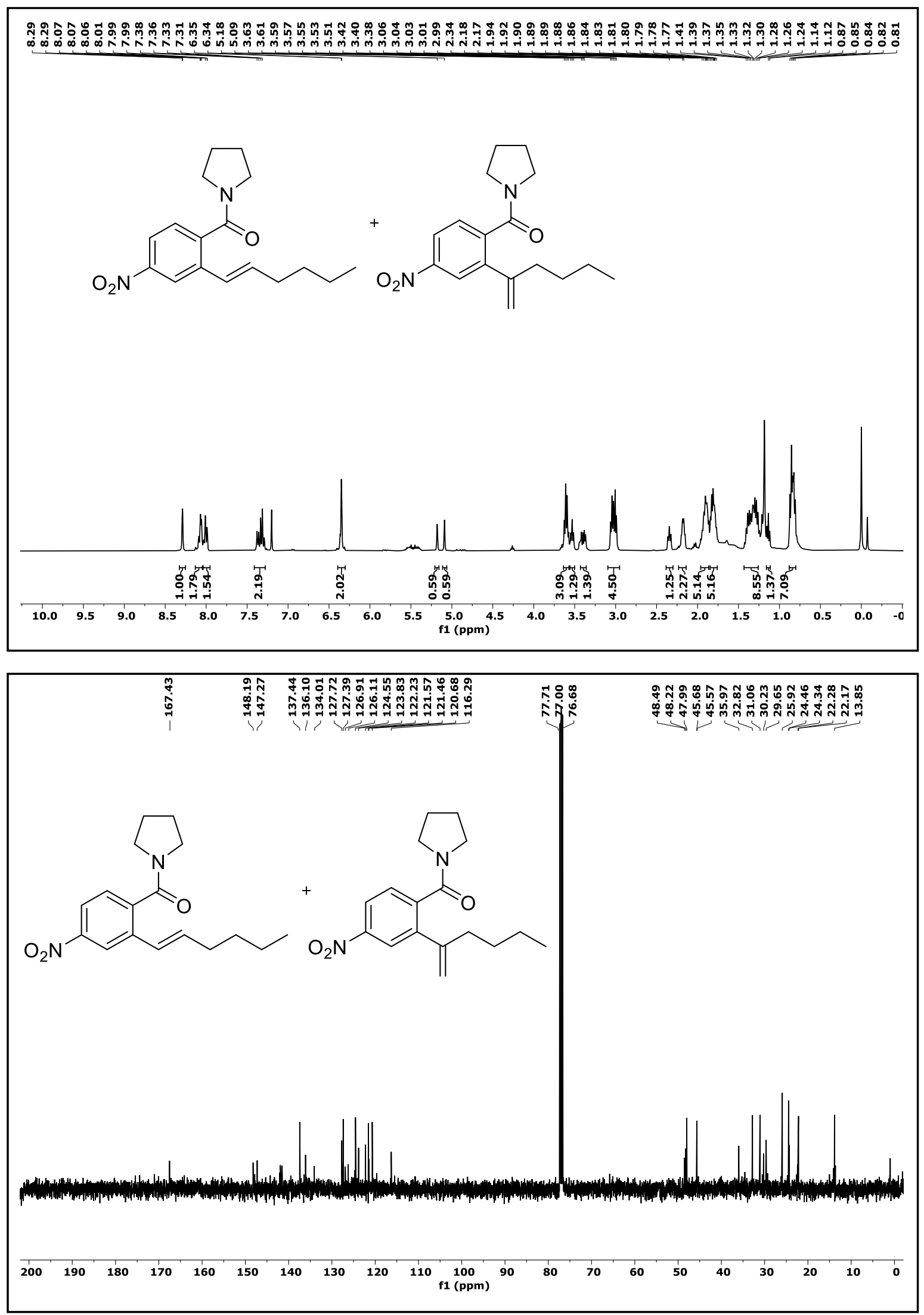
${ }^{1} \mathrm{H}$ and ${ }^{13} \mathrm{C}$ NMR Spectra of Compound 3ga/3ga'. $\left(\mathrm{CDCl}_{3}\right.$ solvent was used, $\left.400 \mathrm{NMR} \mathrm{MHz}\right)$

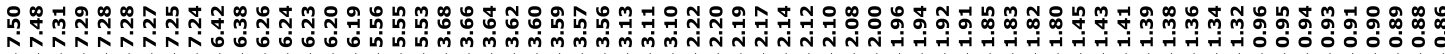<smiles>CCCC/C=C/c1ccccc1C(=O)N1CCCC1</smiles>
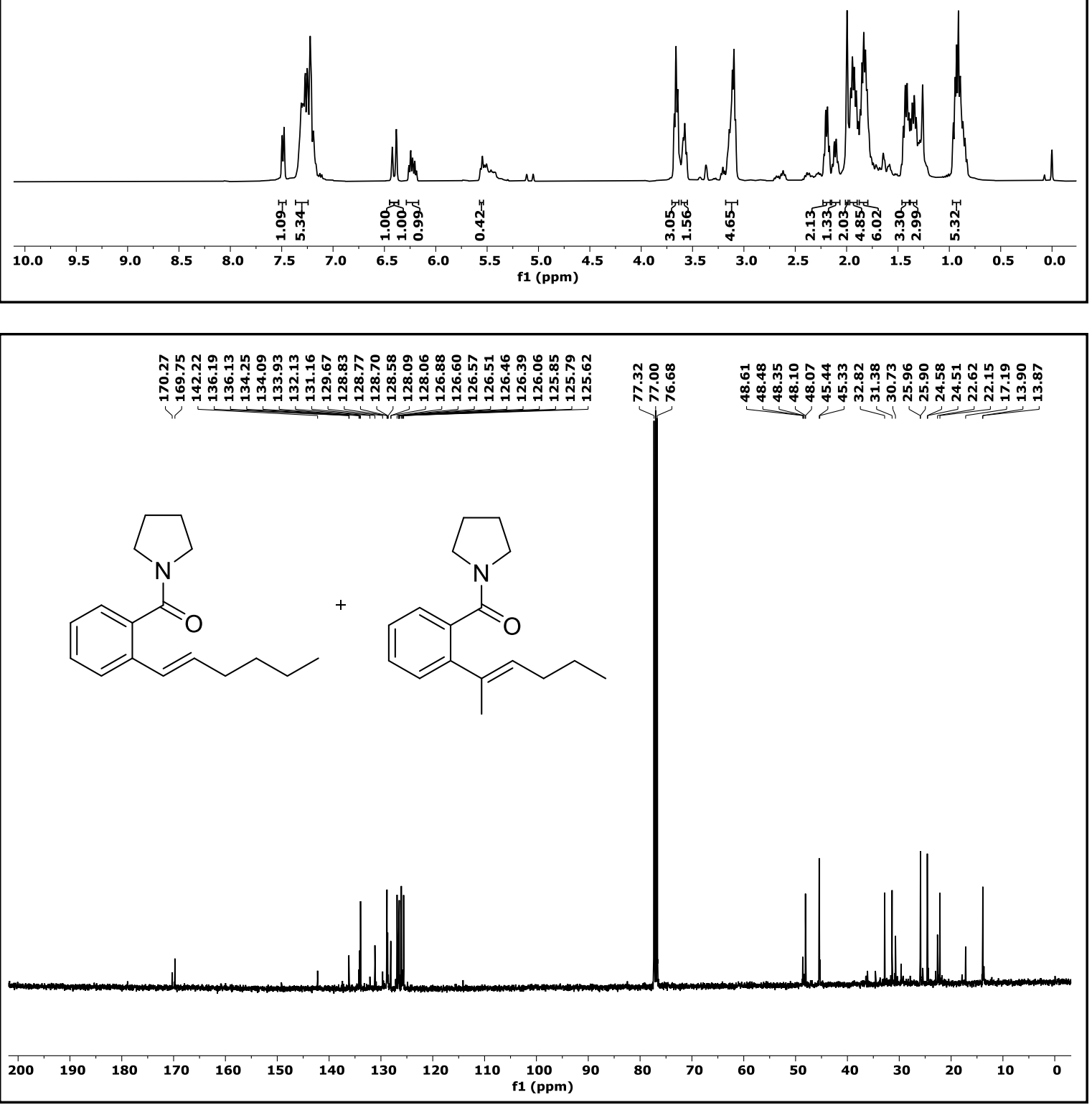
${ }^{1} \mathrm{H}$ and ${ }^{13} \mathrm{C}$ NMR Spectra of Compound 3ha/3ha'. $\left(\mathrm{CDCl}_{3}\right.$ solvent was used, $\left.400 \mathrm{NMR} \mathrm{MHz}\right)$
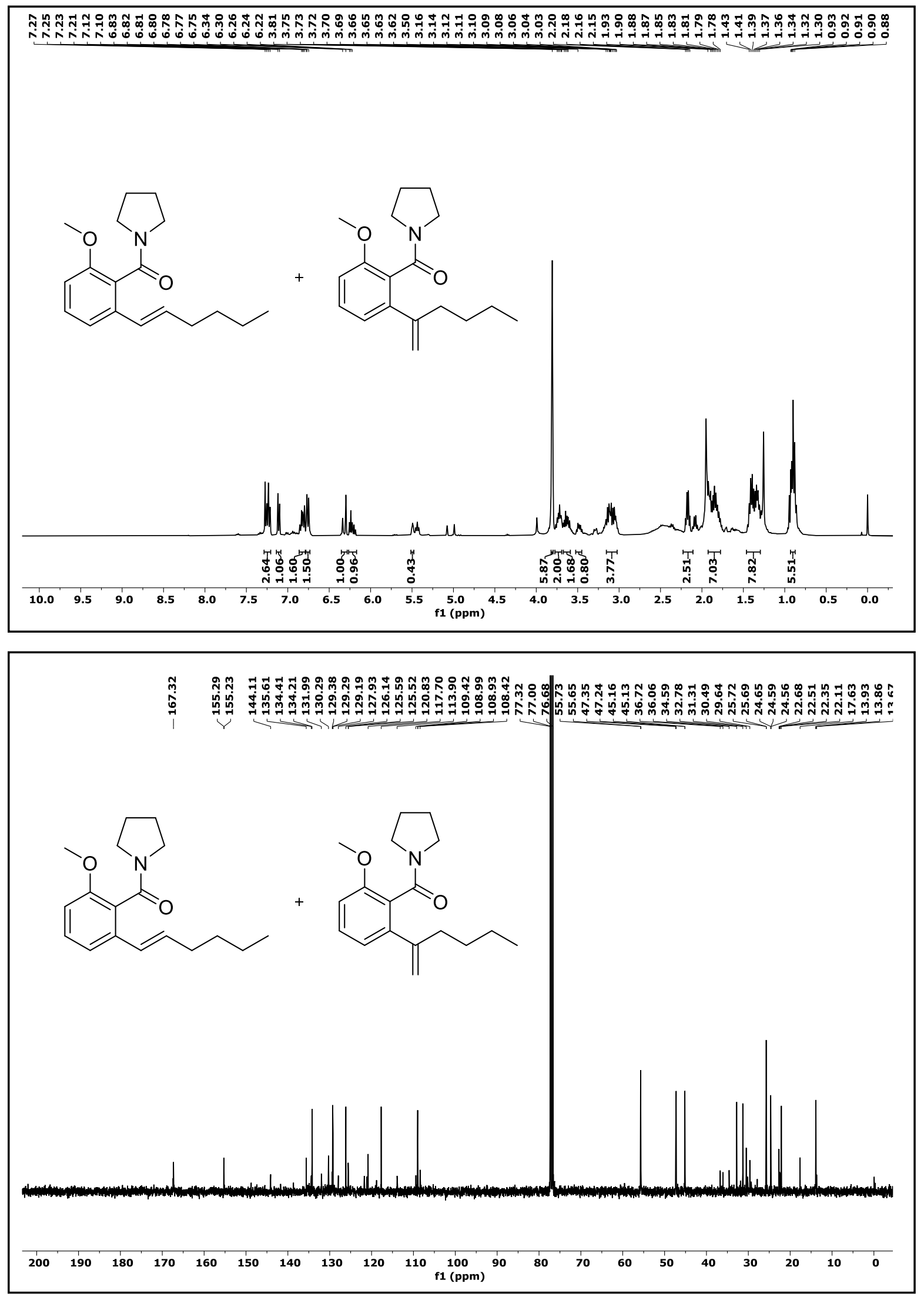
${ }^{1} \mathrm{H}$ and ${ }^{13} \mathrm{C}$ NMR Spectra of Compound 3ia/3ia". $\left(\mathrm{CDCl}_{3}\right.$ solvent was used, $\left.400 \mathrm{NMR} \mathrm{MHz}\right)$
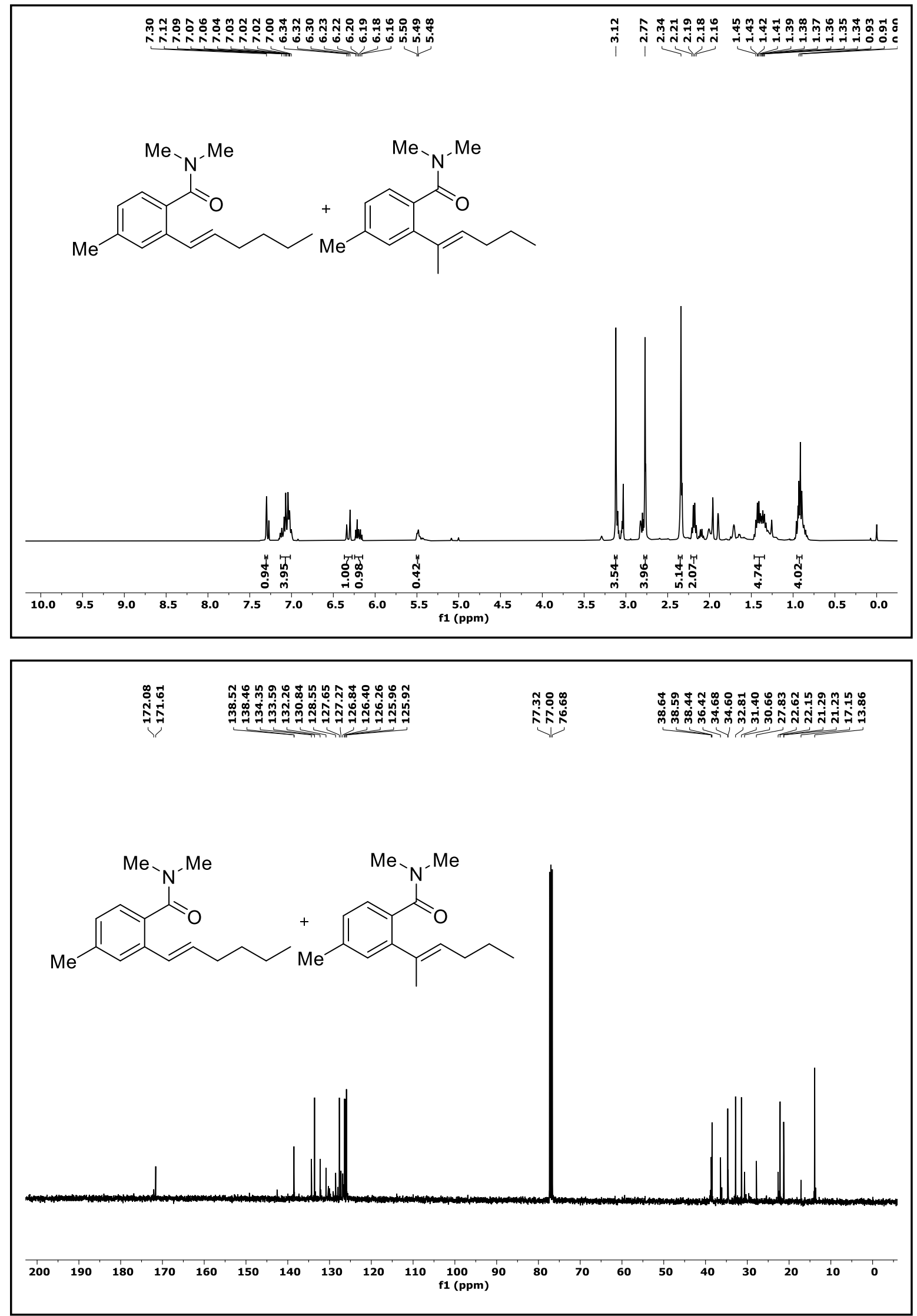
${ }^{1} \mathrm{H}$ and ${ }^{13} \mathrm{C}$ NMR Spectra of Compound 3ja/3ja'. $\left(\mathrm{CDCl}_{3}\right.$ solvent was used, $\left.400 \mathrm{NMR} \mathrm{MHz}\right)$
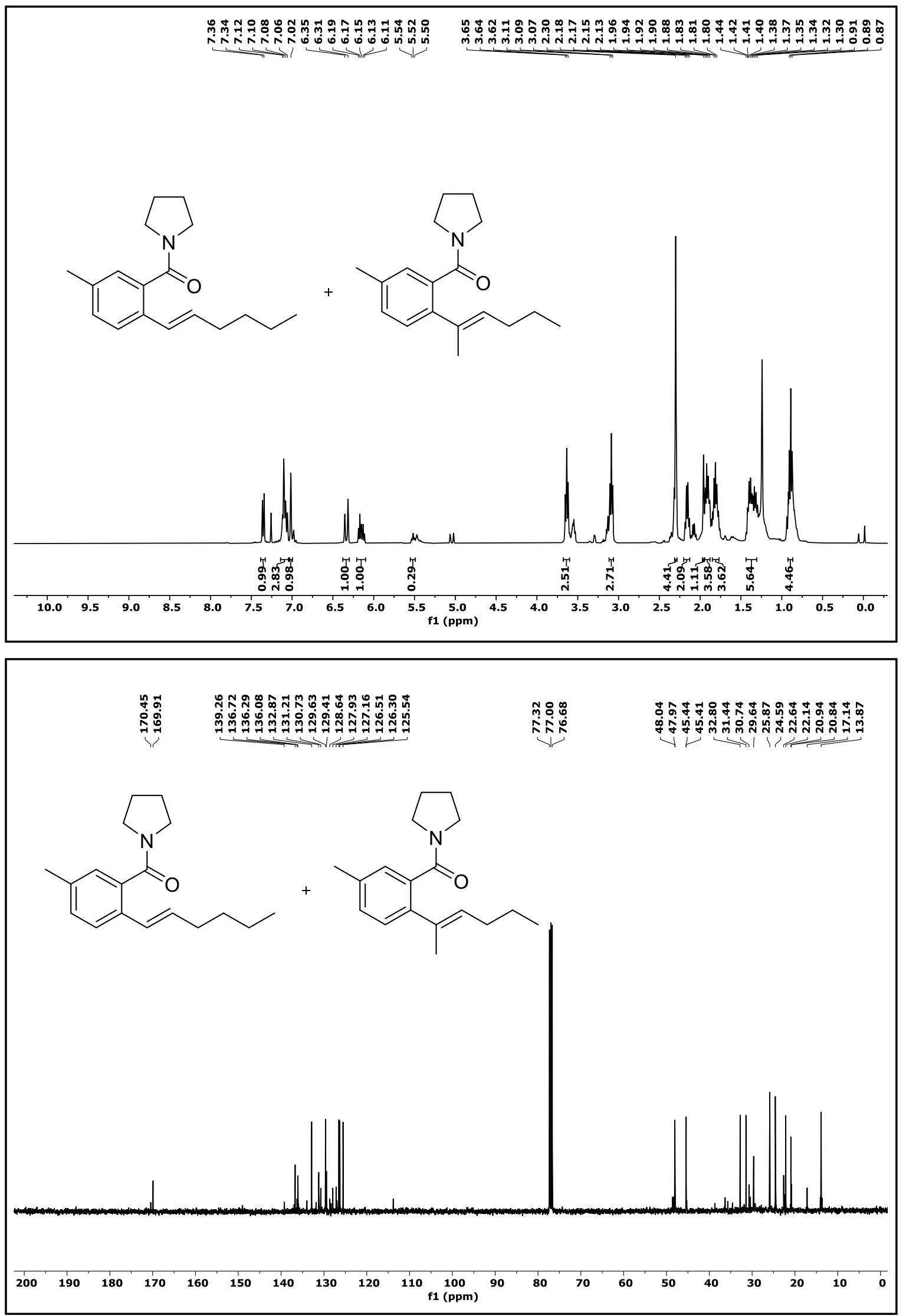
${ }^{1} \mathrm{H}$ and ${ }^{13} \mathrm{C}$ NMR Spectra of Compound 3ka/3ka”. $\left(\mathrm{CDCl}_{3}\right.$ solvent was used, $\left.400 \mathrm{NMR} \mathrm{MHz}\right)$
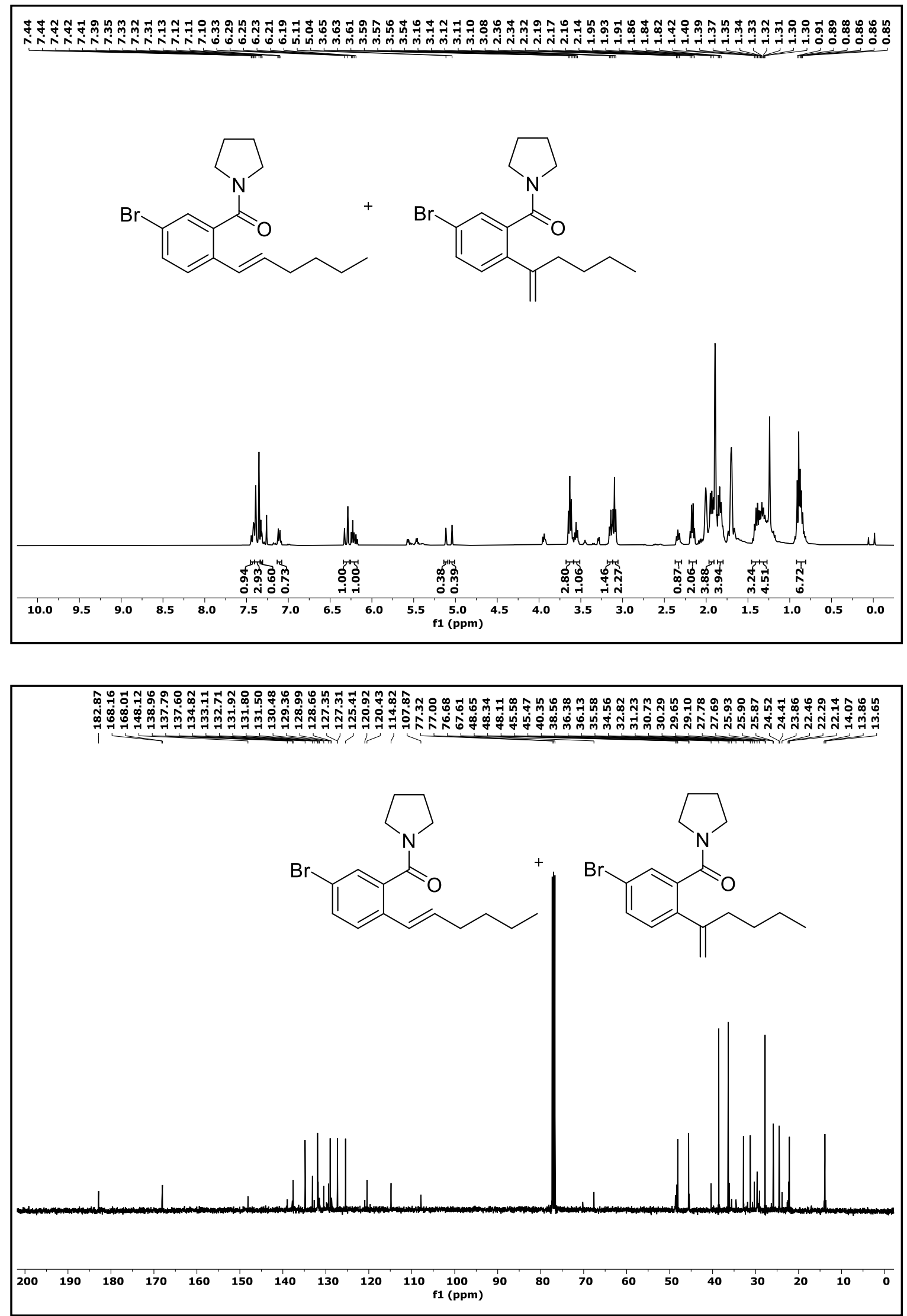
${ }^{1} \mathrm{H}$ and ${ }^{13} \mathrm{C}$ NMR Spectra of Compound 3la/3la". $\left(\mathrm{CDCl}_{3}\right.$ solvent was used, $\left.400 \mathrm{NMR} \mathrm{MHz}\right)$
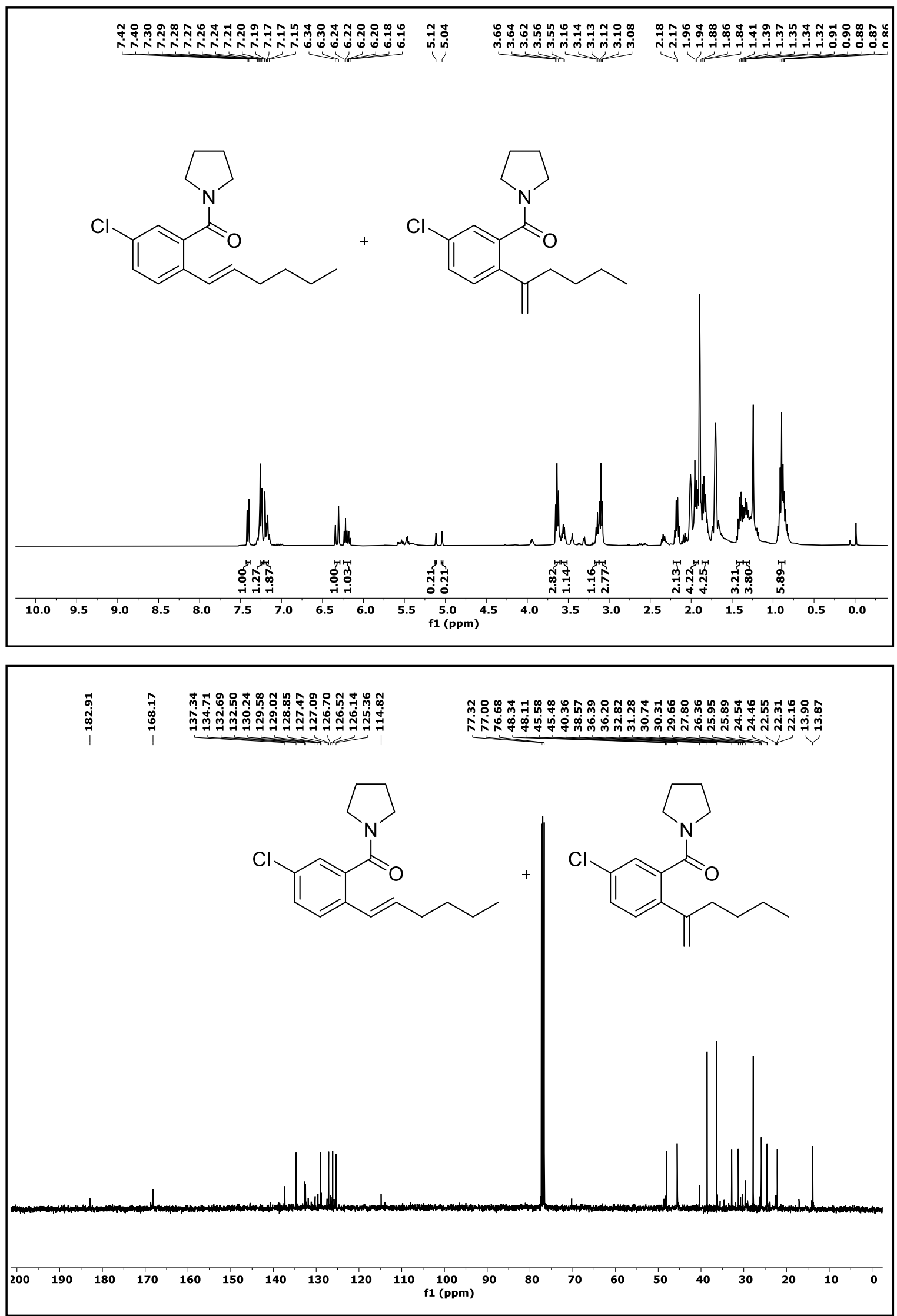
${ }^{1} \mathrm{H}$ and ${ }^{13} \mathrm{C}$ NMR Spectra of Compound 3ma/3ma'.( $\mathrm{CDCl}_{3}$ solvent was used,400 NMR MHz)

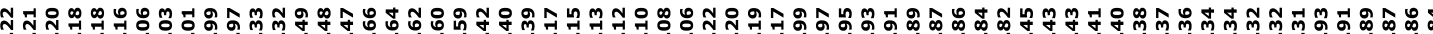
政<smiles>CCCC/C=C/c1ccc(F)cc1C(=O)N1CCCC1</smiles>
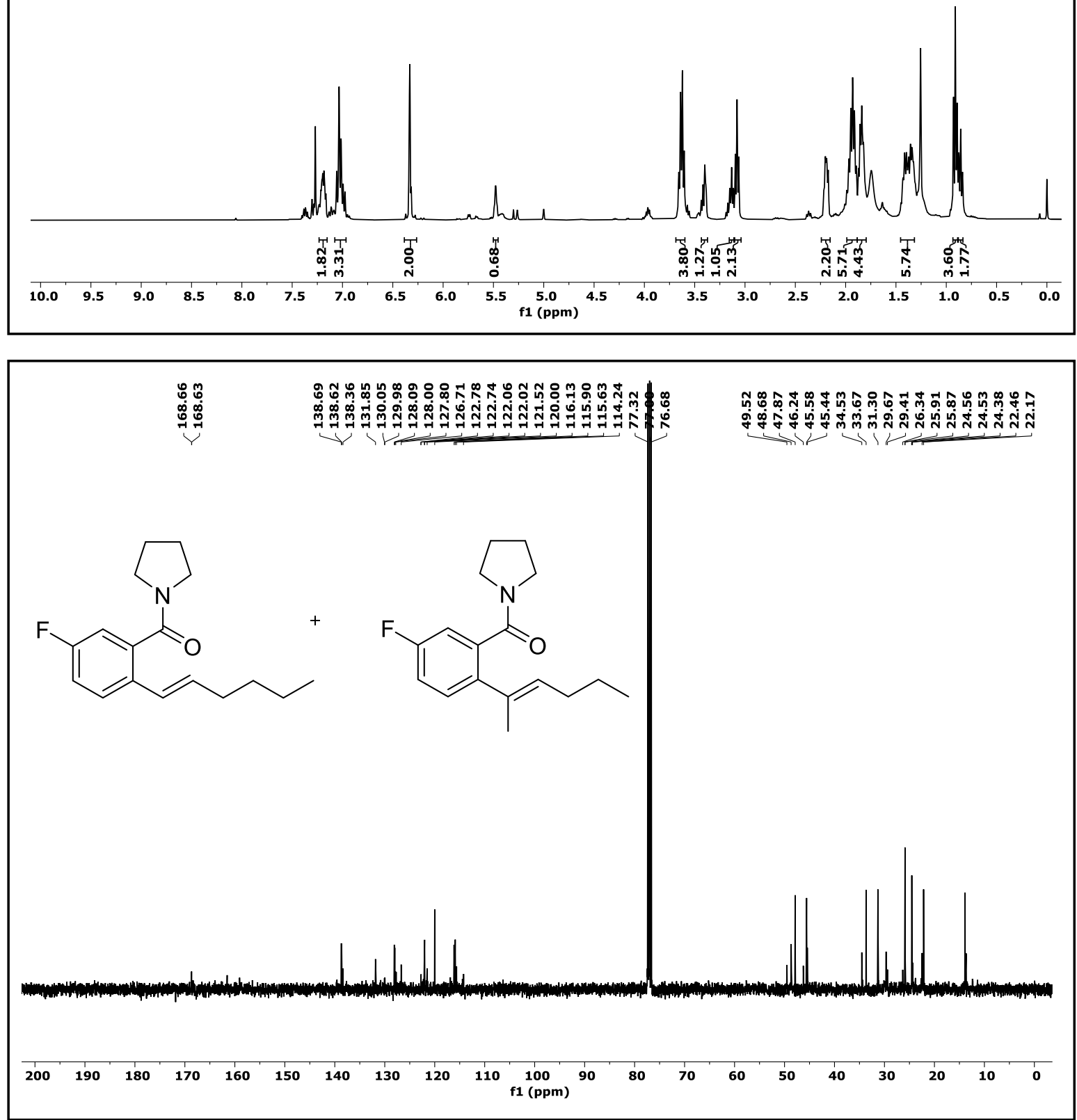
${ }^{1} \mathrm{H}$ and ${ }^{13} \mathrm{C}$ NMR Spectra of Compound 3na/3na'. $\left(\mathrm{CDCl}_{3}\right.$ solvent was used, $\left.400 \mathrm{NMR} \mathrm{MHz}\right)$
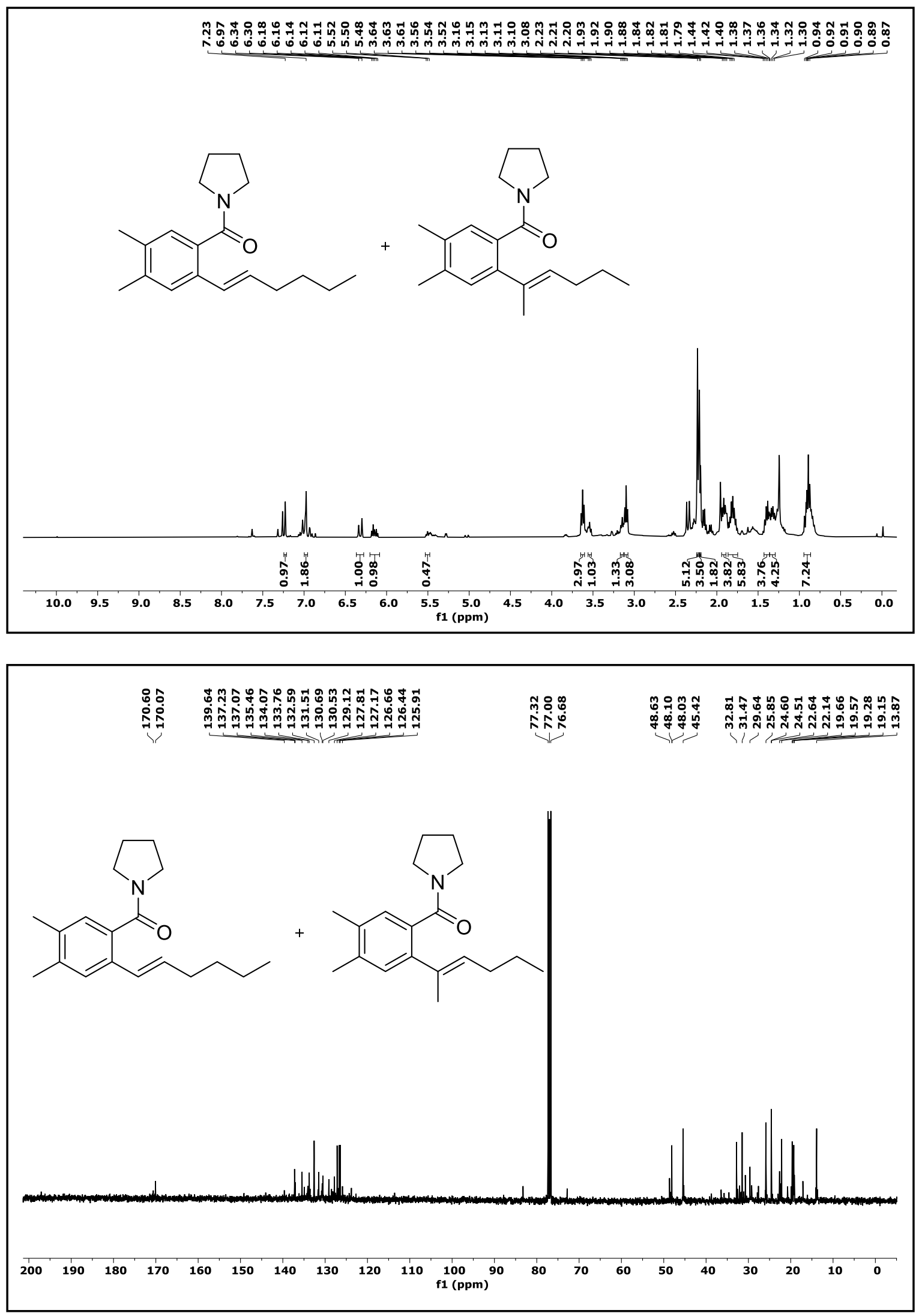
${ }^{1} \mathrm{H}$ and ${ }^{13} \mathrm{C}$ NMR Spectra of Compound 3oa/3oa'. $\left(\mathrm{CDCl}_{3}\right.$ solvent was used, $\left.400 \mathrm{NMR} \mathrm{MHz}\right)$
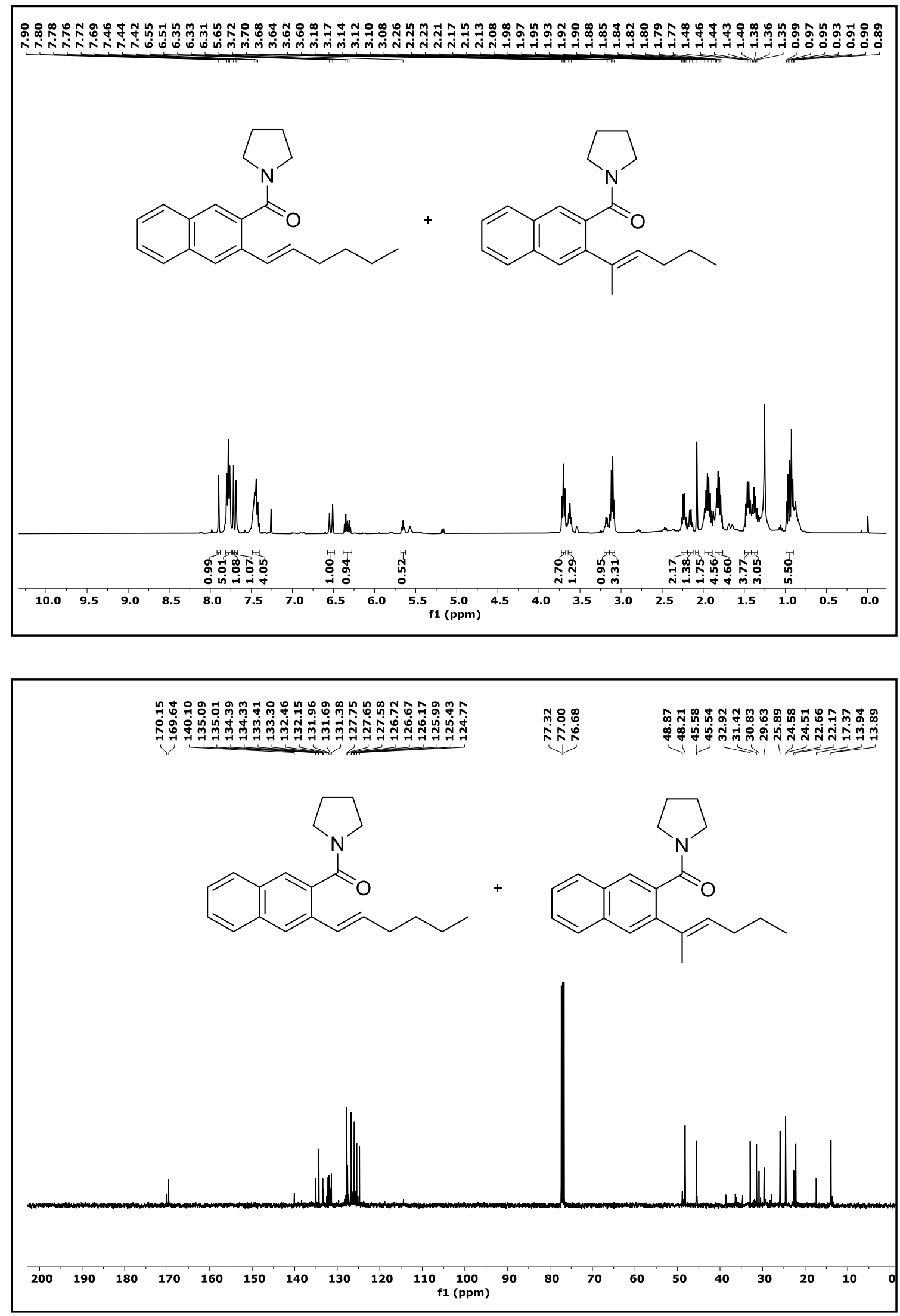
${ }^{1} \mathrm{H}$ and ${ }^{13} \mathrm{C}$ NMR Spectra of Compound 3pa/3pa'. ( $\mathrm{CDCl}_{3}$ solvent was used, $\left.400 \mathrm{NMR} \mathrm{MHz}\right)$
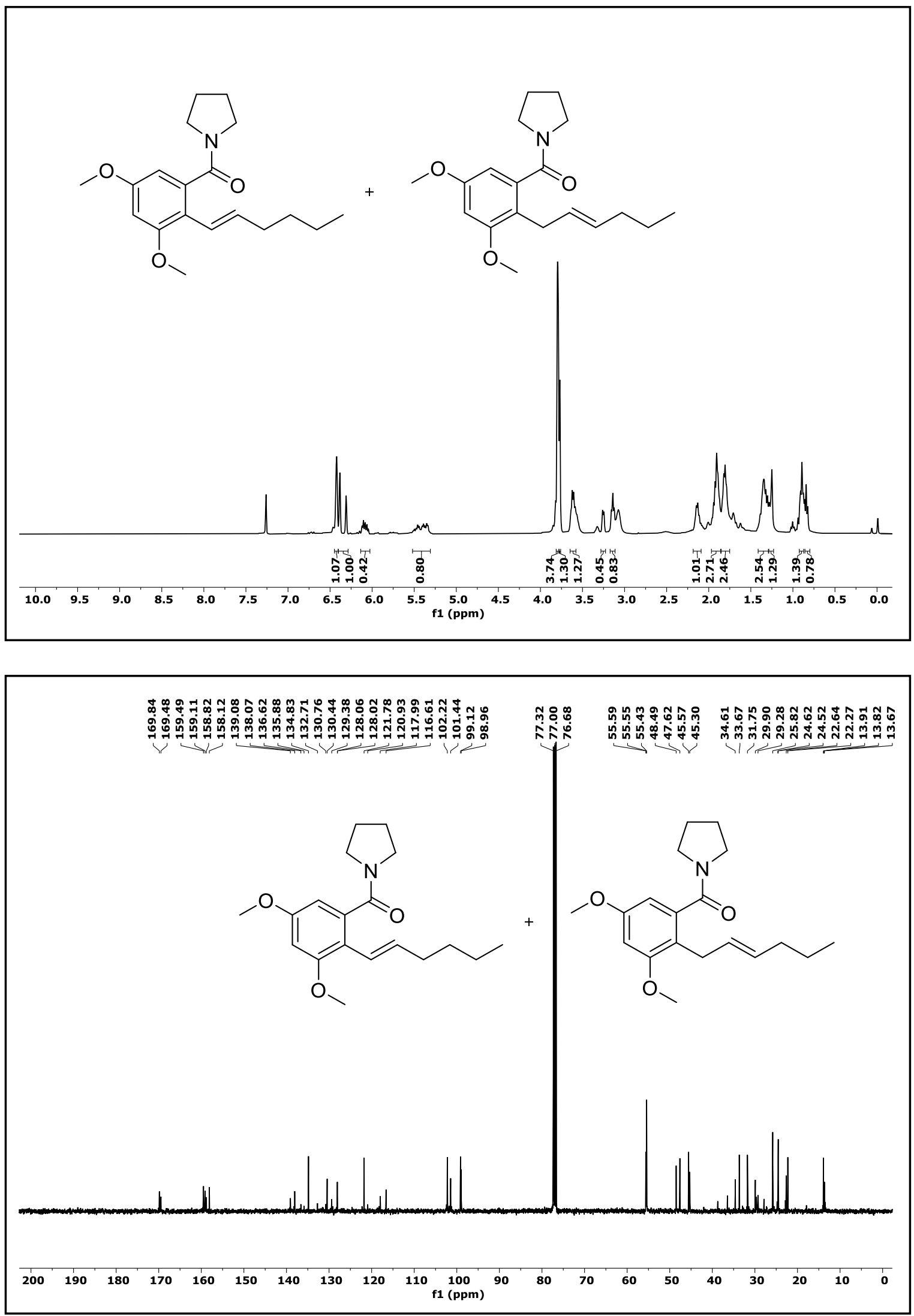
${ }^{1} \mathrm{H}$ and ${ }^{13} \mathrm{C}$ NMR Spectra of Compound 3qa/3qa'. $\left(\mathrm{CDCl}_{3}\right.$ solvent was used, $\left.400 \mathrm{NMR} \mathrm{MHz}\right)$
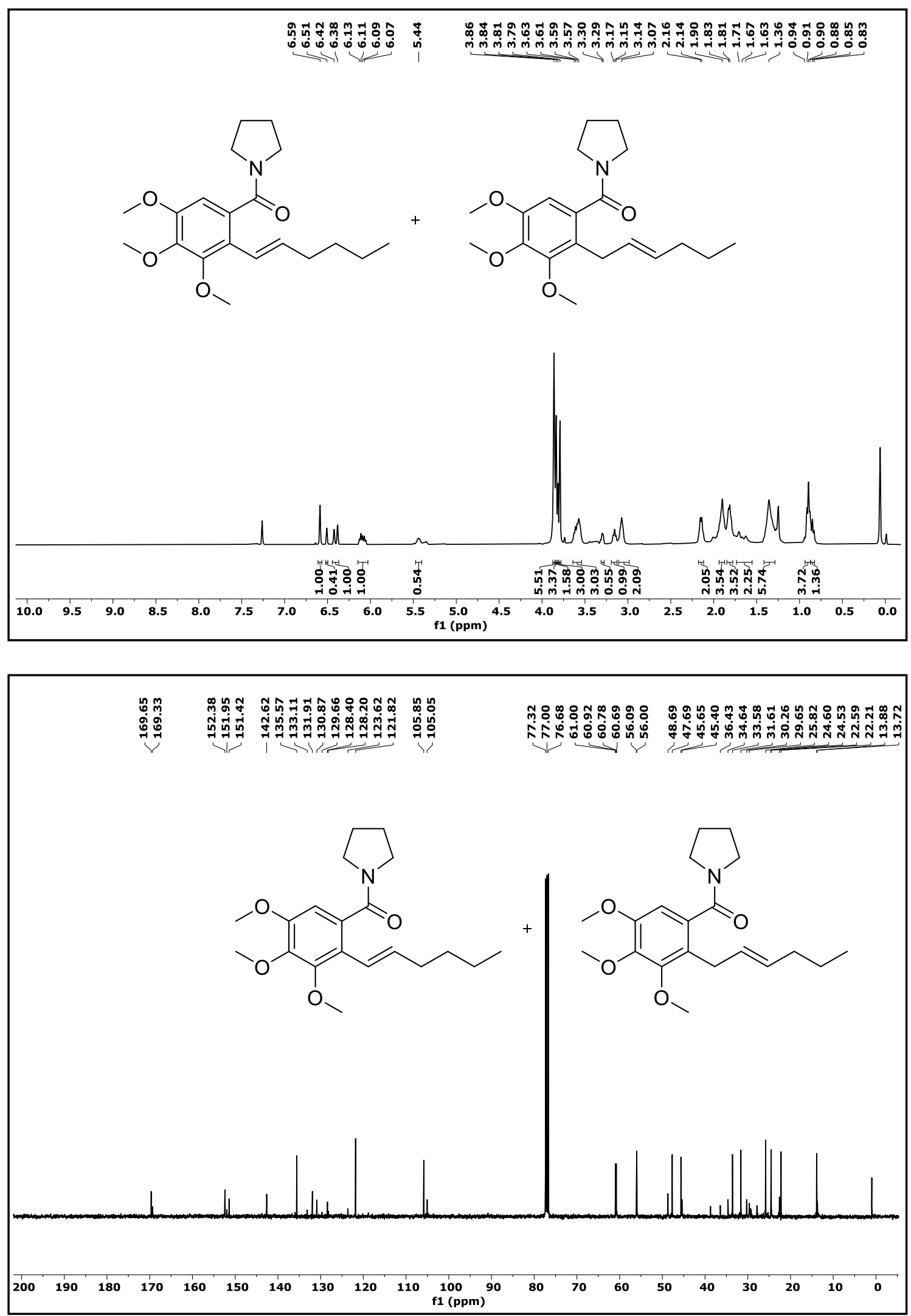
${ }^{1} \mathrm{H}$ and ${ }^{13} \mathrm{C}$ NMR Spectra of Compound 3ra. $\left(\mathrm{CDCl}_{3}\right.$ solvent was used, $\left.400 \mathrm{NMR} \mathrm{MHz}\right)$
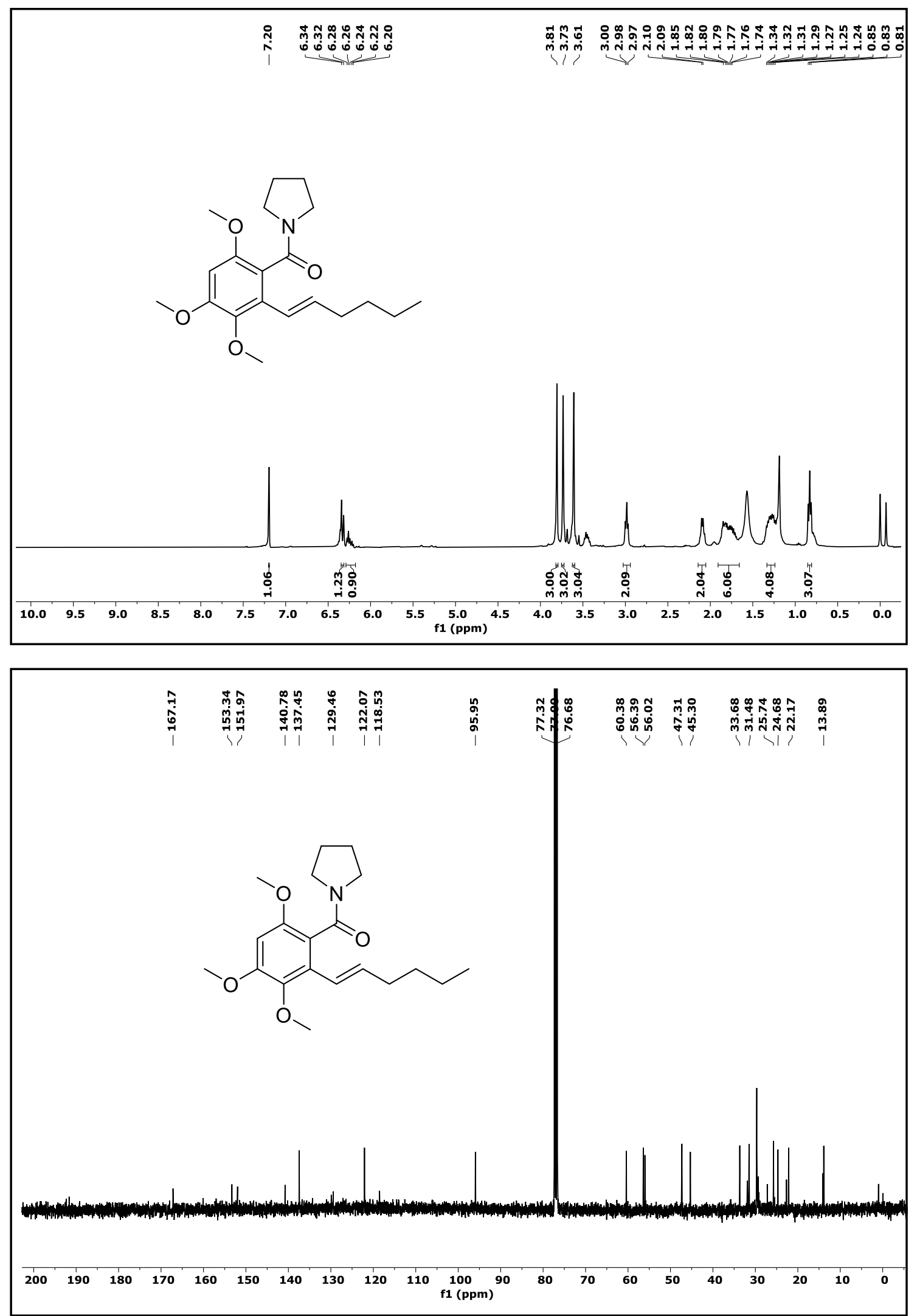
${ }^{1} \mathrm{H}$ and ${ }^{13} \mathrm{C}$ NMR Spectra of Compound 3sa. $\left(\mathrm{CDCl}_{3}\right.$ solvent was used, $\left.500 \mathrm{NMR} \mathrm{MHz}\right)$
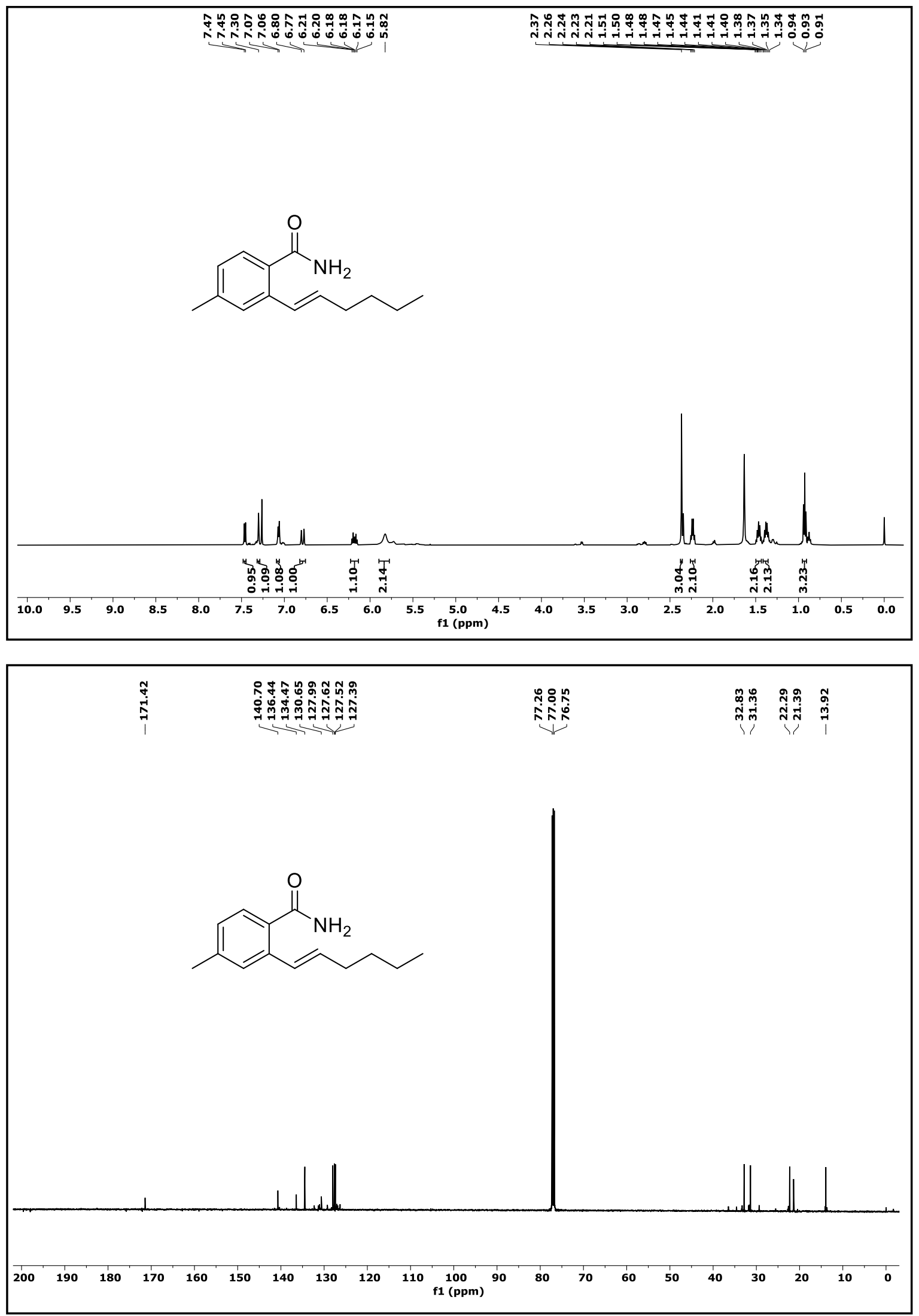
${ }^{1} \mathrm{H}$ and ${ }^{13} \mathrm{C}$ NMR Spectra of Compound 3ta. $\left(\mathrm{CDCl}_{3}\right.$ solvent was used, $\left.500 \mathrm{NMR} \mathrm{MHz}\right)$
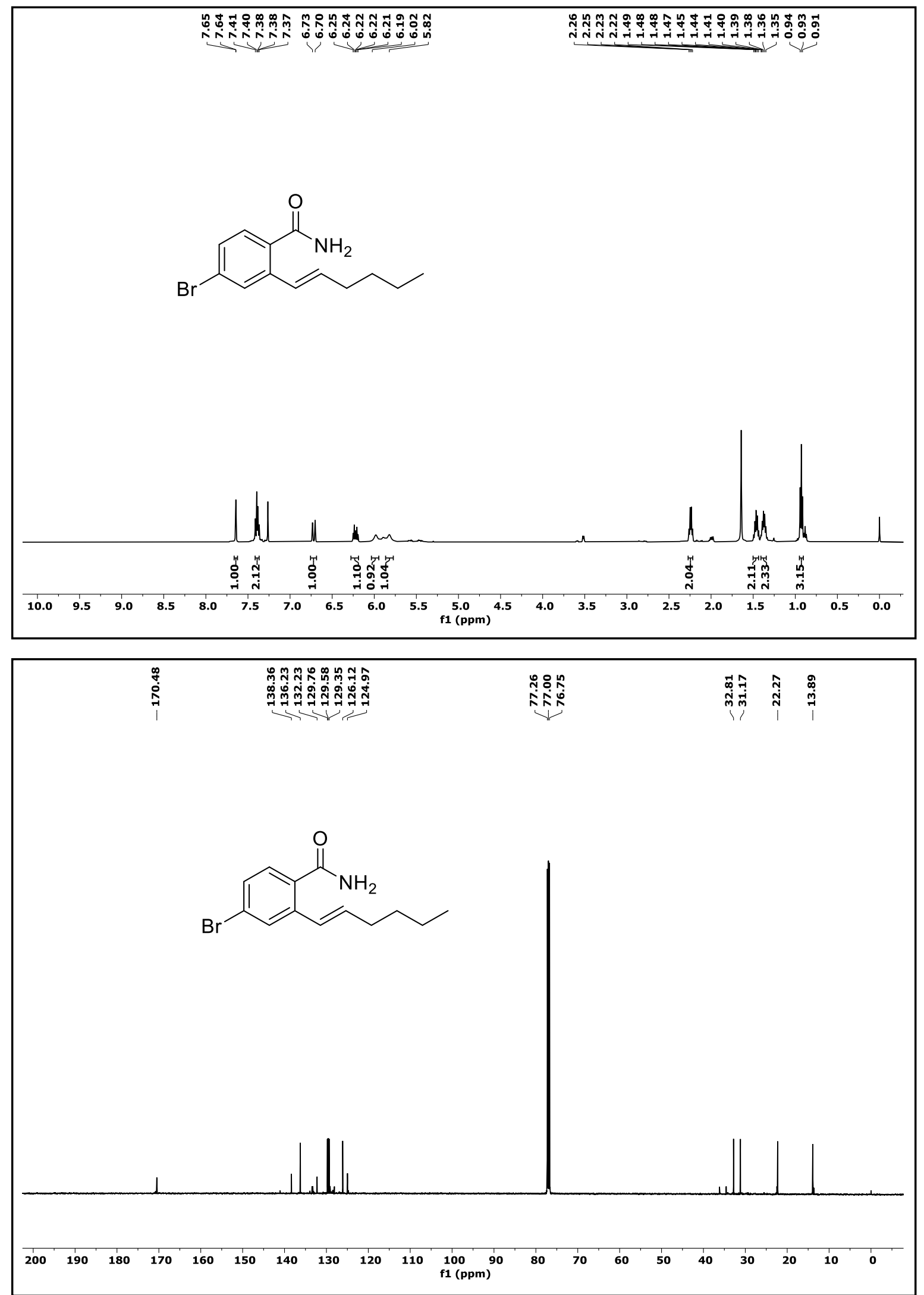
${ }^{1} \mathrm{H}$ and ${ }^{13} \mathrm{C}$ NMR Spectra of Compound 3ua/3ua". $\left(\mathrm{CDCl}_{3}\right.$ solvent was used,400 NMR MHz)

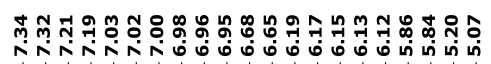

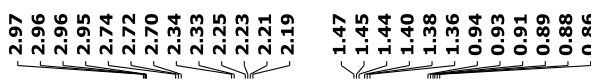<smiles>C=C(CCCC)c1cc(C)ccc1C(=O)NC</smiles>

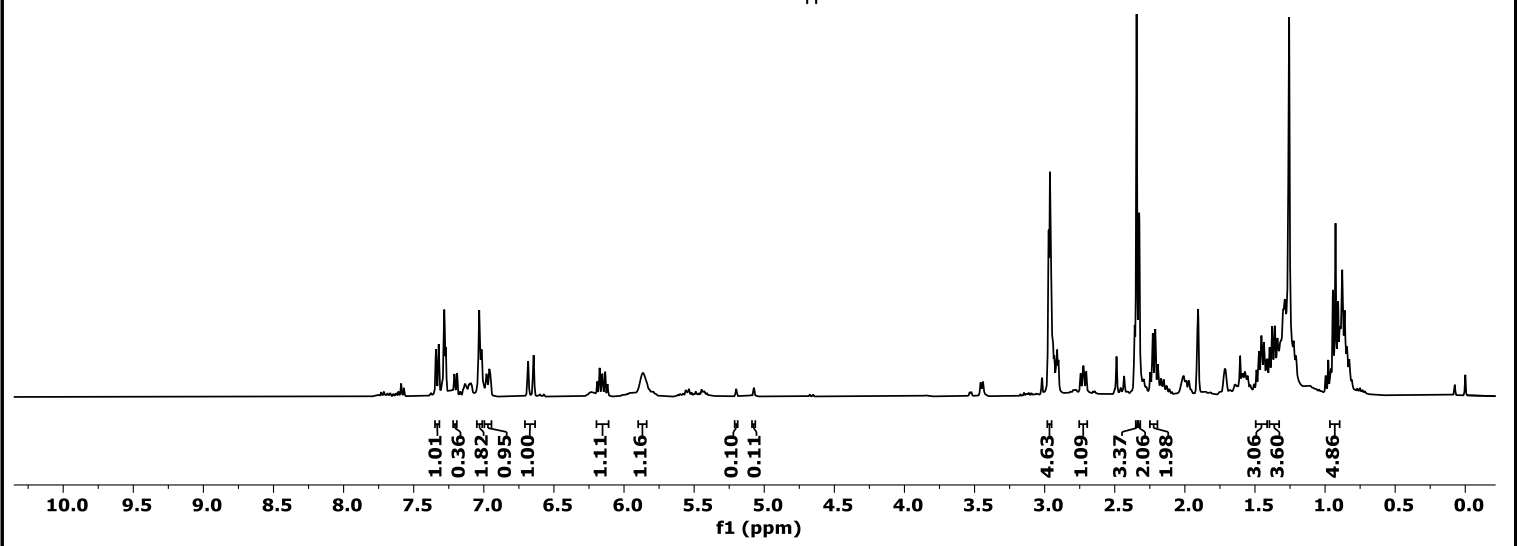

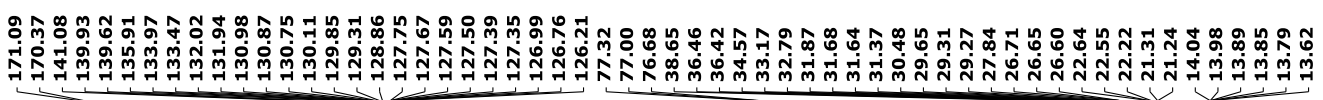

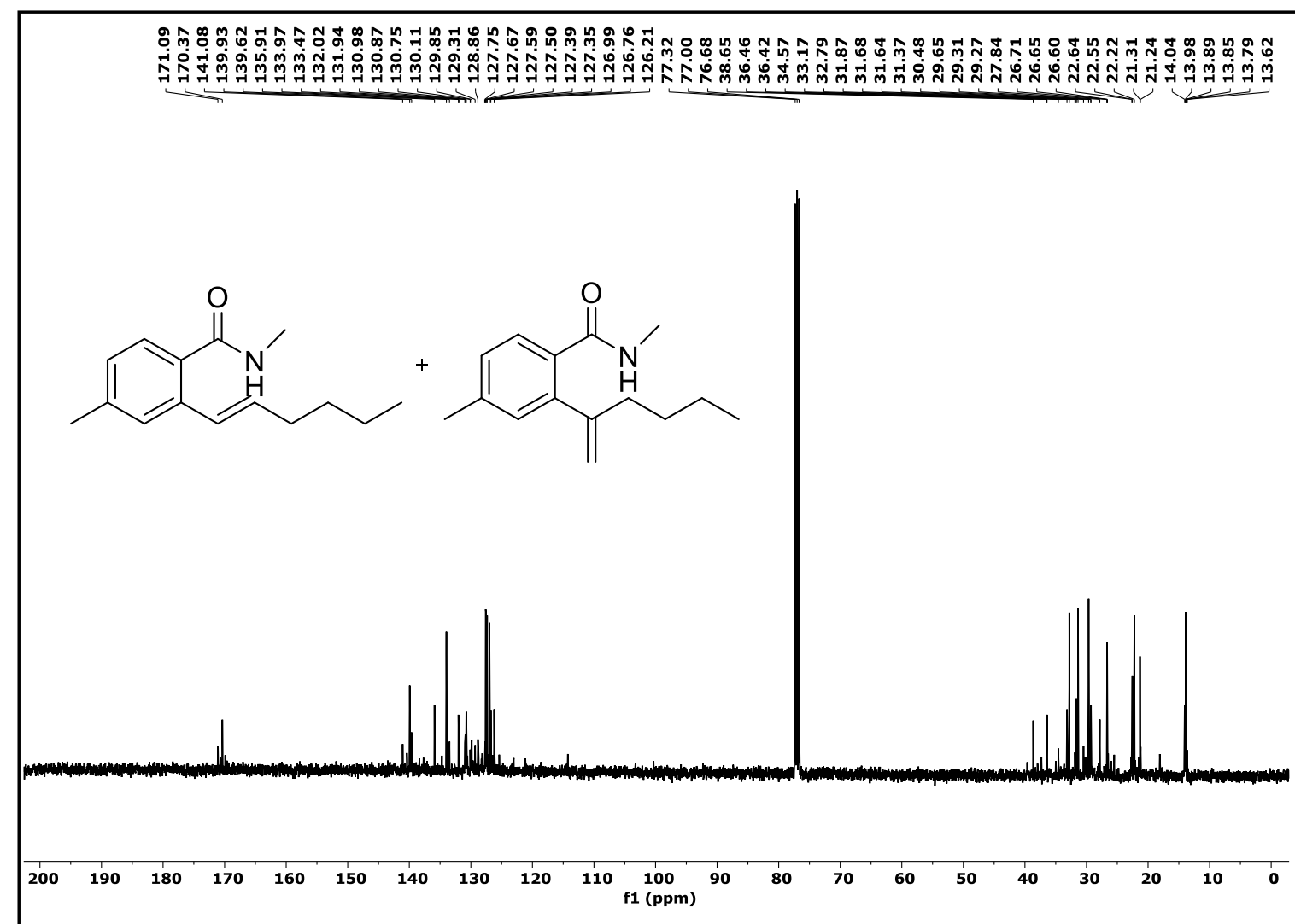

S53 
${ }^{1} \mathrm{H}$ and ${ }^{13} \mathrm{C}$ NMR Spectra of Compound 4ab. $\left(\mathrm{CDCl}_{3}\right.$ solvent was used, $\left.400 \mathrm{NMR} \mathrm{MHz}\right)$
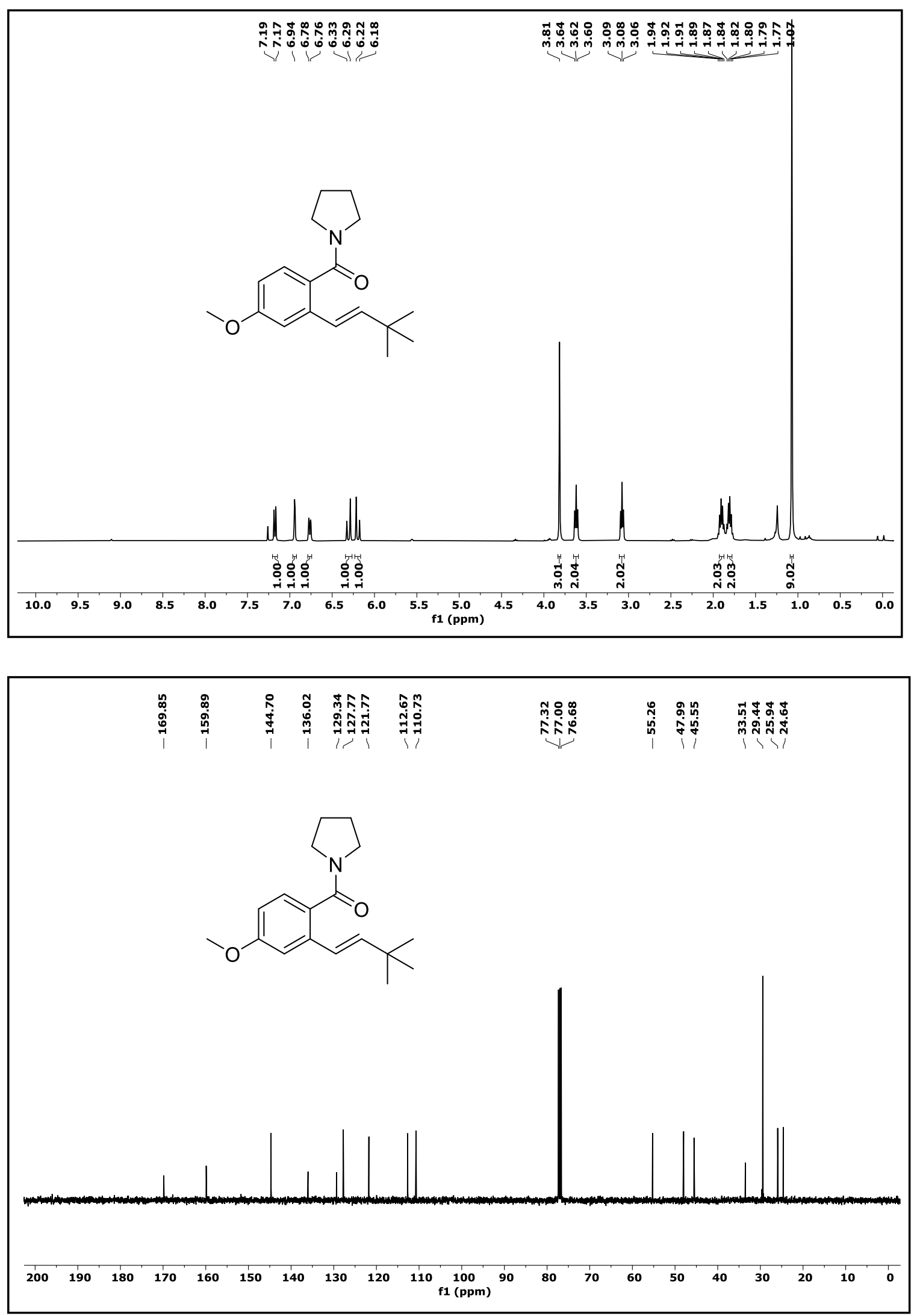
${ }^{1} \mathrm{H}$ and ${ }^{13} \mathrm{C}$ NMR Spectra of Compound $4 \mathbf{b b} .\left(\mathrm{CDCl}_{3}\right.$ solvent was used, $\left.500 \mathrm{NMR} \mathrm{MHz}\right)$
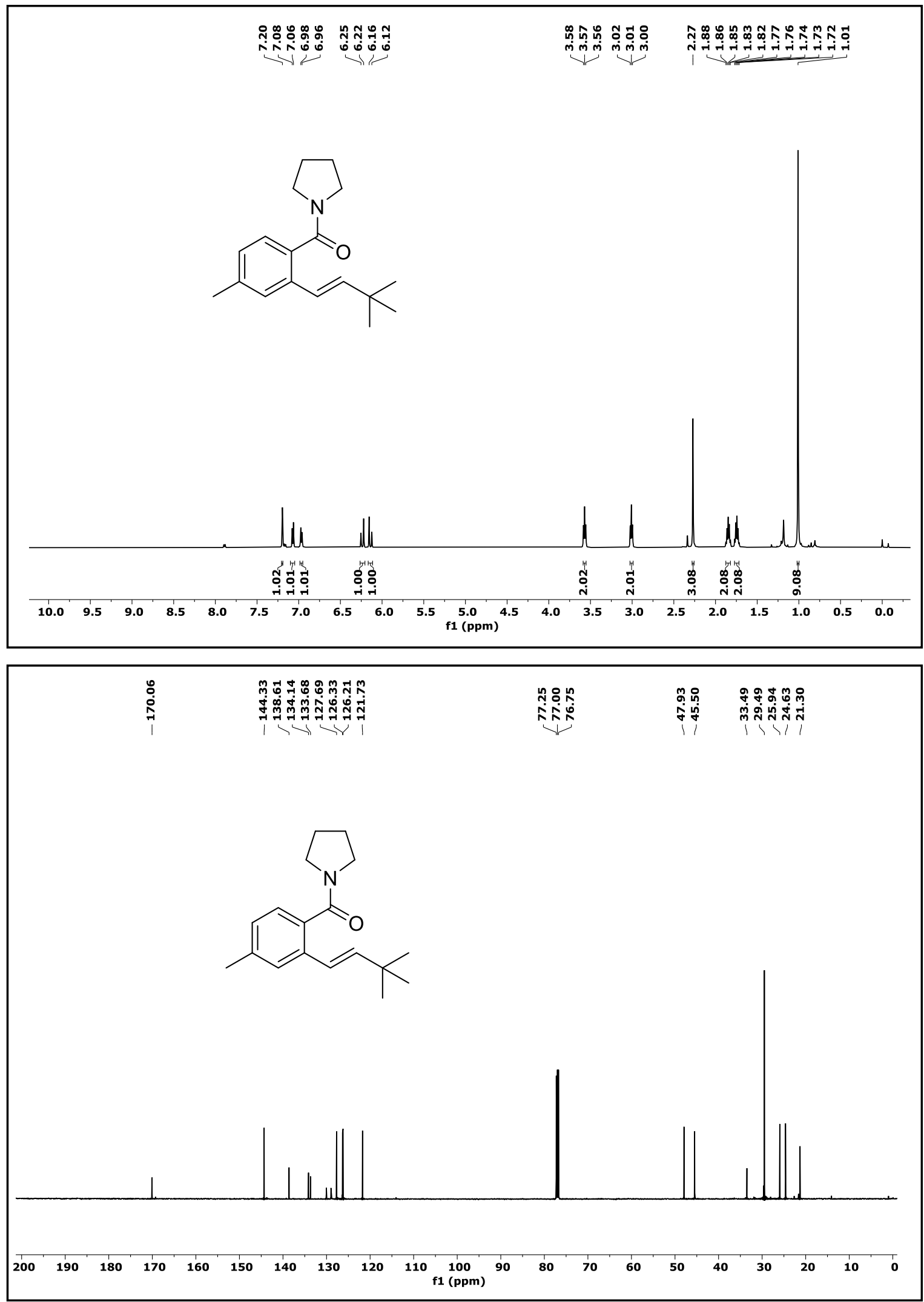
${ }^{1} \mathrm{H}$ and ${ }^{13} \mathrm{C}$ NMR Spectra of Compound 4cb. $\left(\mathrm{CDCl}_{3}\right.$ solvent was used, $\left.500 \mathrm{NMR} \mathrm{MHz}\right)$
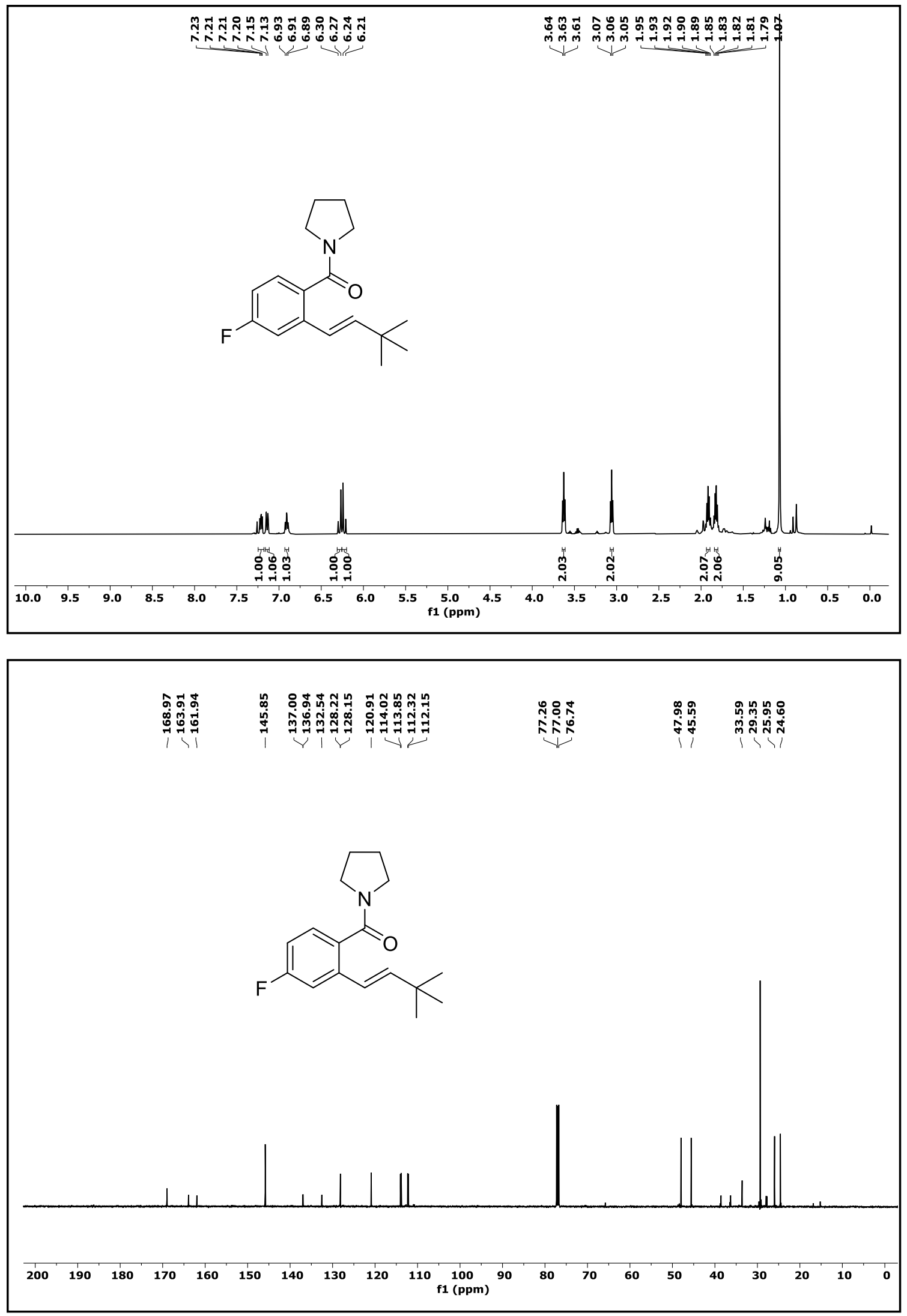
${ }^{1} \mathrm{H}$ and ${ }^{13} \mathrm{C}$ NMR Spectra of Compound 4eb. $\left(\mathrm{CDCl}_{3}\right.$ solvent was used, $\left.400 \mathrm{NMR} \mathrm{MHz}\right)$
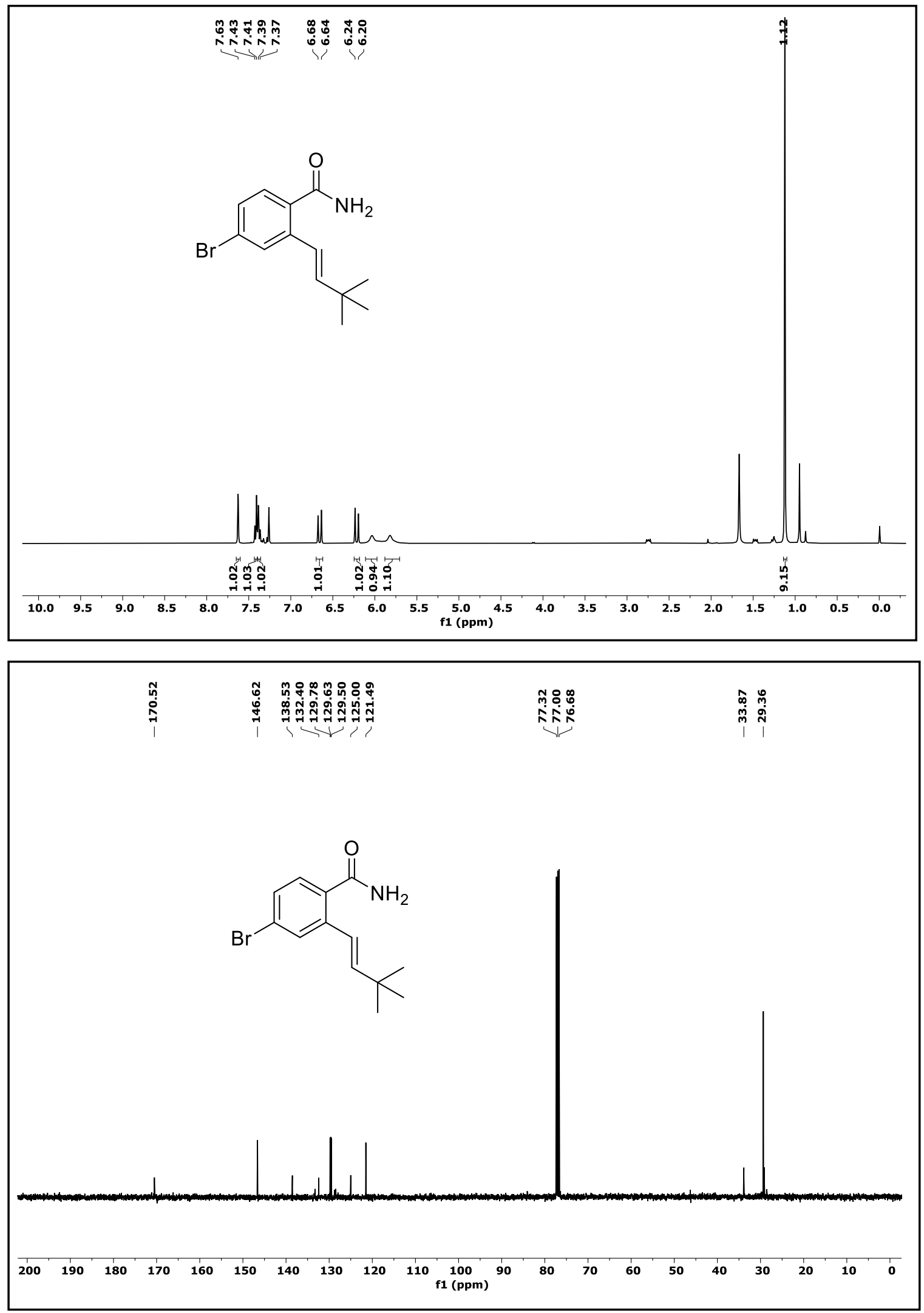
${ }^{1} \mathrm{H}$ and ${ }^{13} \mathrm{C}$ NMR Spectra of Compound 4bc. $\left(\mathrm{CDCl}_{3}\right.$ solvent was used, $\left.400 \mathrm{NMR} \mathrm{MHz}\right)$
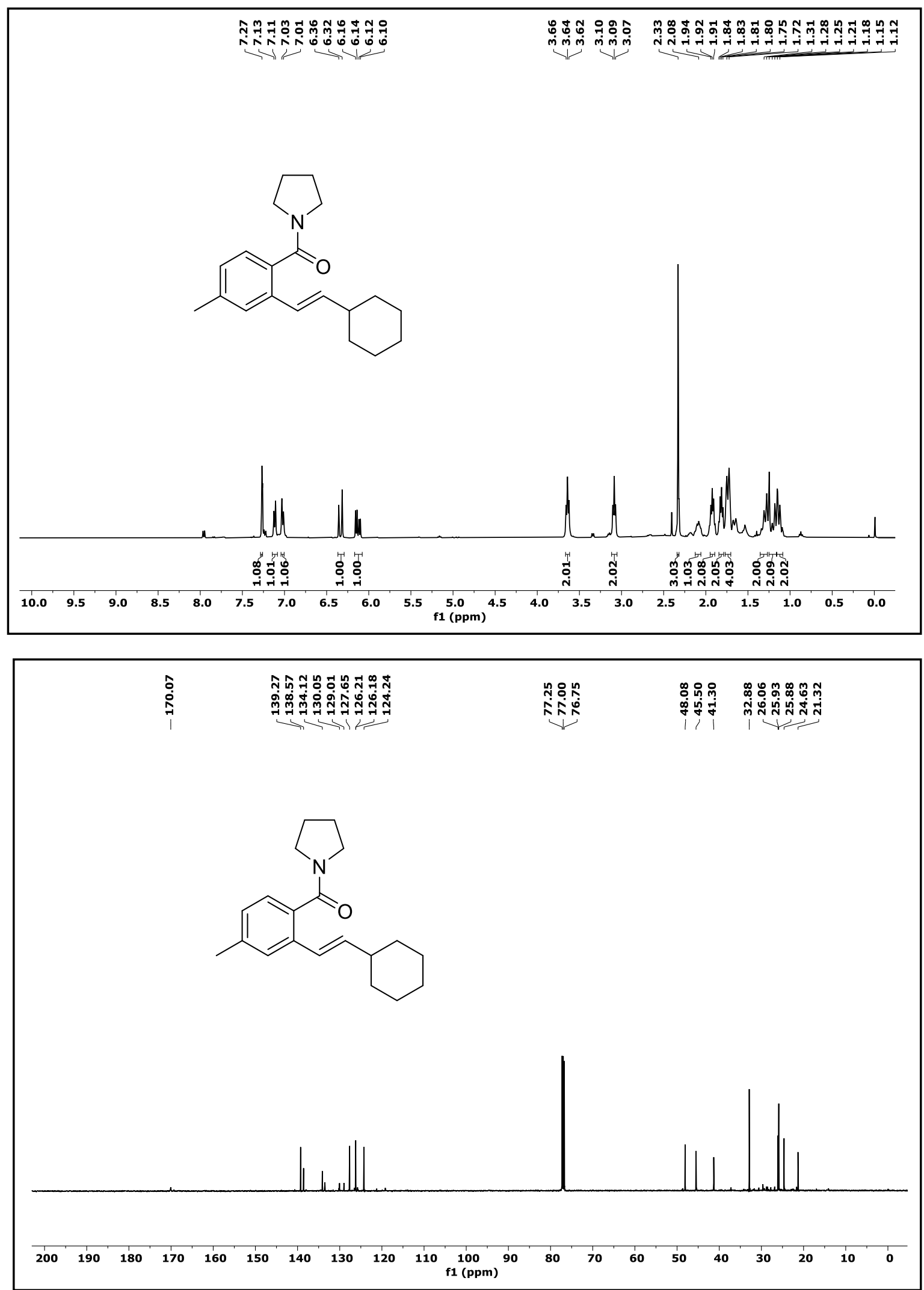
${ }^{1} \mathrm{H}$ and ${ }^{13} \mathrm{C}$ NMR Spectra of Compound 4vc. $\left(\mathrm{CDCl}_{3}\right.$ solvent was used, $\left.400 \mathrm{NMR} \mathrm{MHz}\right)$
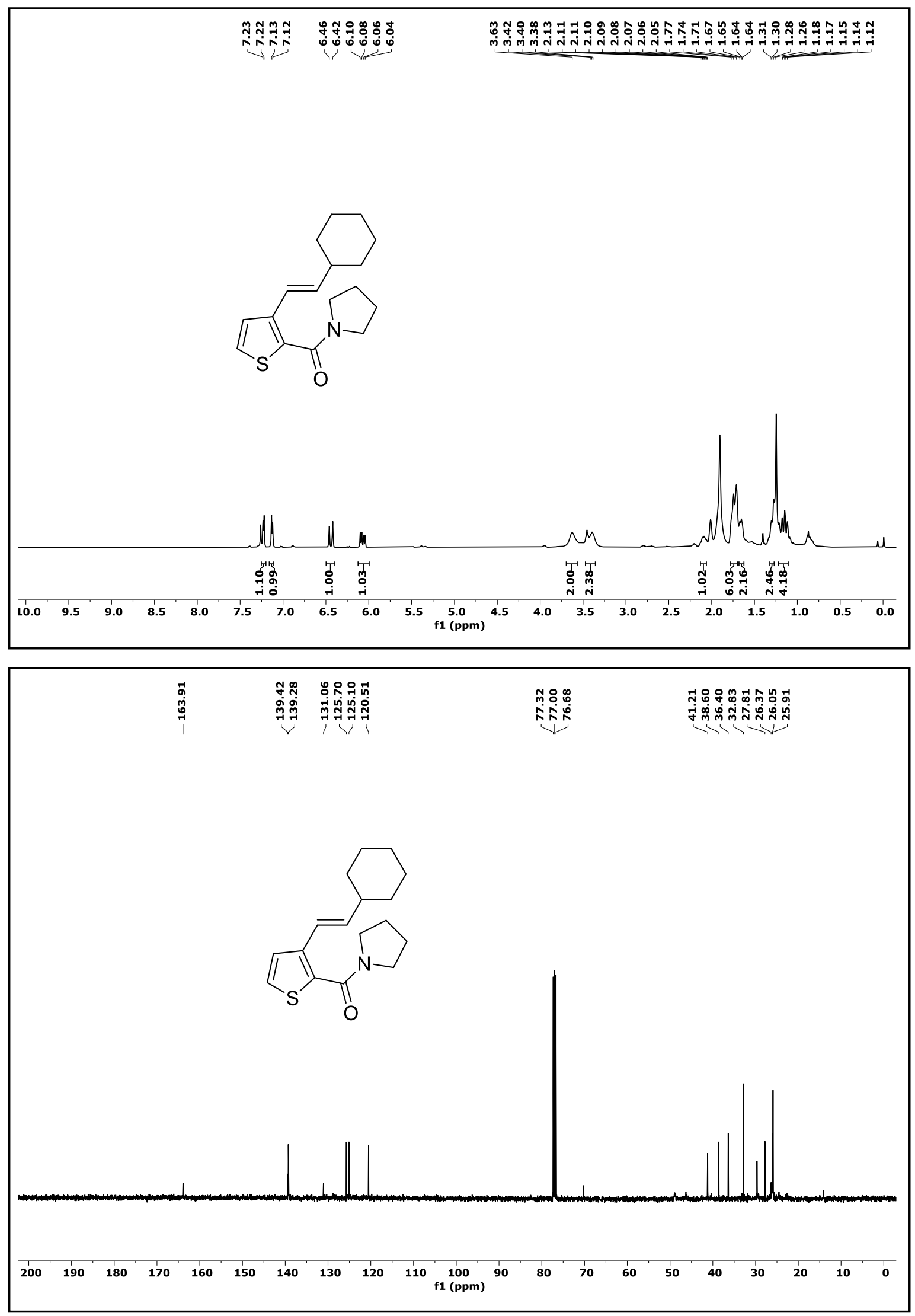
${ }^{1} \mathrm{H}$ and ${ }^{13} \mathrm{C}$ NMR Spectra of Compound 4ad. $\left(\mathrm{CDCl}_{3}\right.$ solvent was used, $\left.400 \mathrm{NMR} \mathrm{MHz}\right)$
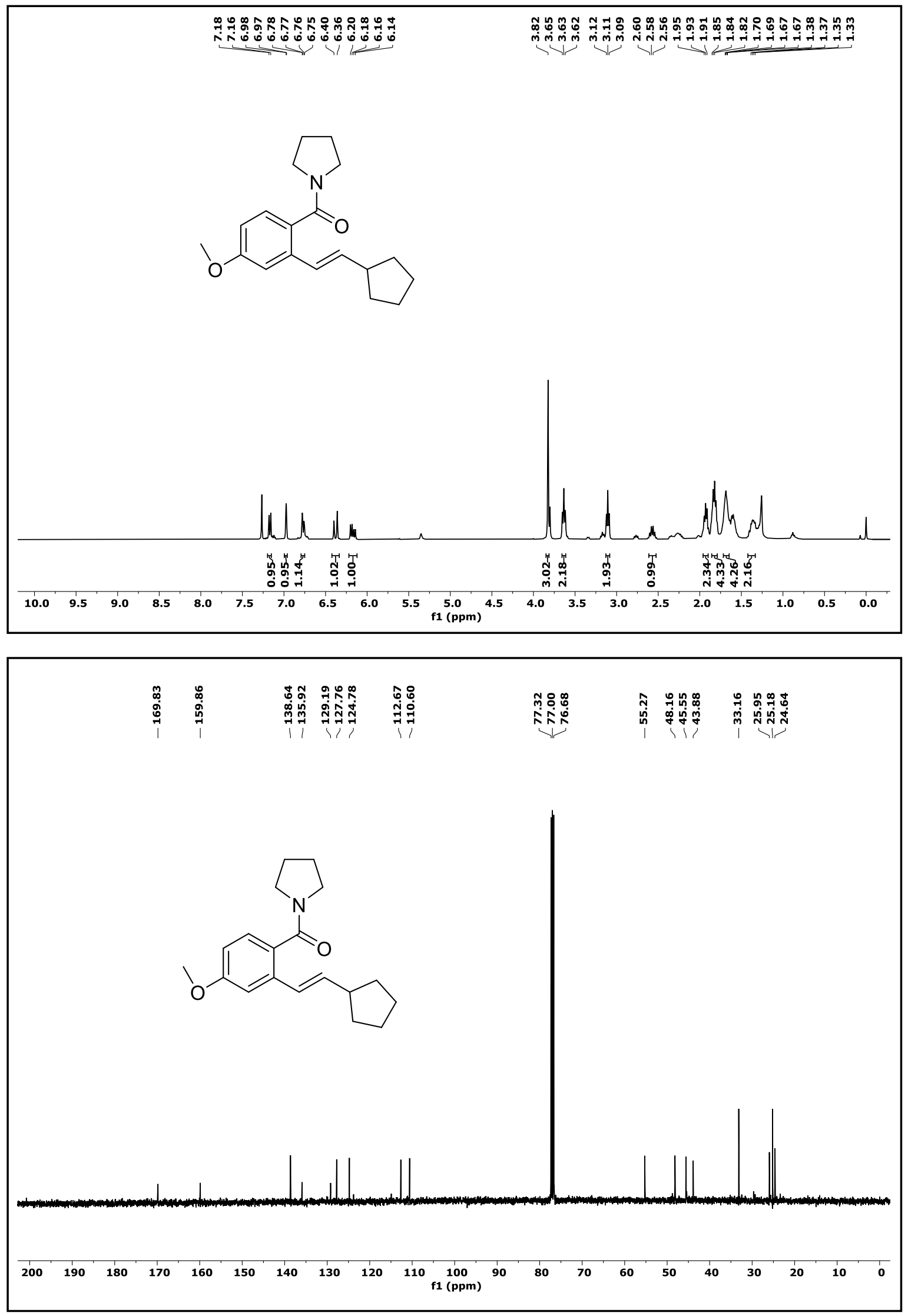
${ }^{1} \mathrm{H}$ and ${ }^{13} \mathrm{C}$ NMR Spectra of Compound 4oe/4oe'. $\left(\mathrm{CDCl}_{3}\right.$ solvent was used, $\left.400 \mathrm{NMR} \mathrm{MHz}\right)$
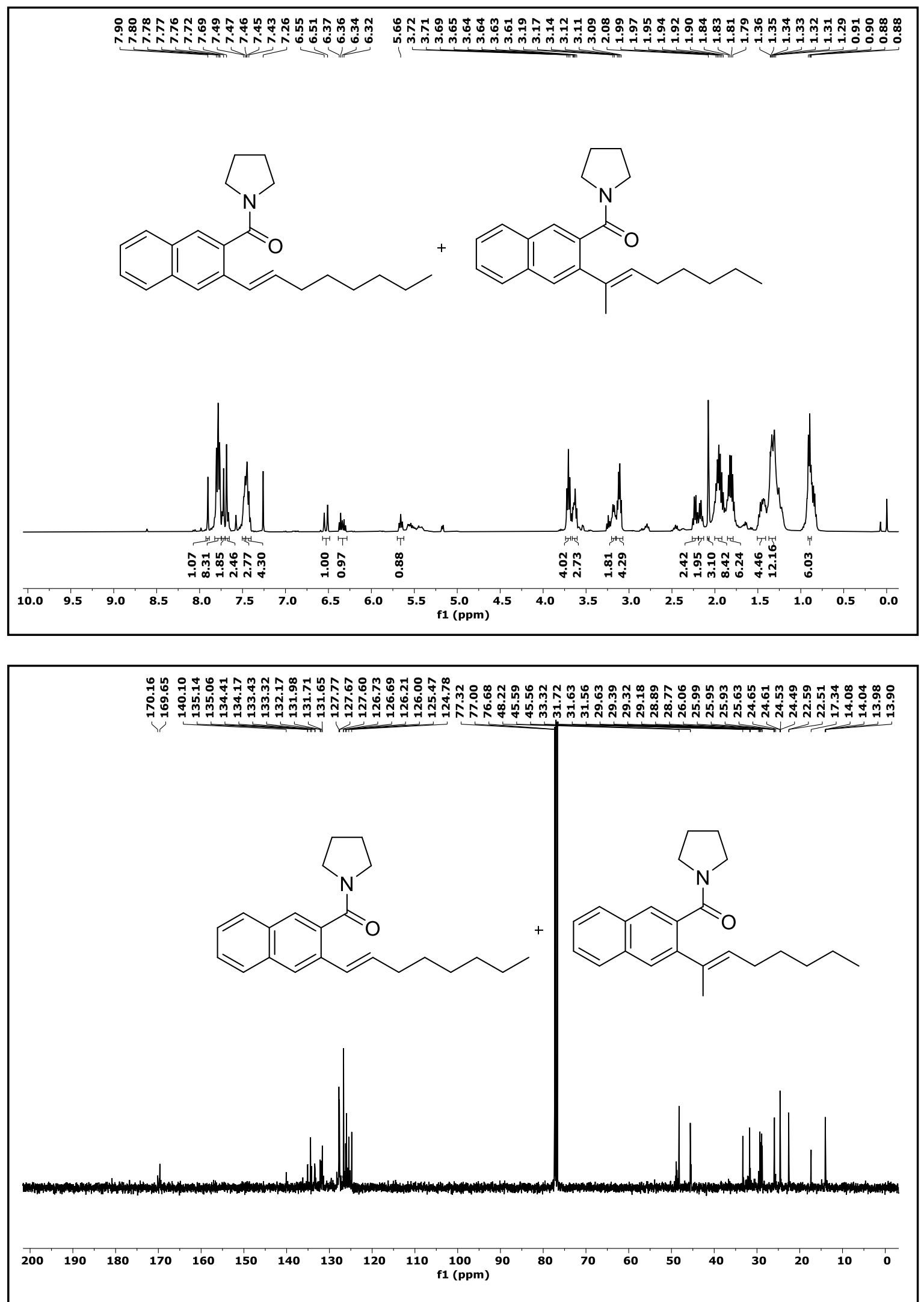
${ }^{1} \mathrm{H}$ and ${ }^{13} \mathrm{C}$ NMR Spectra of Compound 4of/4of'. $\left(\mathrm{CDCl}_{3}\right.$ solvent was used, $\left.400 \mathrm{NMR} \mathrm{MHz}\right)$

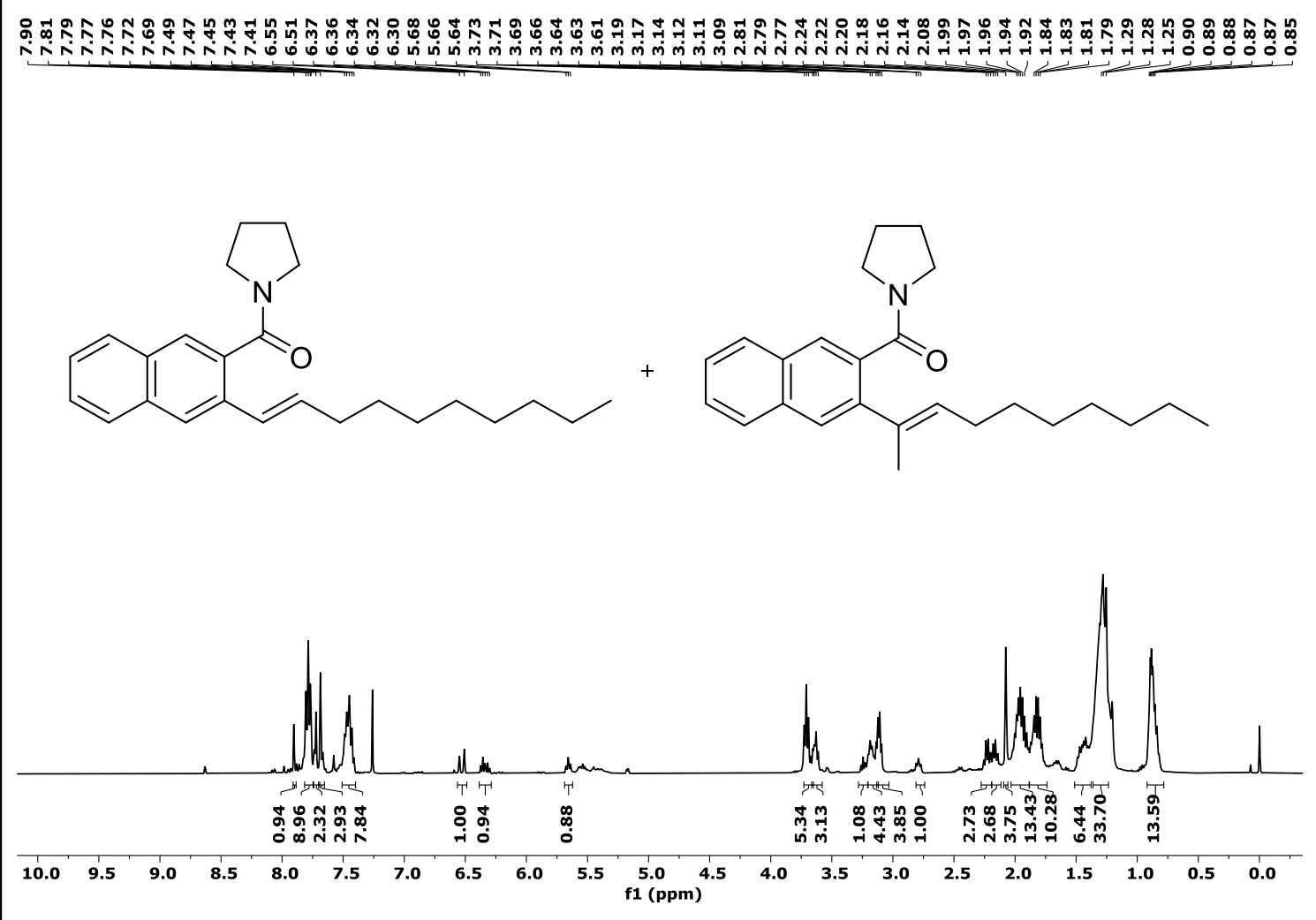

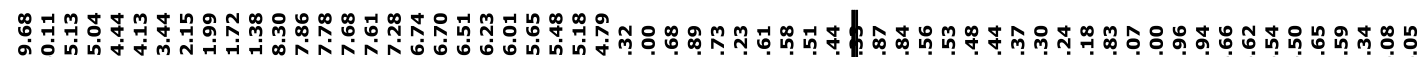

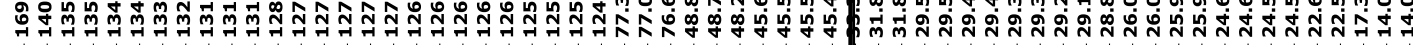

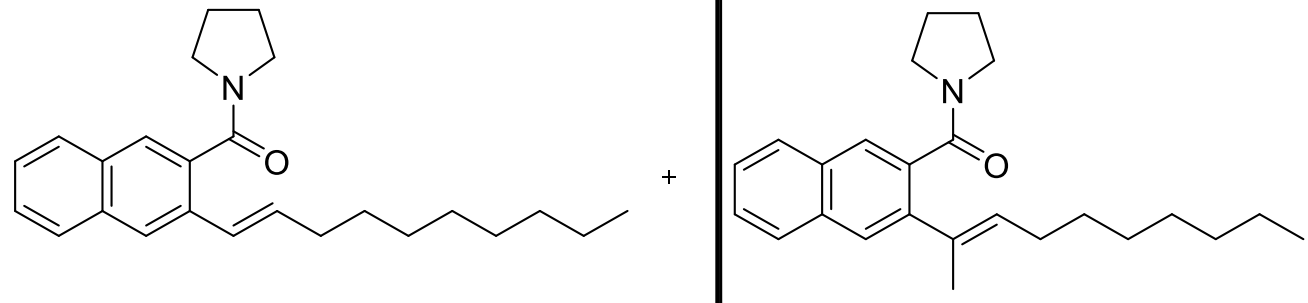

\begin{tabular}{lllllllllllllllllllllllll}
\hline 200 & 190 & 180 & 170 & 160 & 150 & 140 & 130 & 120 & 110 & $\begin{array}{c}100 \\
\mathrm{f1}(\mathrm{ppm})\end{array}$ & 90 & 80 & 70 & 60 & 50 & 40 & 30 & 20 & 10 & 0
\end{tabular} 
${ }^{1} \mathrm{H}$ and ${ }^{13} \mathrm{C}$ NMR Spectra of Compound 4we. $\left(\mathrm{CDCl}_{3}\right.$ solvent was used, $\left.400 \mathrm{NMR} \mathrm{MHz}\right)$
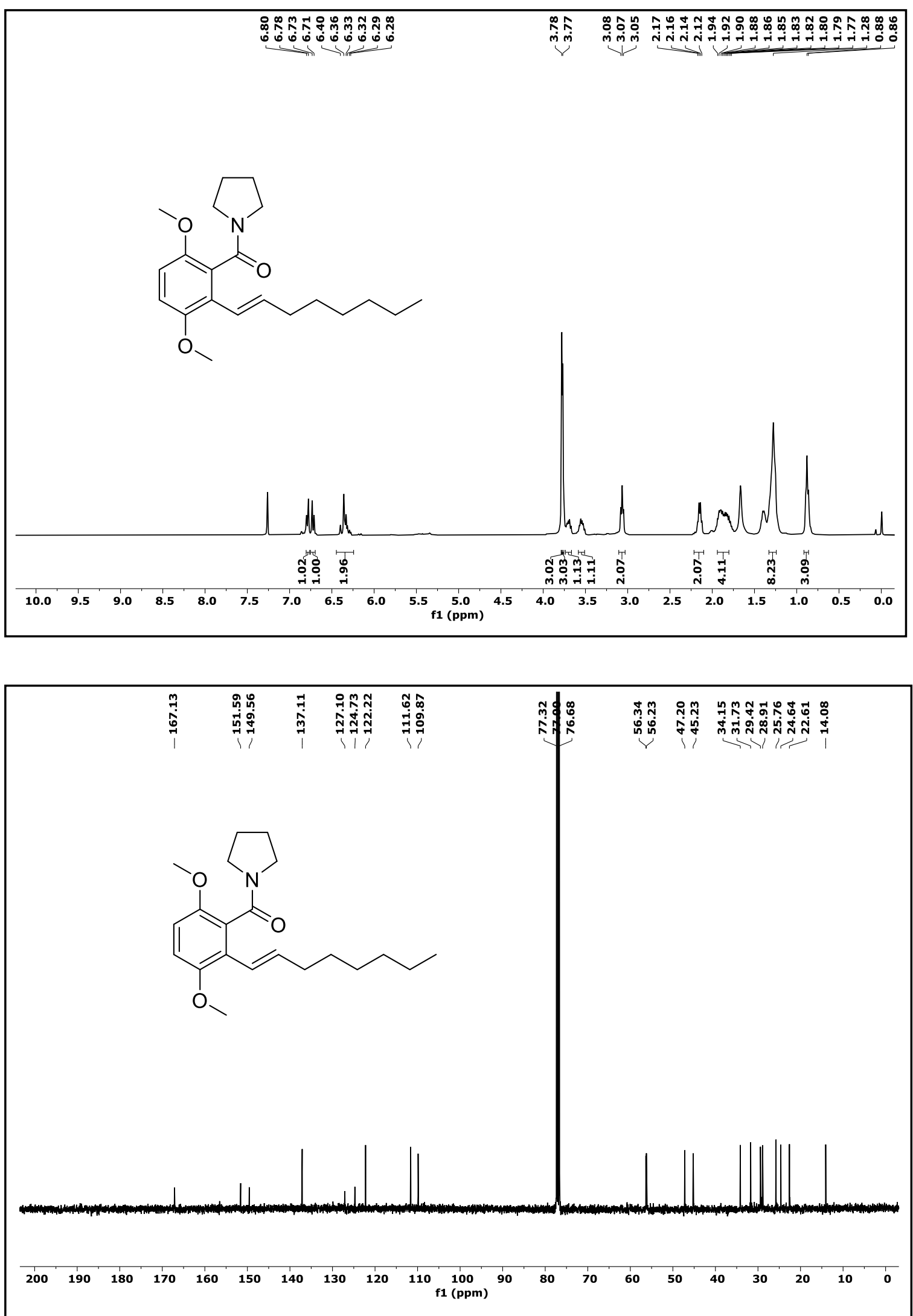
${ }^{1} \mathrm{H}$ and ${ }^{13} \mathrm{C}$ NMR Spectra of Compound 5og/5og'. $\left(\mathrm{CDCl}_{3}\right.$ solvent was used, $\left.400 \mathrm{NMR} \mathrm{MHz}\right)$
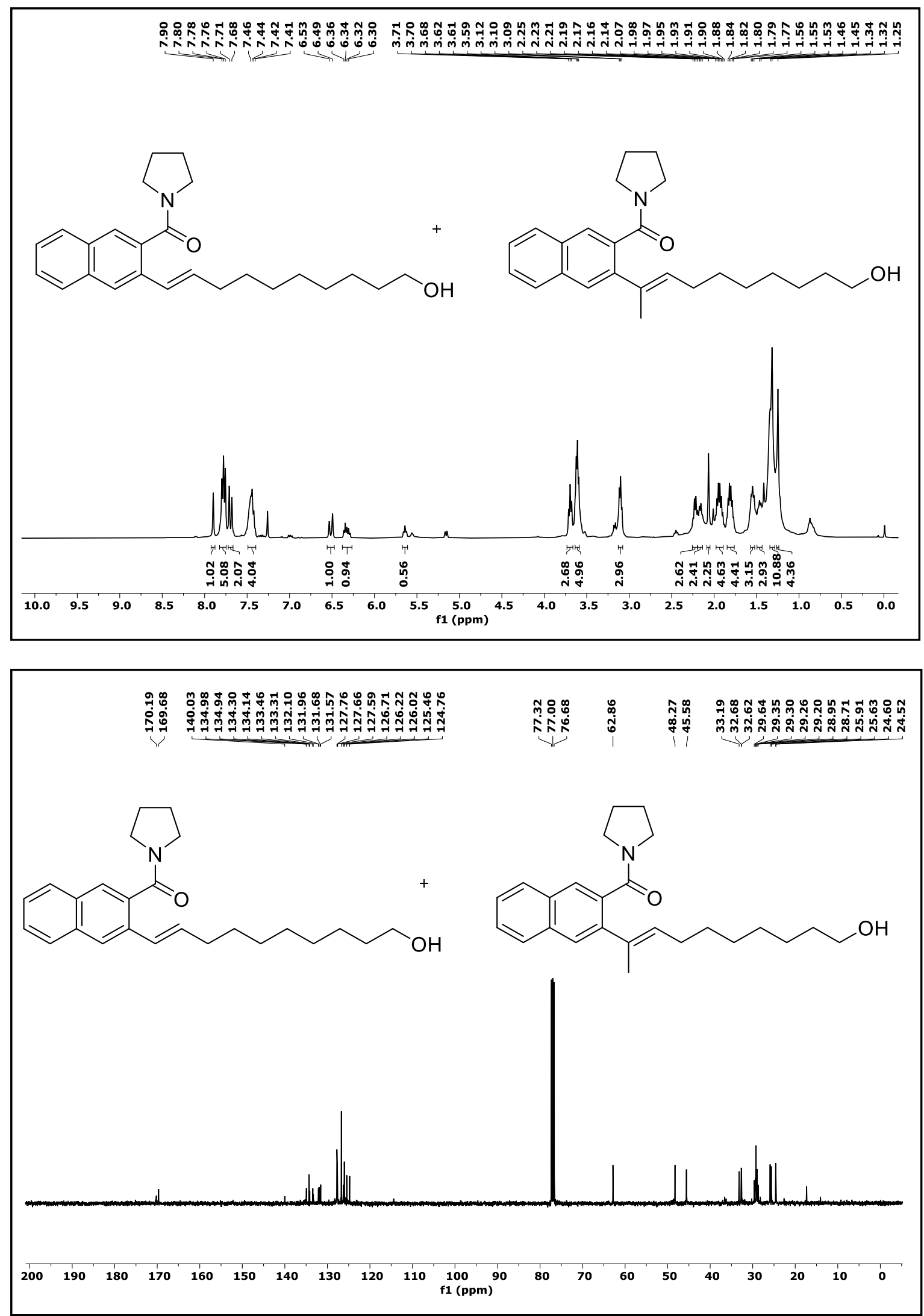
${ }^{1} \mathrm{H}$ and ${ }^{13} \mathrm{C}$ NMR Spectra of Compound 5wg. $\left(\mathrm{CDCl}_{3}\right.$ solvent was used, $\left.400 \mathrm{NMR} \mathrm{MHz}\right)$
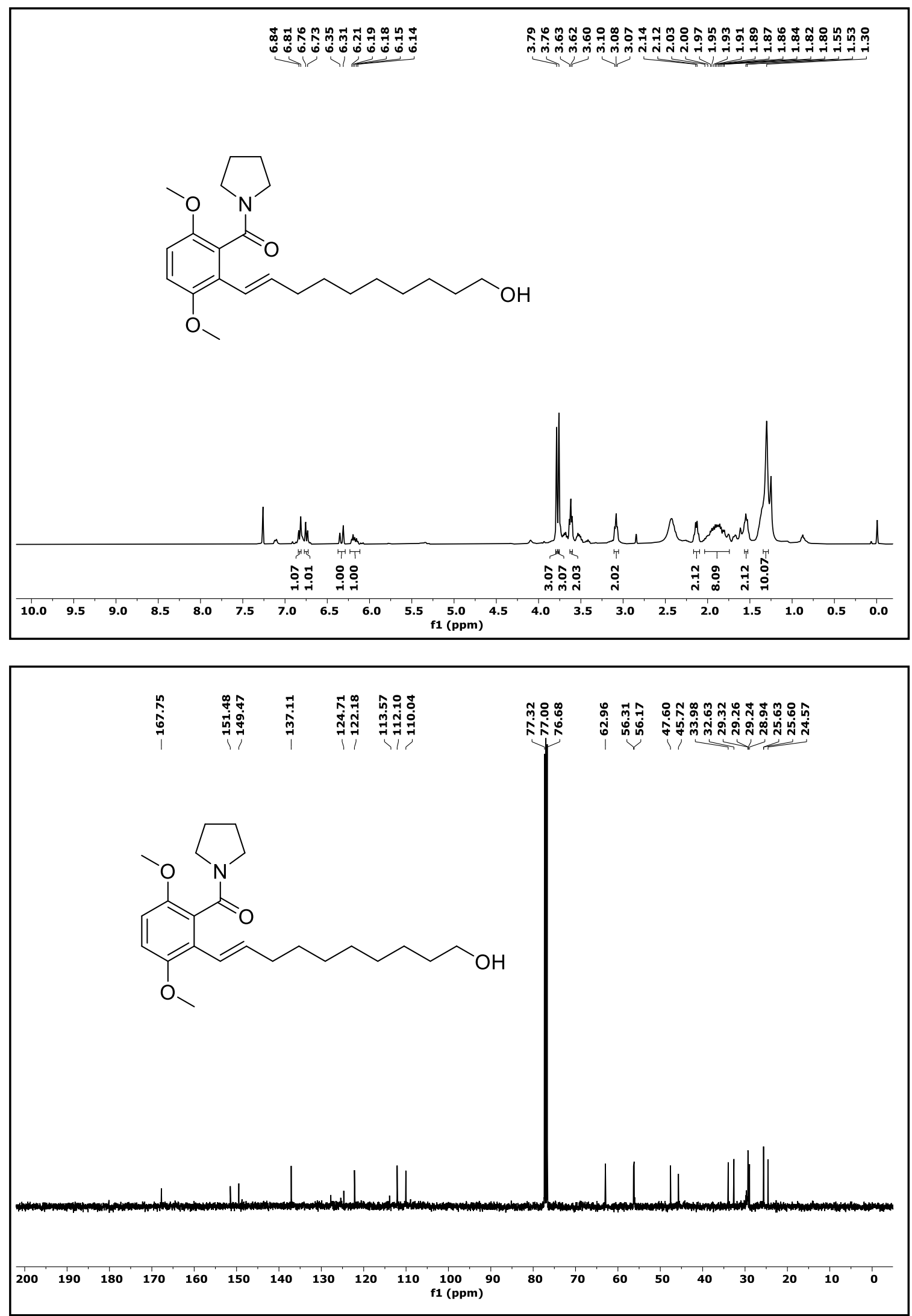
${ }^{1} \mathrm{H}$ and ${ }^{13} \mathrm{C}$ NMR Spectra of Compound 5bh. (DMSO solvent was used, $400 \mathrm{NMR} \mathrm{MHz}$ )
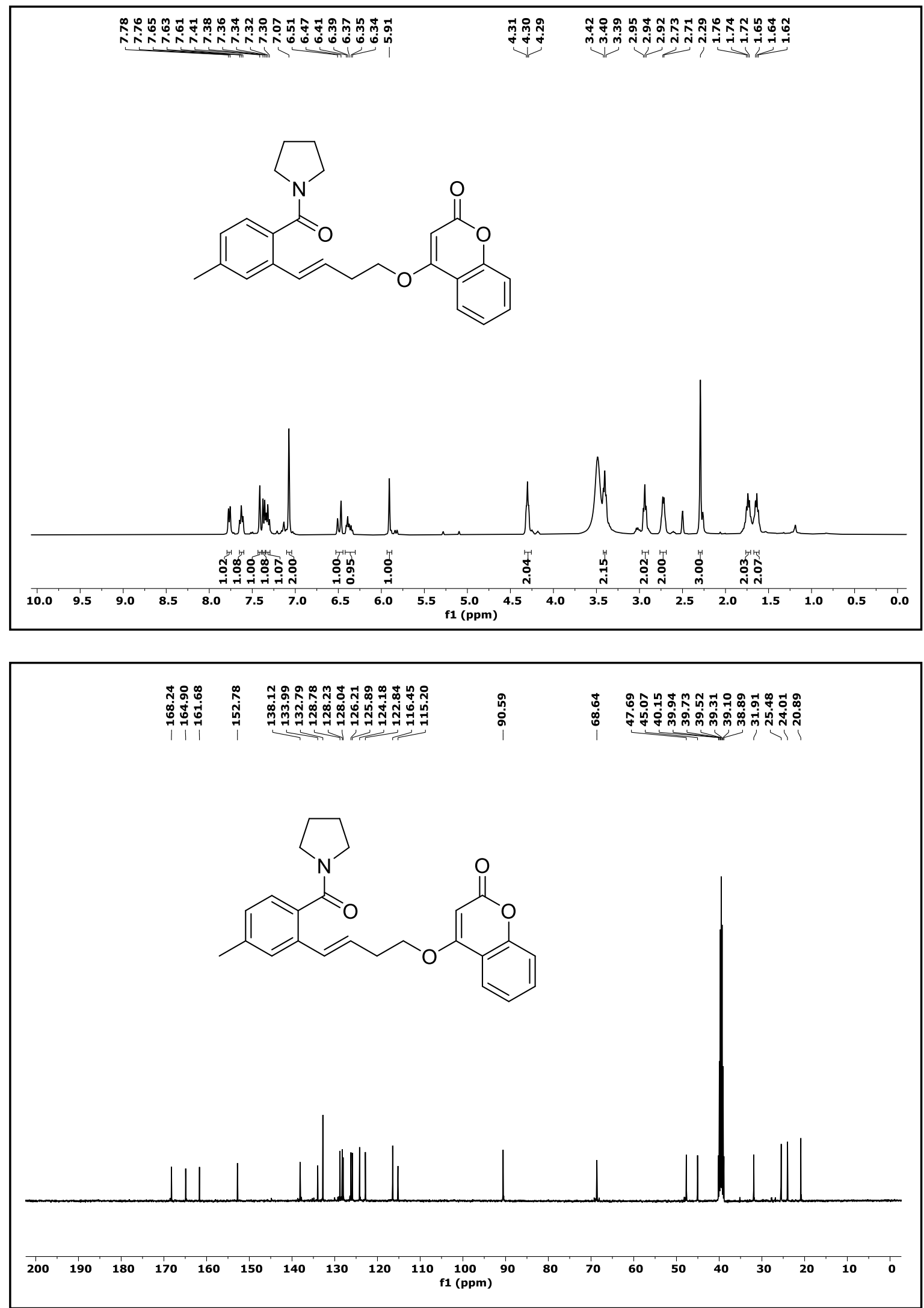
${ }^{1} \mathrm{H}$ and ${ }^{13} \mathrm{C}$ NMR Spectra of Compound 5oh. (DMSO solvent was used, $400 \mathrm{NMR} \mathrm{MHz}$ )
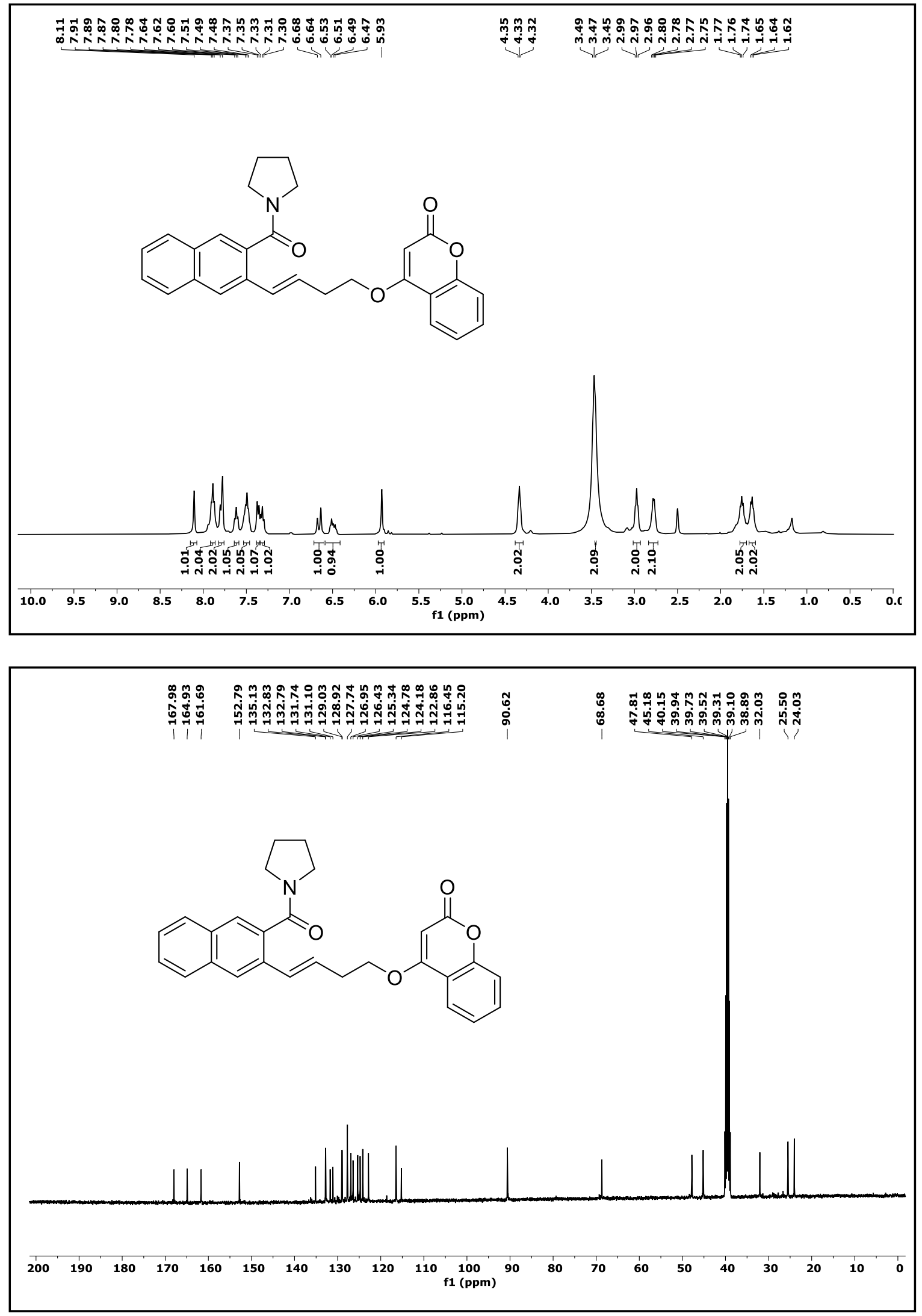
${ }^{1} \mathrm{H}$ and ${ }^{13} \mathrm{C}$ NMR Spectra of Compound 5oi. $\left(\mathrm{CDCl}_{3}\right.$ solvent was used, $\left.400 \mathrm{NMR} \mathrm{MHz}\right)$
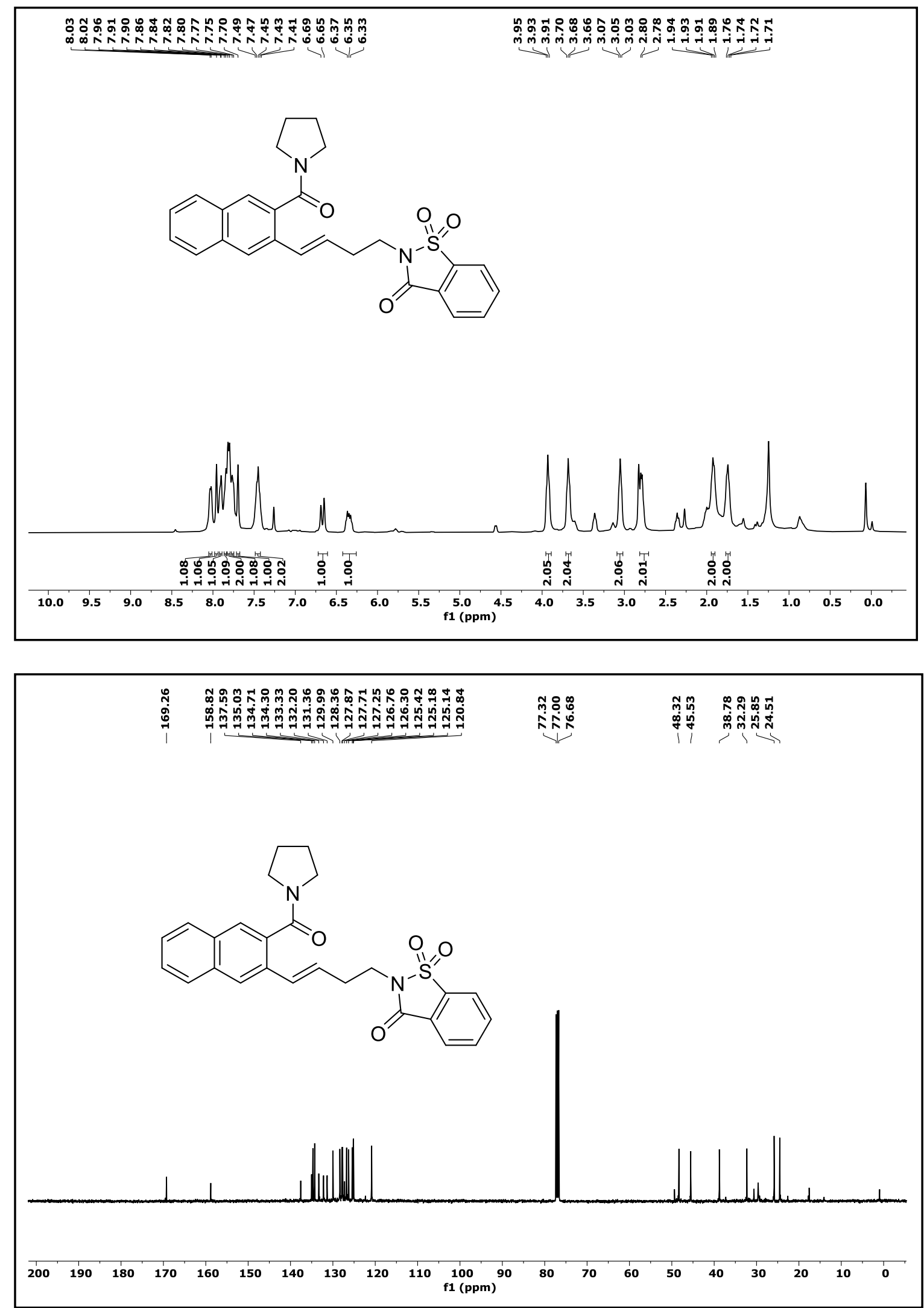
${ }^{1} \mathrm{H}$ and ${ }^{13} \mathrm{C}$ NMR Spectra of Compound 5oj/5oj'. $\left(\mathrm{CDCl}_{3}\right.$ solvent was used, $\left.400 \mathrm{NMR} \mathrm{MHz}\right)$

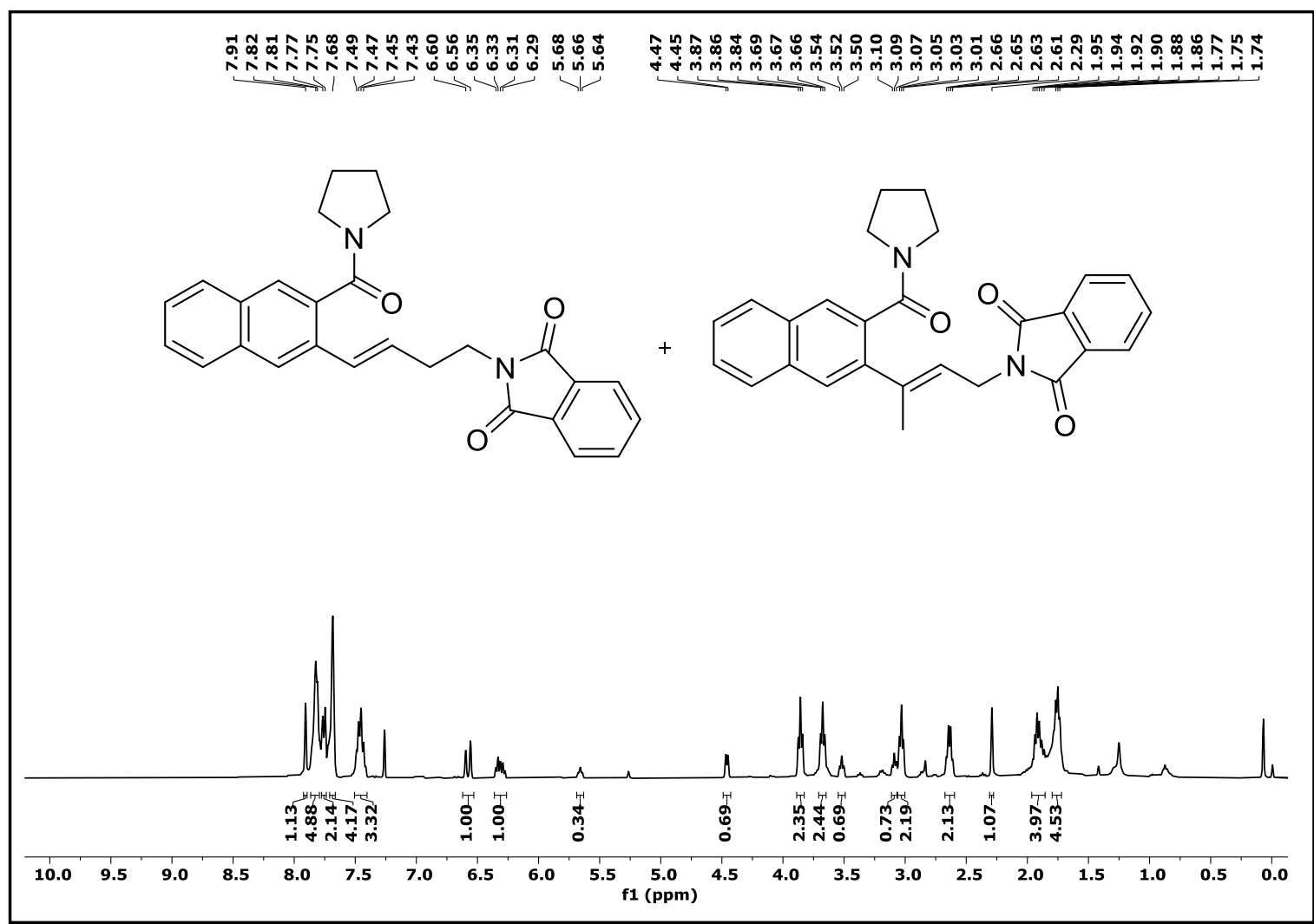

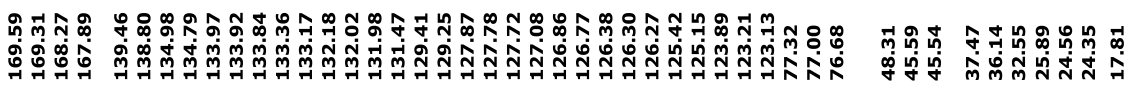<smiles>C/C(=C\CN1C(=O)c2ccccc2C1=O)c1cc2ccccc2cc1C(=O)N1CCCC1</smiles>

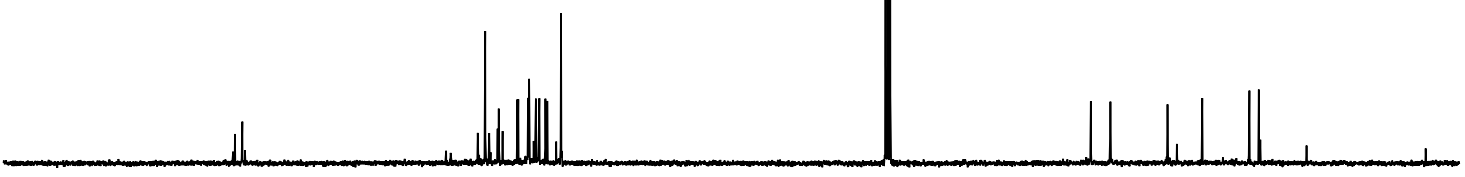

$\begin{array}{lllllllllllllllllllllll}200 & 190 & 180 & 170 & 160 & 150 & 140 & 130 & 120 & 110 & 100 & 90 & 80 & 70 & 60 & 50 & 40 & 30 & 20 & 10 & 0\end{array}$ 
${ }^{1} \mathrm{H}$ and ${ }^{13} \mathrm{C}$ NMR Spectra of Compound 5ok/5ok'. $\left(\mathrm{CDCl}_{3}\right.$ solvent was used, $\left.400 \mathrm{NMR} \mathrm{MHz}\right)$

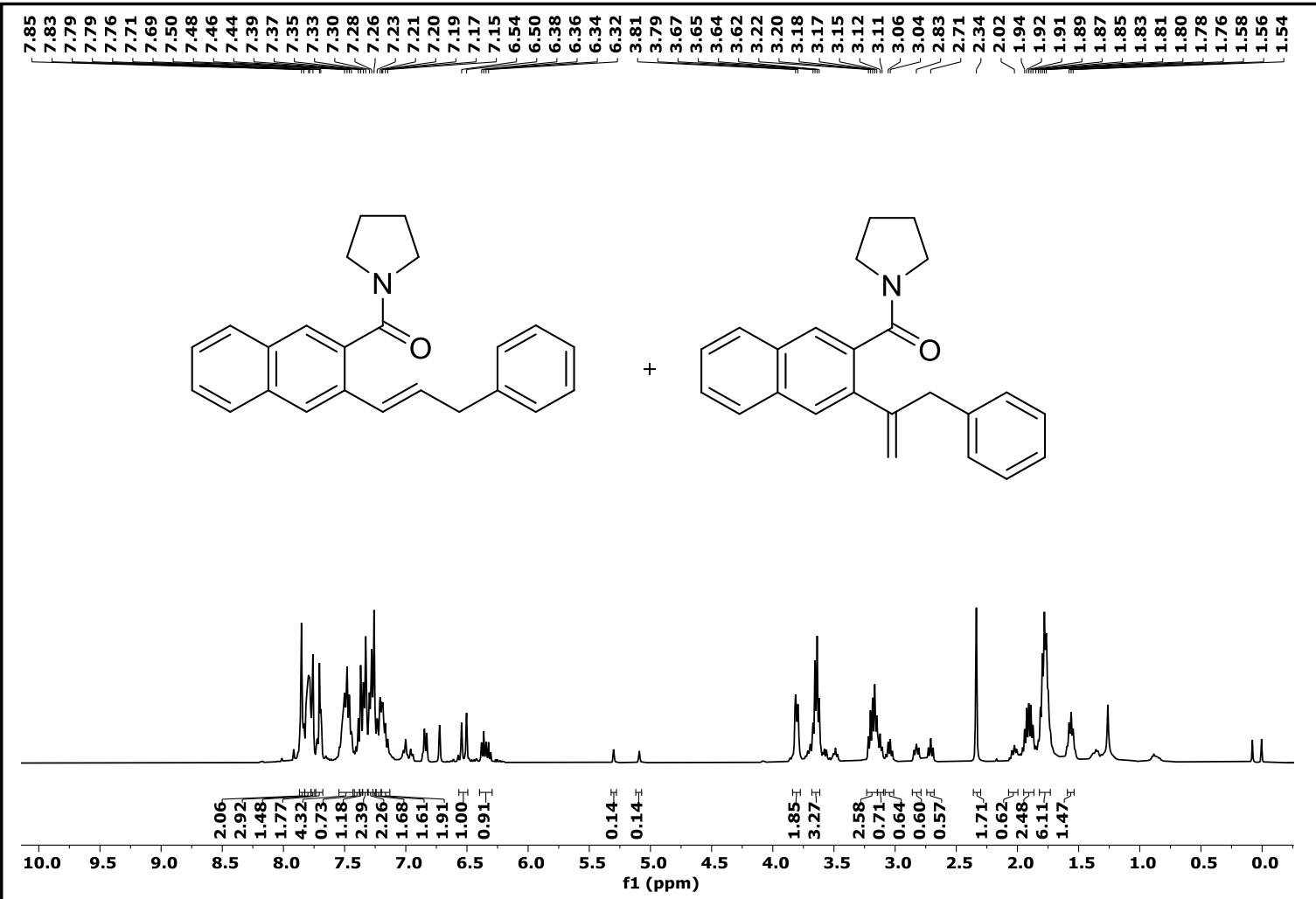

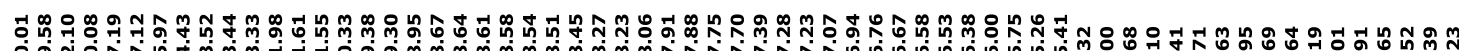

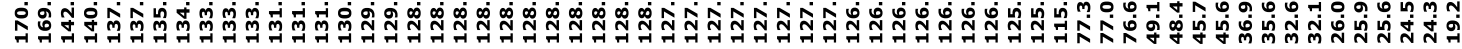<smiles>C=C(Cc1ccccc1)c1cc2ccccc2cc1C(=O)N1CCCC1</smiles>

$\begin{array}{lllllllllllllllllllll}200 & 190 & 180 & 170 & 160 & 150 & 140 & 130 & 120 & 110 & 100 & 90 & 80 & 70 & 60 & 50 & 40 & 30 & 20 & 10 & 0\end{array}$ 
${ }^{1} \mathrm{H}$ and ${ }^{13} \mathrm{C}$ NMR Spectra of Compound 6ol. $\left(\mathrm{CDCl}_{3}\right.$ solvent was used, $\left.400 \mathrm{NMR} \mathrm{MHz}\right)$
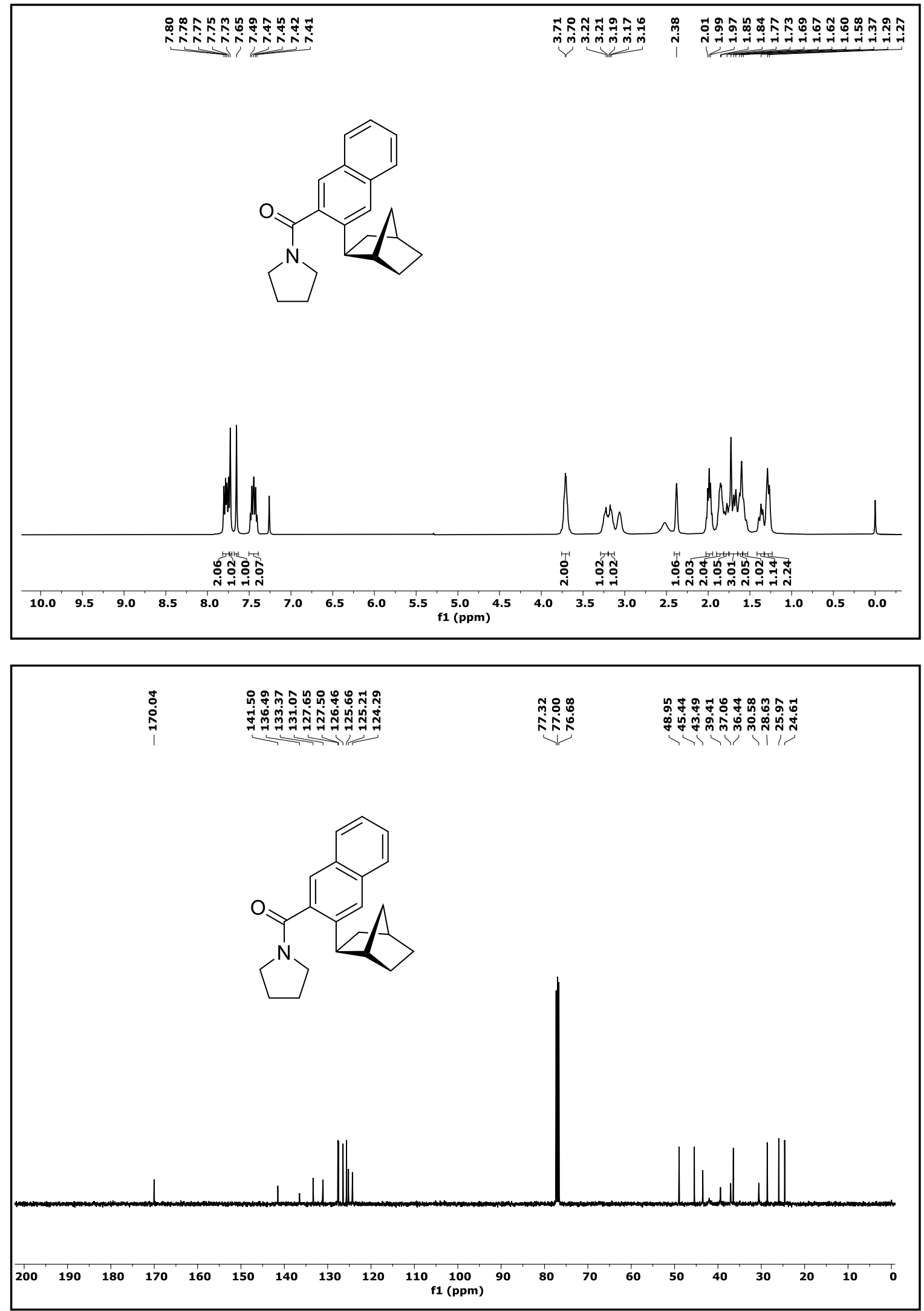
${ }^{1} \mathrm{H}$ and ${ }^{13} \mathrm{C}$ NMR Spectra of Compound 6al. $\left(\mathrm{CDCl}_{3}\right.$ solvent was used, $\left.500 \mathrm{NMR} \mathrm{MHz}\right)$

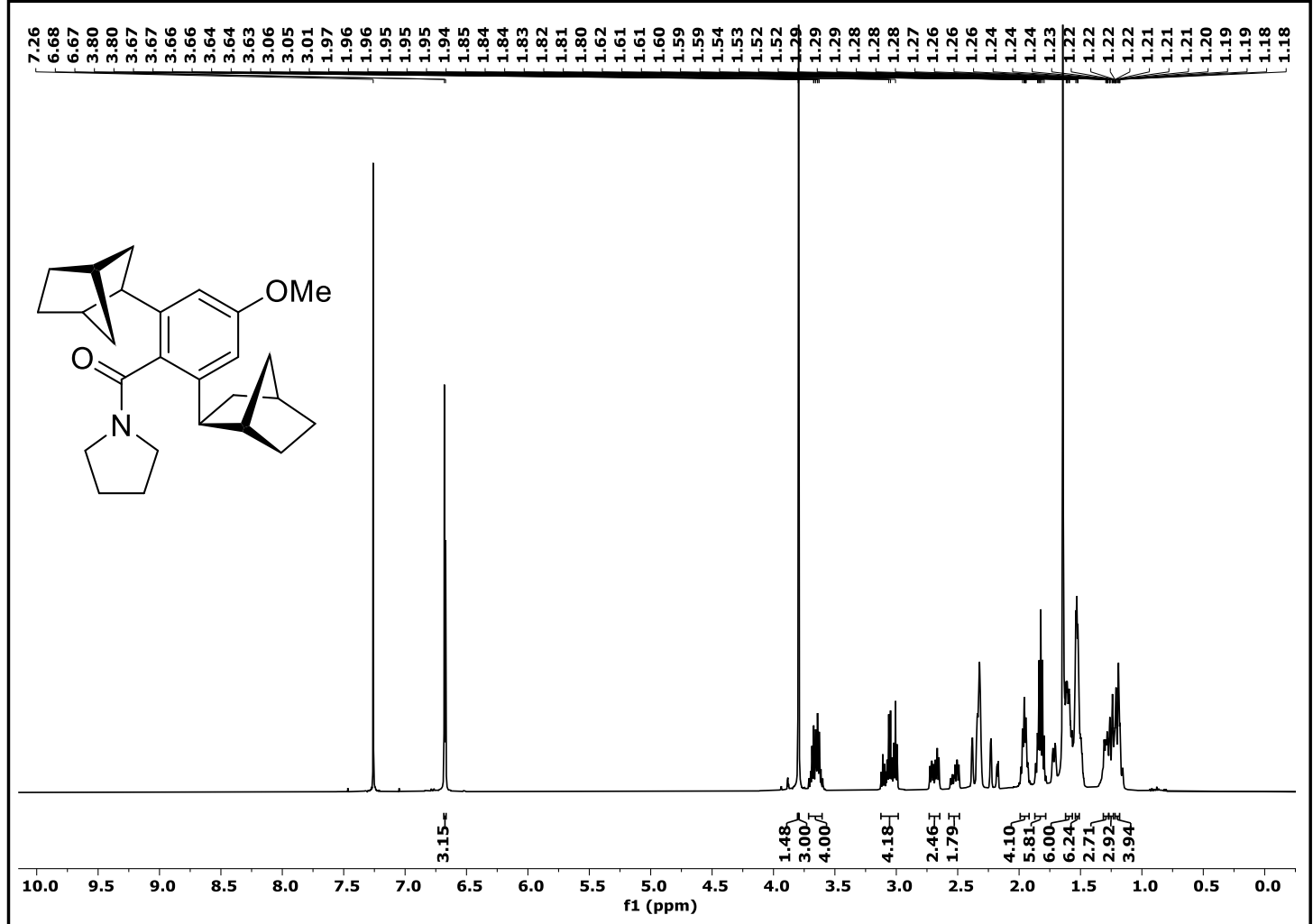

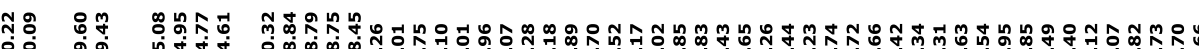

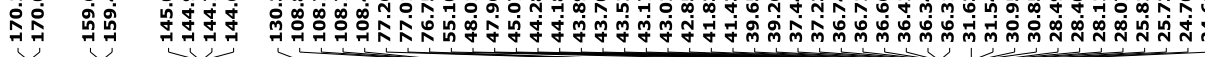

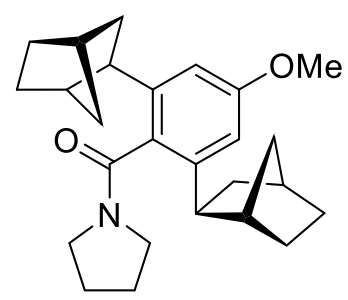

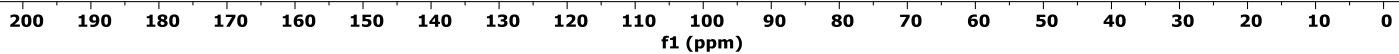


${ }^{1} \mathrm{H}$ and ${ }^{13} \mathrm{C}$ NMR Spectra of Compound 6bl. $\left(\mathrm{CDCl}_{3}\right.$ solvent was used, $\left.500 \mathrm{NMR} \mathrm{MHz}\right)$
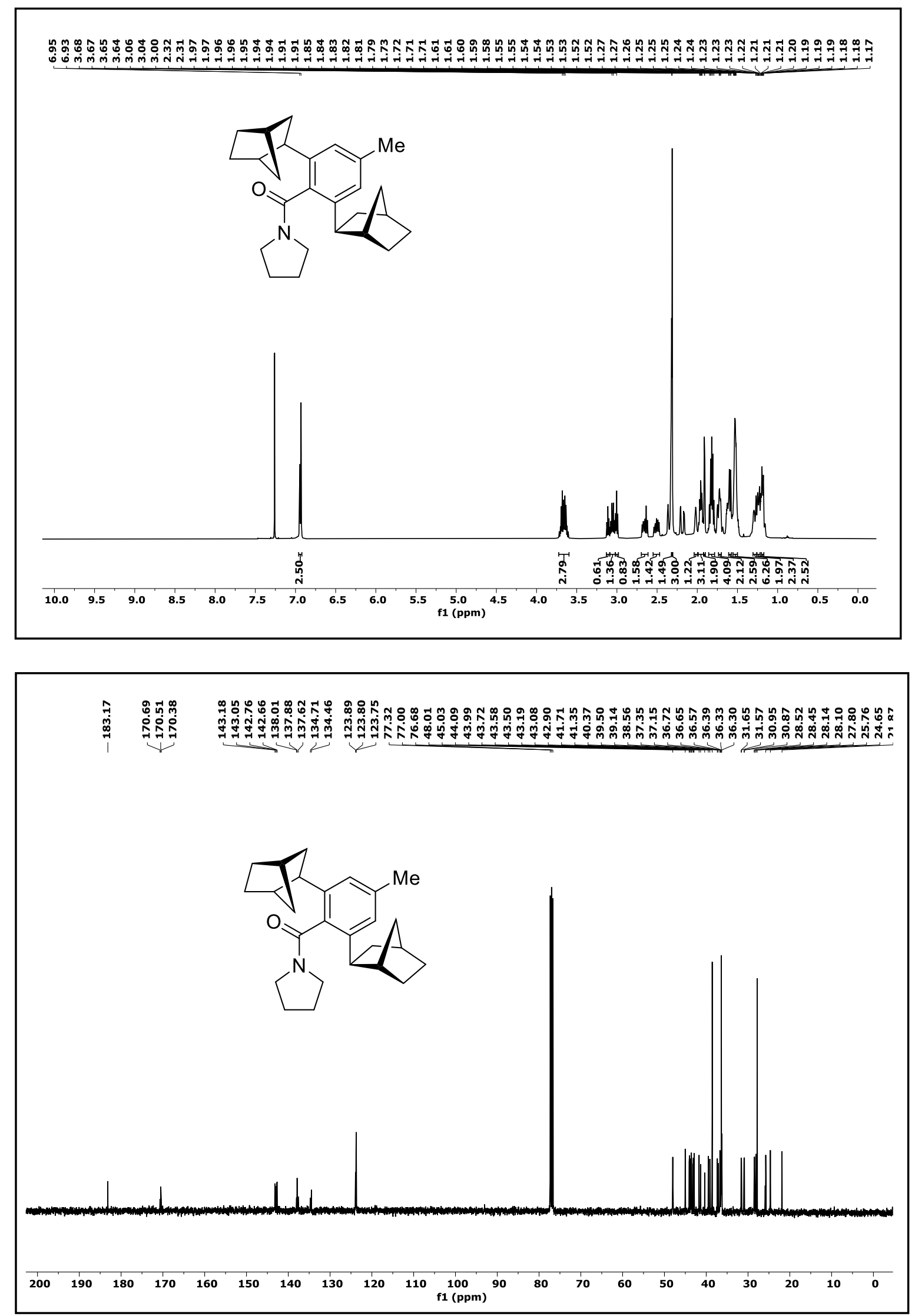
${ }^{1} \mathrm{H}$ and ${ }^{13} \mathrm{C}$ NMR Spectra of Compound $\mathbf{6 d l} .\left(\mathrm{CDCl}_{3}\right.$ solvent was used, $\left.500 \mathrm{NMR} \mathrm{MHz}\right)$
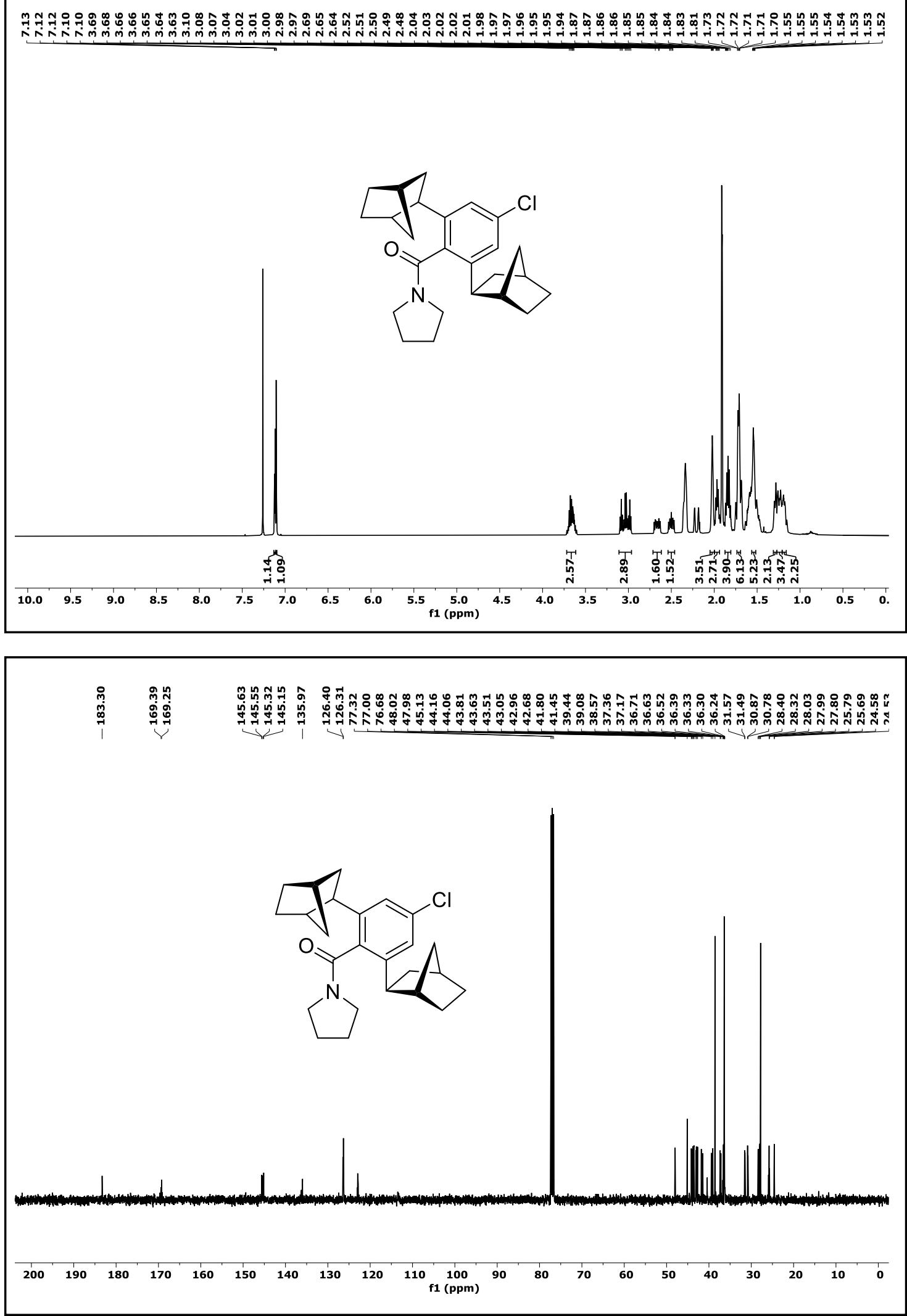
${ }^{1} \mathrm{H}$ and ${ }^{13} \mathrm{C}$ NMR Spectra of Compound 6el. $\left(\mathrm{CDCl}_{3}\right.$ solvent was used, $\left.500 \mathrm{NMR} \mathrm{MHz}\right)$

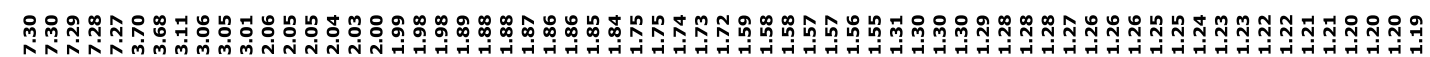<smiles>O=C(c1c(C2CC3CCC2C3)cc(Br)cc1C1CC2CCC1C2)N1CCCC1</smiles>

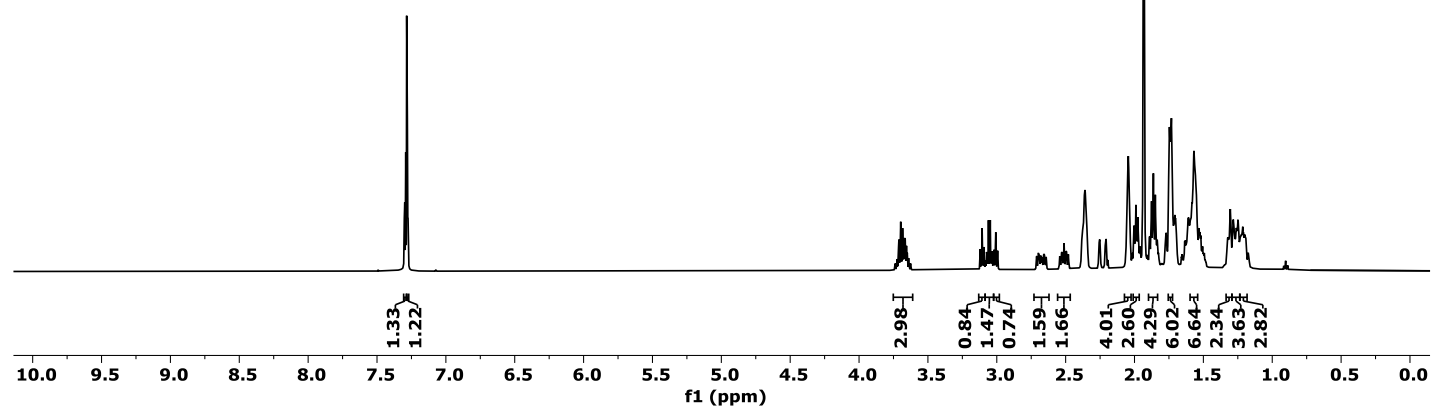

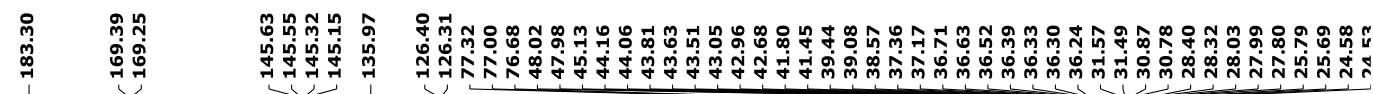

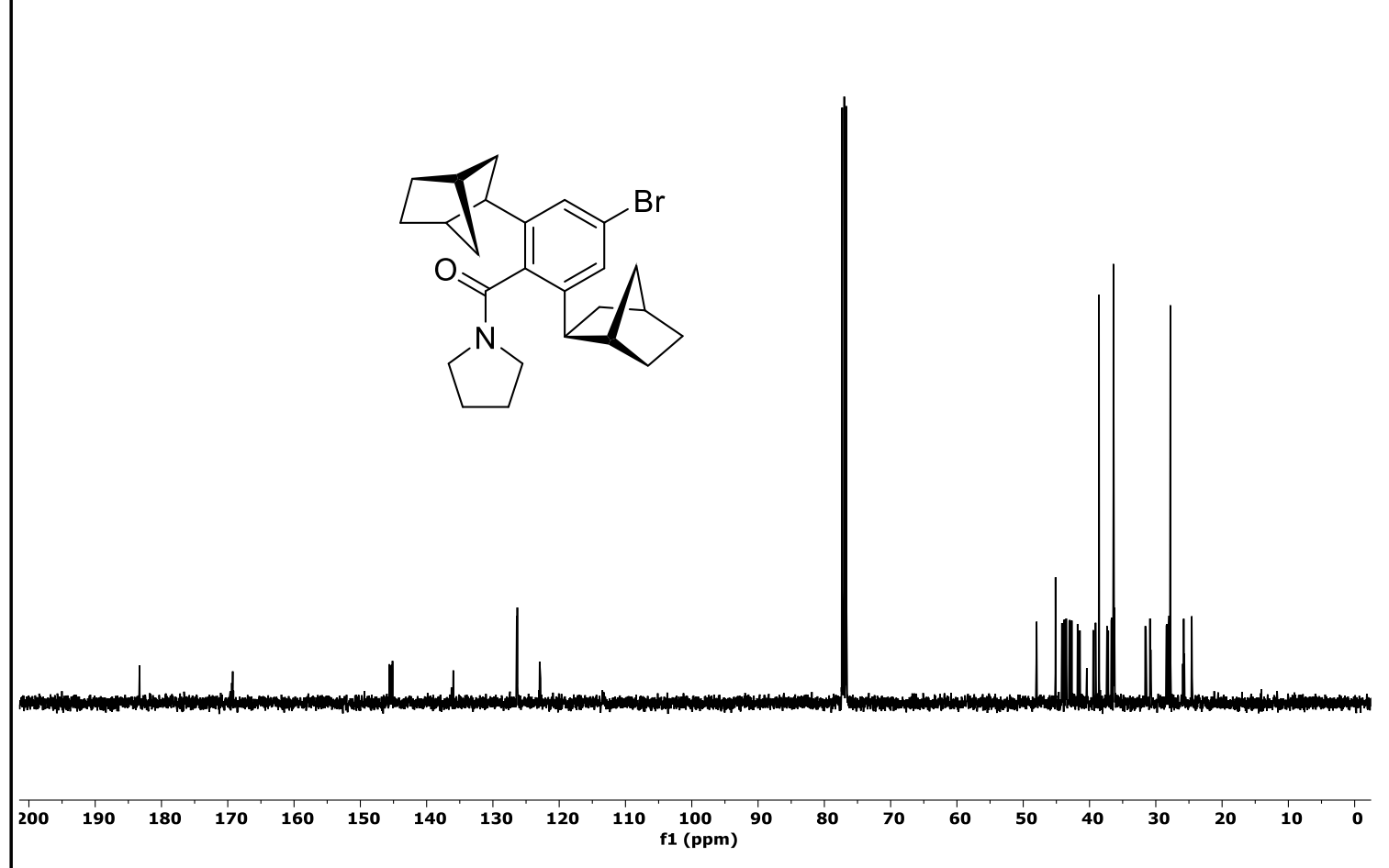

